\title{
NBSIR $73-304$
}

\section{REFERENCE-WAVEFORM GENERATION USING DEBYE DIELECTRIC DISPERSIOH}
N.S. Nahman
R.M. Jickling
D.R. Holt

Electromagnetics Division

Institute for Basic Standards

National Bureau of Standards

Boulder, Colorado 80302

December 1972

Final Report

Prepared for:

Department of Defense

Calibration Coordination Group 69-33

U. S. Army Metrology \& Calibration Center

Redstone Arsenal, Alabama 35809 

NBSIR 73-304

\section{REFERENCE-WAVEFORM GENERATION USING DEBYE DIELECTRIC DISPERSION}

N.S. Nahman

R.M. Jickling

D.R. Holt

Electromagnetics Division

Institute for Basic Standards

National Bureau of Standards

Boulder, Colorado 80302

December 1972

Final Report

Prepared for:

Department of Defense

Calibration Coordination Group 69.33

U. S. Army Metrology \& Calibration Center

Redstone Arsenal, Alabama 35809

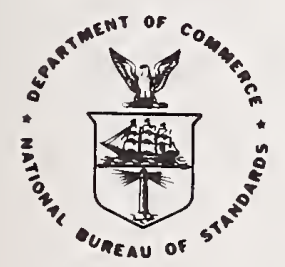

U.S. DEPARTMENT OF COMMERCE, Frederick B. Dent, Secretary 

CONTENTS

Chapter

Page

Abstract

1. INTRODUCTION

1.1 Definition of Reference-Waveform Generation 2

1. 2 Definition of Available-Waveform 2

1. 3 NBS Reference-Waveform Generator Characterization 2

1.4 Determination of the Available-Waveform 3

2. DEVELOPMENT AND CONSTRUCTION 8

2.1 Uniform Line Parameters 8

2. 2 Uniform Line Design and Parameter Characterization 11

2.3 Coaxial Connector/Adapter Design and Parameters 14

2. 4 Liquid Dielectric Preparation and Parameter Measurements

2.5 Available-Waveform Computation/Evaluation 23

3. OPERATING SYSTEM 29

3.1 Electrical Network Physical Structure 29

3.2 Waveform Data Acquisition System 30

4. SUMMARY 31

REFERENCES 32

ILLUSTRATIONS 33

APPENDIX A-1 - TIME DOMAIN REFLECTOMETRY 61

ILLUSTRATIONS FOR APPENDIX A-1 78

APPENDIX A-2 - WAVEFORM DATA FOR FIGURES 86

2. 15 and 2.16 
Figure 1.1 Reference waveform parameters.

Figure 1.2 The available waveform e $e_{a}(t)$ across a $50 \mathrm{ohm} \mathrm{load.} \quad 34$

Figure 1.3 The defining network for the available waveform $e_{a}(t) . \quad 35$

Figure 1.4 The generated waveform $e_{0}(t) . \quad 36$

Figure 1.5 (A) Squared difference of the output voltage $e_{0}(x) c o m-37$ pared to the defined available waveform $e_{a}(x)$

(B) The output voltage $e_{0}(x)$ overlayed on the defined available waveform $e_{a}(x)$

Figure 1.6 Time domain insertion method of calibration

Figure 2.1 The equivalent circuit per meter for the uniform transmission line.

Figure 2.2 TDR signature of the nominally uniform $4.65 \mathrm{~m}$ trans- 40 mission line filled with 1.0 molal solution of 2-Heptanone in normal heptane.

Figure 2.3 Dielectric support PTFE, $r_{1}=2.38 \mathrm{~mm}, r_{2}=4.76 \mathrm{~mm}, 41$ $r_{3}=9.97 \mathrm{~mm}, \mathrm{t}=3.18 \mathrm{~mm}$.

Figure 2.4 TDR signature of the nominally uniform $1.55 \mathrm{~m}$ trans42 mission line filled with 1. 14 molal solution of 2-Heptanone in normal Heptane.

Figure 2.5 Modified and NBS adapters, external view.

Figure 2.6 Modified commercial and NBS adapters, internal view. 44

Figure 2.7 NBS adapter, engineering drawing. 45

Figure 2.8 TDR signature of the connector used with the $4.65 \mathrm{~m}$ 46 line filled with 1.0-molal solution of 2-Heptanone in n-Heptane.

Figure 2.9 TDR signature of the connector used with the $1.5 \mathrm{~mm}$ 47 or $3.10 \mathrm{~m}$ line filled with 1.14-molal solution of 2-Heptanone in $\mathrm{n}$-Heptane.

Figure 2.10 Lumped circuit equivalent networks for connector/ adapters. 
Figure 2.11 Interpolation determination of the static dielectric constant parameters $\epsilon_{r}$ and a of n-Heptane.

Figure 2.12 Digitized observed driving waveform $\mathrm{e}_{\mathrm{dl}}(\mathrm{t})$.

Figure 2.13 The observed insertion voltage $e_{d 2}(t)$ overlayed on the computed insertion voltage $\overline{\mathrm{e}}_{\mathrm{d} 2}(\mathrm{t})$ for $\tau=5.52 \mathrm{ps}$.

Figure 2.14 (A) Squared difference between the observed insertion voltage $e_{d 2}(t)$ and the computed insertion voltage $\bar{e}_{d 2}(t)$. (B) The observed insertion voltage $e_{d 2}(t)$ overlayed on the computed insertion voltage $\bar{e}_{d 2}(t)$ for $\tau=3.52 \mathrm{ps}$.

Figure 2.15 The generator output voltage $e_{0}(x)$ overlayed on the defined available waveform e $e_{a}(x)$.

Figure 2.16 The squared difference of the output voltage $e_{0}(x)$ 54 compared to the defined available waveform $e_{a}(x)$ for the waveforms shown in Fig. 2. 15.

Figure 3.1 Liquid-dielectric filling system.

Figure 3.2. A view of the liquid-filled transmission lines installed on the supporting tower.

Figure 3.3 The tunnel-diode mount attached to the lower end of a liquid-filled transmission line.

Figure 3.4 The electrical waveform sampler attached to the upper 58 end of a liquid-filled transmission line.

Figure 3.5 Overflow venting.

Figure 3.6 Block diagram of the waveform data acquisition system. 


\section{ILLUSTRATIONS IN APPENDIX}

Page

Figure A-1 The input equivalent circuit for a uniform lossless transmission line terminated in an impedance $Z(s)$ and driven by a bandlimited generator $E_{g}(s) . R_{01}$ is the transmission line characteristic impedance.

Figure A-2 The bandlimited driving voltage, $e_{g}(t)$.

Figure A-3 The TDR equivalent circuit for a series inductive dis-

continuity $\mathrm{s} L$ between two uniform transmission lines of

characteristic impedances $R_{01}$ and $R_{02}$ respectively.

Figure A-4 Qualitative time domain responses for the total inductive load voltage. A delay line of time delay $t_{1} / 2$ and characteristic impedance $R_{0 I}$ is used to isolate in time the observation point and the discontinuity.

Figure A-5 The input equivalent circuit for a uniform lossless transmission line termination in an admittance $\mathrm{Y}(\mathrm{s})$ and driven by a bandlimited generator $I_{g}(s) \cdot G_{01}$ is the transmission line characteristic admittance.

Figure A-6 The bandlimited driving current, $i_{g}(t)$.

Figure A-7 The TDR equivalent circuit for a shunt capacitive discontinuity $\mathrm{sC}$ at the junction of two uniform transmission lines of characteristic admittances $G_{01}$ and $G_{02}$, respectively.

Figure A-8 Qualitative time domain responses for the total capacitive load voltage. 


\section{LIST OF TABLES}

Table Number

Page

Table 2.1 Transmission Line Parameters

Table 2.2 Connector Parameters

Table 2.3

Relative $1 \mathrm{kHz}$ Dielectric Constants of Polar

Solutions and Nonpolar Solvents as $23^{\circ} \mathrm{C}$

Table 2.4 Summary of Electrical Characteristics for

$4.65 \mathrm{~m}$ Reference Waveform Generator 
REFERENCE-WAVEFORM GENERATION USING DEBYE DIELECTRIC DISPERSION

by

N. S. Nahman, R. M. Jickling and D. R. Holt

\section{ABSTRACT}

This report discusses the theory, construction, and operation of Reference-Waveform Generators using a tunnel diode transition-waveform generator driving 4.65 meter ( 15 foot), 3. 10 meter ( 10 foot), and 1.55 meter ( 5 foot) liquid-dielectric uniform lossy coaxial lines to produce known 0.2 volt transition waveforms across $50 \mathrm{ohms}$ with (10-90\%) transition times from 205 to 560 picosecond. Each resultant Available-Waveform is characterized in terms of its departure from the step response of the uniform lossy coaxial line operating into a 50 ohm load. The liquid dielectric solutions are dispersive with relaxation times of the order of 4 picoseconds in which heptane is the solvent and the ketones, butanone, heptanone and octanone are the solutes in concentrations ranging from 0.25 - to 2-molal.

Key words: Pulse distortion in transmission lines; pulse techniques; reference waveform generation; time domain measurements; transient response Debye dielectric. 


\section{INTRODUCTION}

1.1 Definition of Reference-Waveform Generation

Reference-Waveform Generation is the production of an electrical waveform of known mathematical form with a known source impedance.* A Reference-Waveform Generator may be completely characterized in terms of its open-circuit voltage in the time domain $e_{g}(t)$, source impedance (in the frequency domain) $Z_{g}(s)$ and a time window (interval) over which e $g(t)$ and $Z_{g}(s)$ are valid, Fig. 1. 1 [ 1].

\subsection{Definition of Available-Waveform}

A Reference-Waveform Generator may be characterized by its Available-Waveform e $e_{a}(t)$. The Available-Waveform is defined as the voltage $e_{a}(t)$ produced by the Reference-Waveform Generator across a $50 \mathrm{ohm}$ load, Fig. 1.2. In practice the Available-Waveform is a well defined mathematical expression.

\subsection{NBS Reference-Waveform Generator Characterization}

For the NBS Reference-Waveform Generators the mathematical form and source impedance of the generated waveform is primarily determined by the bandwidth limitation produced by a lossy uniform coaxial transmission line. The transmission line los is produced by filling the line with a liquid polar dielectric which introduces Debye dispersion.

The Available-Waveform of the NBS generator is produced as the step response of the lossy transmission line terminated in $50 \mathrm{ohms}$ and driven by a $50 \mathrm{ohm}$ source, Fig. 1.3. In practice, the actual GeneratedWaveform e $e_{0}(t)$ does depart from the defined value; this departure can be characterized and is the fundamental error in generating the known waveform.

* Note that the objective here is to generate a waveform of defined mathematical form; the objective is not to generate the shortest possible transition (rise) time step-like waveform of unknown shape. 
Each Generated-Waveform $e_{0}(t)$ is produced by drixing a length of lossy transmission line with a $50 \mathrm{ohm}$, ultra-fast tunnel-diode transition waveform generator. Connectors at each end couple the line to the tunnel-diode generator and to the $50 \mathrm{ohm}$ load, Fig. 1.4. Imperfections in the nominal $50 \mathrm{ohm}$ terminations are considered negligible, and the two connectors are assumed identical. Therefore, the Generated-Waveform $e_{o}(t)$ does depart from the defined Available-Waveform $e_{a}(t)$. The departures are directly traceable to the three factors: (1) the physical impossibility of generating a perfect step function as the driving function $e_{1}(t)$, Figure 1.4, (2) the presence of non-uniform connectors (M networks Figure 1.4) at the ends of the uniform liquid filled transmission line, and (3) our inability to separate the driving waveform $e_{1}(t)$ from that of the observed system response used to characterize the driving waveform. The departure may be characterized by the squared error $\Delta^{2}(t)$ between the defined Available-Waveform $e_{a}(t)$ and the GeneratedWaveform e $e_{0}(t)$, Fig. 1.5.

1.4 Determination of the Available-Waveform

The Available-Waveform is calibrated by the insertion method shown in Fig. 1.6; the voltages and parameters are defined as follows:

$$
\begin{aligned}
e_{d l}(t) \equiv & \text { The recorded sampling-oscillo- } \\
& \text { scope analog output voltage when } \\
& \text { the } 50 \text { ohm transition generator } \\
& e_{1}(t) \text { is connected directly to the } \\
& 50 \text { ohm sampling head. } \\
e_{d 2}(t) \equiv & \text { The recorded sampling-oscillo- } \\
& \text { scope analog output when the } \\
& \text { lossy transmission line with the } \\
& M \text { connectors is inserted between }
\end{aligned}
$$


the transition generator and the sampling os cillos cope.

$$
\begin{aligned}
& Z_{2}(s), Z_{3}(s), Z_{4}(s) \equiv \text { Lumped impedance parameters } \\
& \text { characterizing an } \mathrm{M} \text {-connector.* } \\
& \mathrm{N}(\mathrm{s}) \equiv \text { The input port to analog-output } \\
& \text { transfer function for a sampling } \\
& \text { oscilloscope having a } 50 \mathrm{ohm} \\
& \text { input port impedance. } \\
& \gamma(\mathrm{s}), Z_{0}(s), l \equiv \text { The propagation function, charac- } \\
& \text { teristic impedance, and length, } \\
& \text { respectively, of the lossy uniform } \\
& \text { transmission line. }
\end{aligned}
$$

In terms of the defined parameters, the frequency domain voltages corresponding to $e_{d 1}(t)$ and $e_{d 2}(t)$ are given by

$E_{d 1}(s)=\mathcal{E}\left[e_{d 1}(t)\right]=\frac{E_{1}(s)}{2} N$

$E_{d 2}(s)=\mathcal{L}\left[e_{d 2}(t)\right]$

$$
=E_{1}(s) N \frac{50 Z_{0} e^{-l \gamma}}{\left\{\left[50\left(1+Z_{3} / Z_{2}\right)+Z_{3}\right]\left(1+z_{0} / Z_{4}\right)+\left(1+50 / Z_{2}\right) Z_{0}\right\}^{2}}
$$

where $Z_{0}, Z_{2}, Z_{3}, Z_{4}, N$, and $\gamma$ are functions of $s . \mathcal{L}^{-1}\left[E_{d 2}(s)\right]$ is the initial (direct) waveform appearing at the oscilloscope output terminals and is valid for the time interval $0<t<3 \mathrm{~T}$ where

* Although a connector is a distributed network, it may be considered as a networl of lumped impedances if its transmission time is small compared to the transition time of a pulse passing through it. 


$$
T=\lim _{\omega \rightarrow \infty}\left\{\frac{\text { Imaginary Part of } \ell \gamma(j \omega)}{\omega}\right\}
$$

This assumes that the transmission time through the connectors is negligible in comparison to T. From (1.1) we have

$$
E_{1}(s) N=2 E_{d l}(s)
$$

which when put into (1.2) obtains the output voltage $E_{\mathrm{d} 2}(\mathrm{~s})$ in terms of the observable voltage $E_{\mathrm{dl}}(\mathrm{s})$,

$E_{d 2}(s)=2 E_{d l}(s) \frac{50 Z_{o} e^{-l \gamma}}{\left\{\left[50\left(1+Z_{3} / Z_{2}\right)+Z_{3}\right]\left(1+Z_{o} / Z_{4}\right)+\left(1+50 / Z_{2}\right) Z_{o}\right\}^{2}}$

and

$$
\begin{gathered}
e_{d 2}(t)=\mathcal{L}^{-1} 2 E_{d 1}(s) \frac{50 z_{0} e^{-l \gamma}}{\left\{\left[50\left(1+z_{3} / z_{2}\right)+z_{3}\right]\left(1+z_{0} / z_{4}\right)+\left(1+50 / z_{2}\right) z_{0}\right\}^{2}} \\
0<t<3 T
\end{gathered}
$$

Equation (1.6) provides the basis for calibrating the Generated Waveform. The calibration procedure consists of comparing the experimentally observed generated-waveform $e_{d 2}(t)$ with a computed value $\overline{\mathrm{e}_{\mathrm{d} z}(t)}$ from (1.6). The quantities within (1.6) are determined by independent experiments; consequently, agreement between the observed $e_{d 2}(t)$ and the computed waveform establishes that the transmission line/connector voltage transfer function

$G(s)=\frac{E_{d 2}(s)}{E_{d l}(s)}=\frac{100 Z_{0} e^{-l \gamma}}{\left\{\left[50\left(1+Z_{3} / Z_{2}\right)+Z_{3}\right]\left(1+Z_{0} / Z_{4}\right)+\left(1+50 / Z_{2}\right) Z_{0}\right\}^{2}}$ 
is valid. It is then possible to consider the departure between the Generated-Waveform

$$
e_{0}(t)=\mathcal{L}^{-1}\left[E_{1}(s) G(s)\right], \quad 0<t<3 T
$$

and the observed-waveform $e_{d 2}(t)$

$$
\mathrm{e}_{\mathrm{d} 2}(\mathrm{t})=\mathcal{L}^{-1}\left[\mathrm{E}_{\mathrm{d} 1}(\mathrm{~s}) \mathrm{G}(\mathrm{s})\right], \quad 0<\mathrm{t}<3 \mathrm{~T} .
$$

Clearly, the departure between (1.8) and (1.9) is caused by $E_{1}(s)$ being different from $E_{d l}(s)$ where $E_{l}(s)$ and $E_{d l}(s)$ are related by

$$
E_{1}(s)=\frac{2 E_{d 1}(s)}{N(s)} \text {, }
$$

that is, the oscilloscope used to observe $e_{1}(t)$ transforms (distorts) $e_{1}(t)$ into $e_{d l}(t)$. Consequently, due to the uncertainty with which $e_{1}(t)$ is known, $e_{o}(t)$ is bounded by the time domain response (1.9). Because the Reference-Waveform Generator is employed without the specific oscilloscope used for calibration, all of the uncertainty due to the oscilloscope, $N(s)$, has to be transferred to $E_{1}(s)$, that is, (1.9) is used in place of $(1.8)$ in computing the uncertainty in the Available-Waveform.

It is then possible to compare the Generated-Waveform $e_{0}(t)$ with the defined Available-Waveform

$$
\mathrm{e}_{\mathrm{a}}(\mathrm{t})=\mathcal{L}^{-1}\left\{\frac{1}{\mathrm{~s}} \frac{50 \mathrm{z}_{\mathrm{o}} \mathrm{e}^{-\ell \gamma}}{\left(50+\mathrm{z}_{\mathrm{o}}\right)^{2}}\right\} ; \quad 0<\mathrm{t}<3 \mathrm{~T}
$$

which is the step response of the lossy transmission line driven from a $50 \mathrm{ohm}$ source and terminated in $50 \mathrm{ohms}$. 
$e_{0}(t)$ and $e_{a}(t)$ are compared in terms of the squareddifference between them

$$
\Delta^{2}(x)=\left[e_{a}(x)-e_{d 2}(x)\right]^{2}
$$

Note that $e_{\mathrm{d} 2}(t)$ is used in place of $e_{0}(t)$ so that the uncertainty due to the oscilloscope is transferred to the driving generator $e_{1}(t)$. The independent variable $x$ is a relative time variable. $\Delta^{2}(x)$ is minimized by superimposing the waveforms $e_{a}(x)$ and $e_{d 2}(x)$ upon each other and aligning the waveforms by $x$-translation so that (1.12) is minimized in some meaningful way. For example, one choice could be to minimize the quantity,

$$
\varphi=\frac{1}{2 T} \int_{T}^{3 T} \Delta^{2}(x) d
$$




\section{DEVELOPMENT AND CONSTRUCTION}

A uniform transmission line filled with a (lossy) polar-liquid dielectric and physically terminated in connectors forms an integral part of the reference waveform generator. Under the current project, $4.65 \mathrm{~m}$ (15 ft. ), $3.10 \mathrm{~m}(10 \mathrm{ft.})$ and $1.55 \mathrm{~m}$ ( $5 \mathrm{ft}$.$) lines have been con-$ structed consisting of rigid sections of coaxial transmission line mounted in a vertical position to simplify filling and draining of the liquid dielectric through special coaxial connectors/adapters.

\section{1 Uniform Line Parameters}

The uniform transmission line is primarily bandwidth limited by Debye dielectric loss and to a lesser degree by planar skin effect loss. The transmission line equivalent circuit per meter is shown in Fig. 2. 1. The impedance per meter and the admittance per meter are given by $Z(s)$ and $Y(s)$, respectively, [1],

$$
\begin{aligned}
& Z(s)=s L+K \sqrt{s}, \text { ohms } / m \\
& Y(s)=s C_{1}+\frac{s C_{2}}{1+s R C_{2}} \text {, mhos } / m
\end{aligned}
$$

$\mathrm{L}$ and $\mathrm{K}$ are respectively the external inductance (henrys/m) and the planar skin effect coefficient (ohms/[m(radians/sec) $\left.\left.{ }^{1 / 2}\right]\right) . C_{1}$ is the high frequency limit capacitance,

$$
C_{1}=\lim _{|s| \rightarrow \infty} \frac{Y(s)}{s}, \quad \text { farads } / m
$$

While $C_{1}+C_{2}$ is the low frequency limit capacitance

$$
C_{1}+C_{2}=\lim _{|s| \rightarrow 0} \frac{Y(s)}{s}, \text { farads } / m
$$


Also, the time constant $\mathrm{RC}_{2}$ is defined as the Debye relaxation time, $\tau$, that is

$$
\tau=\mathrm{RC}_{2}
$$

The parameters $\mathrm{L}, \mathrm{K}, \mathrm{C}_{1}$ and $\mathrm{C}_{1}+\mathrm{C}_{2}$ can be computed from the transmission line physical dimensions and material parameters $\mu, \sigma$, and $\epsilon(s)$ as shown in [1].

The characteristic impedance $Z_{0}(s)$ and the propagation function $\gamma(\mathrm{s})$ are given by

$$
\begin{aligned}
Z_{0}(s) & =\sqrt{Z(s) / Y(s)} \\
& =\sqrt{(s L+K \sqrt{s})\left[\mathrm{sC}_{1}+\frac{\mathrm{sC}_{2}}{1+s \tau}\right]^{-1}}
\end{aligned}
$$

and

$$
\begin{aligned}
\gamma(s) & =\sqrt{Z(s) Y(s)} \\
& =\sqrt{(s L+K \sqrt{s})\left[s_{1}+\frac{s C_{2}}{1+s \tau}\right]}
\end{aligned}
$$

The admittance/m, $Y(s)$, can also be expressed in the form $A \in(s)$ where $A$ is a constant and $\epsilon(s)$ the complex dielectric function (see Section 2.4). At low frequencies $|\mathrm{K} \sqrt{\mathrm{s}}|$ is negligible compared to $|\mathrm{sL}|$. Setting $K \sqrt{\mathrm{s}}=0$ and taking the limit of (2.6) and (2.7) as $|s| \rightarrow 0$, the low frequency limits for the characteristic impedance and the propagation function a re obtained 


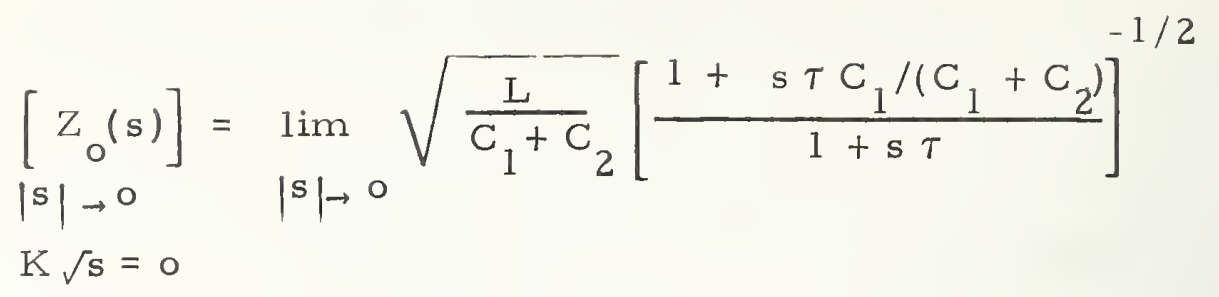

$$
\begin{aligned}
& =\sqrt{\frac{L}{C_{1}+C_{2}}} \\
& \lim _{|s| \rightarrow 0} s \sqrt{L\left(C_{1}+C_{2}\right)}\left[\frac{1+s \tau C_{1} /\left(C_{1}+C_{2}\right)}{1+s \tau}\right]^{-1 / 2} \\
& K \sqrt{s}=0 \\
& =s \sqrt{L\left(C_{1}+C_{2}\right)}
\end{aligned}
$$

and

In the high frequency limit, (2.6) and (2.7) yield

$$
\begin{aligned}
{\left[\begin{array}{c}
Z_{0}(s) \\
|s| \rightarrow \infty
\end{array}\right.} & =\lim _{|s| \rightarrow \infty} \sqrt{\frac{L}{C_{1}}} \sqrt{\left(1+\frac{K / L}{\sqrt{s}}\right)\left(1+\frac{C_{2} / C_{1}}{1+s \tau}\right)^{-1}} \\
& =\sqrt{\frac{L}{C_{1}}}
\end{aligned}
$$

and

$$
\begin{aligned}
{\left[\begin{array}{l}
\gamma(s)] \\
|s| \rightarrow \infty
\end{array}\right.} & =\lim _{|s| \rightarrow \infty} s \sqrt{L_{1}} \sqrt{\left(1+\frac{K / L}{\sqrt{s}}\right)\left(1+\frac{C_{2} C_{1}}{1+s \tau}\right)} \\
& =s \sqrt{L_{1}}
\end{aligned}
$$


By virtue of the limiting values (2.8) and (2.10), it can be seen that as the frequency is increased, the characteristic impedance magnitude $\left|Z_{0}(s)\right|$ increases from $\sqrt{L /\left(C_{1}+C_{2}\right)}$ at low frequencies to $\sqrt{\mathrm{L} / \mathrm{C}_{1}}$ at high frequencies. Also, from (2.9) and (2.11) it is seen that as the frequency is increased, the time delay decreases from $\sqrt{L\left(C_{1}+C_{2}\right)}$ at low frequencies to $\sqrt{L_{1}}$ at high frequencies.

\section{2 Uniform Line Design and Parameter Characterization}

Commercial $50 \mathrm{ohm}$ coaxial transmission lines in $4.65 \mathrm{~m}$ (15 ft.), $3.10 \mathrm{~m}$ (10 ft.) and $1.55 \mathrm{~m}$ ( $5 \mathrm{ft}$. ) lengths of $2.22 \mathrm{~cm}(7 / 8$-inch) diameter rigid copper with EIA standard flanges have been employed. The 4.65 meter commercial line was directly filled with the liquid for the first design. Consequently, the resultant (filled) characteristic impedance was approximately $33 \mathrm{ohms}$. Subsequent designs for the $3.10 \mathrm{~m}$ and $1.5 \mathrm{~m}$ lines replaced the original $0.866 \mathrm{~cm}$ ( 0.341 inch) diameter center conductor in the commercial lines with a smaller diameter conductor (0.476 cm, $3 / 16$ inch). This size for the new inner conductors was selected from easily available stock tubing such that the low-frequency characteristic impedance of the coaxial lines is approximately 50 ohms when filled with a 2 -molal solution of heptanone in heptane $\left(\epsilon_{r} \approx 3\right)$.

The initial transmission line system design $(4.65 \mathrm{~m})$ had three objectives:

1. A free-draining coaxial transmission line assembly.

2. A connector as sembly providing a means for filling and emptying the transmission line as sembly.

3. Minimization of the time for the design and development of the transmission lines/connector 
system in deference to the design of peripheral equipment for filling and emptying the trans mission line.

The unmodified commercial transmission line satisfied the first and third objectives. The second objective was met by a slight modification of a commercial connector to eliminate liquid-trapping (see Section 2. 2 for connector design).

The uniformity of the $4.65 \mathrm{~m}$ transmission line is illustrated by the time domain reflectometer (TDR) signature of the line filled with a 1. 0-molal solution of 2-heptanone in normal heptane, Fig. 2.2. Inspection of the TDR signature shows that the relative reflected voltage is -0.2 which corresponds to a characteristic impedance of 32.5 ohms referenced to $50 \mathrm{ohms}$. Static $(1 \mathrm{KHz})$ measurements of the line capacitance for a 1.0-molal concentration yield a low frequency relative dielectric constant $\epsilon_{r}(0)$ of 2.46 resulting in a low frequency characteristic impedance $Z_{0}(0)$ of 32 ohms,

$$
\begin{aligned}
& Z_{0}(s)=\sqrt{\frac{Z(s)}{Y(s)}} \\
& {\left[Z_{0}(0)\right]=\sqrt{\frac{L}{C_{1}+C_{2}}}=\sqrt{\frac{1.67 \times 10^{-7}}{1.63 \times 10^{-12}}}=32 \Omega}
\end{aligned}
$$

Also, the presence of periodic inductive reflections are clearly shown due to the solid dielectric ( $\mathrm{TFE}, \epsilon_{\mathrm{r}}=2.05$ ) pins supporting the center conductor. These reflections correspond to periodic inductive elements each being 0.175 nanohenrys spaced 27.5 centimeters apart. The time separation of the reflections is 2.88 nanoseconds which corresponds to the physical separation d between the dielectric pins of $27.5 \mathrm{~cm}$; that is, 


$$
\mathrm{d}=\frac{\mathrm{t} \nu}{2}=\frac{2.88 \times 10^{-9} \times 3 \times 10^{10}}{2 \sqrt{\epsilon_{\mathrm{r}}(0)}}=27.5 \mathrm{~cm} .
$$

Such inductive reflections may be minimized by using a smaller inner conductor and then choosing the solution concentration so that the resultant low frequency liquid dielectric constant $\epsilon(0)$ matches that of the solid dielectric pins $\left(\epsilon_{\mathrm{r}}=2.05\right)$. However, such an approach fixes the dielectric loss per unit length and requires that the overall dielectric loss must be adjusted solely by the choice of the total length. Alternately, the inductive reflections can be minimized by using thin dielectric supports of small cross-sectional area.

The $1.55 \mathrm{~m}$ and $3.10 \mathrm{~m}$ transmission lines have identical crosssectional dimensions and dielectric supports. The inner conductor diameter is $0.476 \mathrm{~cm}(3 / 16$ inch) which yields a characteristic impedance of $50 \mathrm{ohms}$ when the relative dielectric constant is 2.95. Using a 1.14 molal solution of 2 -heptanone in heptane, results in a relative dielectric constant of 2.54 and a characteristic impedance of $53.8 \mathrm{ohms}$. The dielectric supports are thin $(1.59 \mathrm{~mm}, 1 / 16$ inch) triangular shaped TFE wafers, Fig. 2.3. The uniformity of the resultant transmission line is illustrated by the TDR signature of the line filled with a 1. 14 molal solution of 2-heptanone in normal heptane, Fig. 2. 4. Inspection of the TDR signature shows that the relative reflected voltage is 0.0385 which corresponds to a characteristic impedance of $53.9 \mathrm{ohms}$. The inductive reflections due to the dielectric supports are negligible.

The transmission line physical dimensions and equivalent circuit parameters are summarized in Table 2. 1. The capacitance values are given for air $\left(\epsilon_{\mathrm{r}}=1\right)$ and for a 1.0-molal solution of 2-heptanone in normal heptane. The skin-effect metal loss coefficients are given for the brass and copper conductors ( 1.55 and 3.10 meter lines) and for the 
copper conductors ( 4.65 meter line).

\subsection{Coaxial Connector/Adapter Design and Parameters}

For a vertically positioned transmission line physically te rminated in an unmodified commercial adapter (Female Type $\mathrm{N}$ to $7 / 8$-inch rigid 50 ohm line), the lower adapter will trap a six millimeter column of liquid while the upper one approximately the same amount of air. The lower trapped polar-liquids would contaminate subsequent solutions; the upper void would present an undesirable transmission line section with a characteristic impedance higher than that of the liquid-filled section. Therefore, special adapters from Type $N$ (female) to $7 / 8$-inch rigid line have been designed and constructed for the $1.55 \mathrm{~m}, 3.10 \mathrm{~m}$, and $4.65 \mathrm{~m}$ trans mission lines to allow complete filling and draining of the liquids.

The adapter for the $4.65 \mathrm{~m}$ line consisted of a commercial adapter that had been mechanically modified to provide complete drainage and venting of the transmission line. No modifications were made to optimize the pulse response of the connector when filled with liquid. A commercial adapter with a modified drainage path is shown on the left in Fig. 2.5 and again with the internal structure of this adapter visible, in Fig. 2.6A (note the two O-ring seals for inner and outer conductors). A brass cube, silver-soldered adjacent to the Type $\mathrm{N}$ female end, has been drilled for drainage and tapped to accept 1/8inch male pipe thread. The original vent for pressurizing the line now sealed is shown in both photographs.

The adapters for the $1.55 \mathrm{~m}$ and $3.10 \mathrm{~m}$ transmission lines, in addition to adequate drainage properties, are designed to confine the liquids to the uniform line section only, thus improving the pulse response of the transition at either end between the 50-ohm external line sections and the liquid-filled section. One of these adapters is shown on the right in Fig. 2.5 with the drainage vent plugged for shipment. 
The adapter has been constructed from a modified Type $N$ (female) threaded panel connector $(U G-680 / U)$, and a brass flange meeting EIA standards except for thickness. The surface of this adapter facing the polar-liquids in operation can be seen in Fig. 2.6B. The drain plug has been removed in this view. The two original flats on the Type $N$ connector designed for a 9/16-inch wrench now serve as the beginning of the drain and fill apertures. An internal connection to the drain is not visible. Fig. 2. 7 is an engineering drawing of the adapter.

The TDR signatures for the new connectors attached to their respective liquid-filled transmission lines are shown in Figs. 2.8 and 2.9. The $4.5 \mathrm{~m}$ line contains a 1.0 -molal solution of 2-heptanone in heptane while the $1.55 \mathrm{~m}$ or $3.10 \mathrm{~m}$ line contains a 1.14 molal solution. The TDR* signatures can be characterized in terms of lumped inductive and capacitive elements, Figs. A-3 and A-7, by applying the following equations:

$$
\begin{aligned}
& L=\frac{\left(R_{01}+R_{02}\right)^{2}}{2 R_{01}} \frac{e_{m}}{m} \\
& C=\frac{R_{01}}{2\left[\frac{R_{01} R_{02}}{R_{01}+R_{02}}\right]^{2}} \frac{e_{m}}{m}
\end{aligned}
$$

Equations (2.12) and (2.13) provide a means for estimating the inductance or capacitance of a discontinuity at the junction of two transmission lines of different characteristic impedances, $R_{01}$ and $R_{02}$ being the characteristic impedances for the input and output transmission lines, respectively. $e_{m}$ is the peak value of the reflected voltage measured from the steady state level of the reflected voltage $e_{r}(\infty)$.

\footnotetext{
* See Appendix A. 1 for a complete description of time domain reflectometry (TDR) and definitions of the various parameters used in this TDR discussion.
} 
When $e_{m}$ is greater than $e_{r}(\infty)$, the discontinuity is inductive and (2.12) is used; for $\mathrm{e}_{\mathrm{m}}<\mathrm{e}_{\mathrm{r}}$, capacitance is present and (2.13) is used. $m$ is the slope of the incident voltage finite-ramp, $e_{i}(t)$. These eqs. result from the application of the bandwidth limiting conditions,

$$
\left(R_{01}+R_{02}\right)>>|s L|
$$

and

$$
\left(G_{01}+G_{02}\right)>>|s C|
$$

respectively, to the general solution for the transmission line voltage in the presence of an L or C discontinuity. The incident voltage is a finite ramp of the form

$$
e_{i}(t) \quad \begin{cases}m t, & 0 \leq t \leq T \\ m T & T \leq t \infty\end{cases}
$$

In practice, $m$ is the maximum rate of voltage change in the first transition region of the incident transition function,

$$
\left.\frac{\mathrm{de}_{i}(t)}{\mathrm{dt}}\right|_{\max }=\mathrm{m}, \text { volts } / \text { sec. }
$$

When $R_{01}=R_{02},(2.12)$ and (2.13) reduce to the usual TDR equations for a transmission line of characteristic impedance $R_{0}$ with a given $L$ or C discontinuity $[2,3]$,

$$
L=\frac{2 \mathrm{R}_{0} \mathrm{e}_{\mathrm{m}}}{\mathrm{m}}
$$

and

$$
C=\frac{2 e_{m}}{R_{0} m}
$$


When there are two successive discontinuities, the TDR signature in the S domain will be of the form

$$
E(s)=\frac{E_{g}(s)}{2}\left[1+\rho_{1} e^{-2 s T_{1}}+\rho_{2}\left(1-\rho_{1}^{2}\right) e^{-2 s\left(T_{1}+T_{2}\right)}+\ldots\right]
$$

where $\rho_{1}, \rho_{2}, T_{1}$, and $T_{2}$ denote the first-discontinuity reflection coefficient, the second-discontinuity reflection coefficient, the propagation time between the TDR reference plane and the first discontinuity, and the propagation time between the first and second discontinuities, respectively. Consequently, it is seen that the contribution from the second discontinuity is weighted by the factor

$$
\mathrm{W}=\left(1-\rho_{1}^{2}\right) \text {. }
$$

Inspection of the TDR signature for the $4.65 \mathrm{~m}$ line connector, Fig. 2. 8, shows that three discontinuities are present; $\mathrm{e}_{\mathrm{m} 0}, \mathrm{e}_{\mathrm{ml}}$, and $\mathrm{e}_{\mathrm{m} 2}$ correspond successively to a capacitive and two inductive discontinuities, respectively. The capacitive discontinuity is located at the junction of a $50 \mathrm{ohm}$ and a $32.5 \mathrm{ohm}$ transmission line; the refore (2.13) is used to compute $C$ from the peak reflected voltage $e_{\mathrm{m} 0}$.

The subsequent discontinuities are seen through the first one with the weighting factor of $1-\rho_{1}^{2}$. The first reflection coefficient is bandlimited such that $\left(G_{01}+G_{02}\right)>>|s c|$; hence $\rho_{1}$ is approximately,

$$
\rho_{1} \approx \frac{33-50}{33+50} \simeq-0.2
$$


which gives a weighting function of

$$
1-\rho_{1}^{2}=0.96
$$

Accordingly, the peak voltage values $\mathrm{e}_{\mathrm{ml}}$ and $\mathrm{e}_{\mathrm{m} 2}$ produced in the $32.5 \mathrm{ohm}$ line should be multiplied by $1 / 0.96$ to remove the weighting due to the first discontinuity. Similarly, there is a weighting by the second discontinuity of the reflected voltage from the third discontinuity; however, since the duration of the reflected voltage from the second discontinuity is much shorter than that of the third, the weighting factor of the second discontinuity is negligible. Because the inductive dis continuities generating $\mathrm{e}_{\mathrm{m} 1}$ and $\mathrm{e}_{\mathrm{m} 2}$ are located in the uniform trans mission line of $R_{0}=32.5$ ohms, in principle, (2.18) should be used with $\mathrm{R}_{0}=32.5$ and the peak voltages modified to $\mathrm{e}_{\mathrm{ml}} / 0.96$ and $\mathrm{e}_{\mathrm{m} 2} / 0.96$. However, (2.12) will give the same result. This can be demonstrated by dividing $\mathrm{e}_{\mathrm{m}}$ in (2.18) and (2.19) by the bandlimited form of (2.21); the results will be (2.12) and (2.13), respectively. The reason why these results are the same is that $\mathrm{e}_{\mathrm{m}}$ in both (2.12) and (2.13) is referred to the steady state value of the reflected voltage. Applying (2.12) and (2.13) to the TDR signatures shown in Figs. 2.8 and 2.9, yields the discontinuity capacitances and inductances for the two connectors, Fig. 1.6; these values are given in Table 2.2.

To construct equivalent networks for the connectors requires the specification of the position of each discontinuity. This is done by considering the time delay between each reflection and putting a corresponding lossless transmission line length between each successive discontinuity element. However, when the discontinuities are very close to each other in the sense that the propagation time between them is very small compared to the over all system transition time, the transmission line elements may be deleted for a first approximation. This yields a 
lumped circuit approximation for the connector network. Fig. 2.10 shows the lumped circuit equivalent networks for the $1.55 \mathrm{~m}$ and $3.10 \mathrm{~m}$, and $4.65 \mathrm{~m}$ lines; the elements are denoted by the $\pi$-network parameters (Fig. 1.6) $Z_{2}, Z_{3}, Z_{4}$ in Table 2.2.

\subsection{Liquid Dielectric Preparation and Parameter Measurements}

The mathematical form and source impedance of the generated waveform is mainly determined by the bandwidth limiting properties of the losses produced in the liquid polar dielectric which fills the dielectric space of the uniform coaxial transmission line.

Three different ketones were used as solutes with heptane as the solvent. The ketones were 2-butanone, 2-heptanone, and 2-octanone. Preparation and maintenance of these solutions require some care as all four organic liquids a re quite volatile and are excellent solvents. To minimize evaporation errors, the molal solutions of $0.25,0.50$, 1. 0 , and $2.0 \mathrm{~m} \mathrm{2-butanone,} \mathrm{2-heptanone,} \mathrm{and} \mathrm{2-octanone} \mathrm{were} \mathrm{pre-}$ pared directly into their containers on a laboratory single-pan balance, first weighing out the required fractional gram-molecular-weight of the ketone and adding a kilogram of heptane.

The first solutions prepared were stored in polyethylene containers which proved to be an unfortunate choice for the storage containers. Serious changes in the $1 \mathrm{kHz}$ dielectric constants of the solutions were measured in the weeks following preparation. The changes were apparently caused by a differential diffusion of solvents and solutes through the polyethylene containers in which the newly prepared solutions were stored. Glass containers were not used and were ruled out because of the safety hazard involved in breakage with flammable liquids being present. By changing to metal storage containers the evaporation induced changes in the dielectric constants of the stored liquids were eliminated. 
The static dielectric constant values were determined by $1 \mathrm{kHz}$ measurements. Since the Debye relaxation times of each resultant solution is about 5 picoseconds, then at $1 \mathrm{kHz}$ the relative dielectric constant will be essentially equal to the static value.

$\mathrm{U}$ sing a precision transformer ratio-arm bridge at $1 \mathrm{kHz}$, the open-circuit capacitance of the 15-foot transmission line was measured when filled sequentially with air, heptane, polar solutions, and benzene. The relative static dielectric constants, $\epsilon_{r}(0)$, of the polar solutions listed in Table 2. 3 were computed from these measurements using the dielectric constant of heptane as a standard reference, with benzene serving as a secondary reference. A schedule of rinsing the transmission line with heptane and drying with forced air and dry nitrogen between different solutions was employed. The rinse and dry schedule is necessary to prevent contamination of the heptane and benzene reference standards of capacitance as was noted by the continual increase in dielectric constant of the heptane rinse solution.

Verification of the handbook [4] value for the dielectric constant of heptane was performed in two stages. First, an interpolation of $\epsilon_{r}$ and its temperature coefficient $\alpha$ for each of five other alkanes (pentane, hexane, octane, nonane, and decane) gave a value for $\epsilon_{r}$ and $\alpha$ for heptane. This interpolation was necessary because of the unfortunate omission of heptane from the table of the dielectric constants and their temperature coefficients of organic liquids in this handbook [5]. The temperature coefficient of $\epsilon_{r}$ for heptane was of primary interest since the reference waveform generator is normally used at a temperature other than $20^{\circ} \mathrm{C}$ for which the tables are given. For a liquid dielectric consisting of non-polar molecules, the dielectric constant decreases with an increase in temperature because of the decrease in the number of molecules per unit volume. Figure 2.11 shows 
the interpolated values of $\epsilon_{\mathrm{r}}$ and $\alpha$ for heptane; at $23^{\circ} \mathrm{C}, \epsilon_{\mathrm{r}}=1.919$ and $\alpha=-0.148 \times 10^{-2}$. A negative temperature coefficient for the polar solutions also was noted experimentally which is consistent with the $1 / \mathrm{T}$ temperature dependence of the molecular polarization in the Debye model for weak polar solutions [6].

Second, the above values were verified by using benzene as a dielectric standard because $\epsilon_{\mathrm{r}}$ and $\alpha$ are well established $\left(\epsilon_{\mathrm{r}}=2.278\right.$ (@ $23^{\circ} \mathrm{C}$ and $\left.\alpha=0.2 \times 10^{-2}[4]\right)$. The verification was accomplished by filling the line with benzene and measuring the resultant capacitance. This capacitance value was compared with that obtained with heptane and verified the values of $\epsilon_{r}$ and $\alpha$ for heptane within 0.3 percent.

Direct measurements of $\epsilon_{r}$ for heptane, benzene and the polar solutions were attempted on a precision $1 \mathrm{kHz}$ bridge using a short section of air-spaced coaxial transmission line as a test cell. The desired precision in these measurements was not achieved because of evaporation problems with the small amounts of liquids involved and the difficulty encountered in keeping the test cell connectors uncontaminated by the polar solutions.

In Section 2. 1 it was pointed out that the admittance/m $\mathrm{Y}(\mathrm{s})$ can also be expressed in the form $A \in(s)$. Consequently, (2.2) through (2.4) can be written as

$$
\begin{aligned}
Y(s) & =A \epsilon_{0} \epsilon_{r}(s) \\
C_{1} & =A \epsilon_{0} \epsilon_{r}(\infty) \\
C_{1}+C_{2} & =A \epsilon_{0} \epsilon_{r}(0)
\end{aligned}
$$

and

$$
Y(s)=A \epsilon_{0}\left[\epsilon_{r}(\infty)+\frac{\epsilon_{r}(0)-\epsilon_{r}(\infty)}{1+s \tau}\right]
$$

where $\epsilon_{0}$ is the dielectric permittivity of free space, farads/meter. 
Comparing (2.22) with (2.25) yields the frequency dependent relative dielectric function,

$$
\epsilon_{r}(s)=\epsilon_{r}(\infty)+\frac{\epsilon_{r}(0)-\epsilon_{r}(\infty)}{1+s \tau}
$$

For a given solution and solvent the $1 \mathrm{kHz}$ capacitance measurements provide the data for calculating $\epsilon_{r}(0)$ and $\epsilon_{r}(\infty)$; the former is the static relative dielectric constant of the binary solution while the latter is that of the solvent.

The reason why the high frequency limit for the solution's relative dielectric function (2. 26) is given by the solvent's static relative dielectric constant is that the induced molecular dipole orientation of the polar solute molecules can not follow a high frequency electric field for frequencies much greater than $1 / \tau$. The major contribution from the solute molecules to the dielectric polarization at high frequencies is their electronic polarization. Accordingly, the relative dielectric constant of the solute at very high frequency is about the same as that for the solvent as the solvent polarization is totally electronic. Consequently, the high frequency limit for the dilute solution dielectric polarization is electronic and is essentially equal to that of the solvent. Because the solvent molecules are non-polar, the solvent polarization is solely electronic and frequency independent. Therefore, measurements at $1 \mathrm{kHz}$ establish the value of the solvent's relative dielectric constant which in turn is equal to the high frequency limit of $(2.26), \epsilon_{\mathrm{r}}(\infty)$.

The one remaining parameter to be determined is the Debye relaxation time $\tau$. It is established by a computer program iteration using (1.6) which matches the predicted response with the observed experimental response. Here, the overall system predicted response is compared to the observed experimental response, Figs. (2.13) and (2. 14): 
The value of $\tau$ is selected which causes the predicted response to fit the experimental data when the Debye relaxation model is valid, the value of $\tau$ which establishes the fit to the experimental data is in fact the Debye relaxation time, Fig. 2.14.

\subsection{Available-Waveform Computation/Evaluation}

The computation of the Available-Waveform and its inherent squared-error is based upon the following assumptions:

1) The planar skin effect model accurately represents the conductor surface impedance.

2) The Debye model accurately represents the dielectric function $\epsilon$ (s).

3) The high frequency limiting value of the relative dielectric function $\epsilon_{\mathrm{r}}(\infty)$ equals 1.92 , the static value for heptane.

4) The connector equivalent circuit is valid for the system transition-times under consideration.

These assumptions if not valid can always be removed by using more accurate models based upon experimental data. Consequently, they offer no fundamental limitations to the methods used here. The procedure for computing the Available-Waveform and its inherent squarederror consists of the following steps:

1) Acquire and digitize the experimentally observed driving function $e_{d I}(t),(1.1)$ Fig. 2. 12 .

2) For the given transmission line and connectors, acquire and digitize the experimentally observed output voltage $e_{d 2}(t),(1.6)$ Fig. 2.13, dashed curve. 
3) Using the numerical values for $e_{d l}(t)$, the applicable set of transmission line and connector parameters (Tables 2.1 and 2.2), and estimated values of $\tau$ in equation (1.6), iteratively determine $\tau$ by repeatedly computing $\overline{\mathrm{e}_{\mathrm{d} 2}(\mathrm{t})}$ until the computed value of $\overline{e_{d 2}(t)}$ over-lays the observed value $e_{d 2}(t)$. The solid curve in Fig. 2.13 is $\overline{\mathrm{e} 2}(\mathrm{t})$ computed for $\tau=5.52$ picoseconds. Fig. 2. 14 shows the resultant overlay of $\bar{e}_{\mathrm{d} 2}(t)$ and $e_{d 2}(t)$, and the squared difference between the two curves, $\Delta^{2}(t)$. When $\tau$ is finally determined, all the parameters of $\gamma(s)$ and $Z_{0}(s)$ are determined.

4) Compute the Available- $V$ aveform $e_{a}(t),(1.11)$ and compare it to $e_{0}(t)$ in accordance with the instructions following (1.12). $e_{a}(x)$ is the solid curve while $e_{0}(x)$ is the dashed curve in Fig. 2. 15.

5) Compute the squared-difference $\Delta^{2}(x)$ between $e_{a}(x)$ and $\mathrm{e}_{0}(\mathrm{x}),(1.12)$

In summary, the above procedure is illustrated for the 4.65 meter line in Figs. 2.12 through 2.16. The data illustrated in Figs. 2. 15 and 2. 16 are the calibration data for the 4.65 meter Reference-Waveform Generator and are tabulated in Appendix A.2. Table 2. 4 summarizes the electrical characteristics of the $4.65 \mathrm{~m}$ Reference Waveform Generator . 


\begin{tabular}{|c|c|c|c|}
\hline \multirow{2}{*}{ Parameter } & \multicolumn{3}{|c|}{ Transmission Line } \\
\hline & $1.55 \mathrm{~m}$ & $3.10 \mathrm{~m}$ & $4.65 \mathrm{~m}$ \\
\hline$l$, Length of uniform section, $\mathrm{m}$ & 1.526 & 3.050 & 4.660 \\
\hline $\mathrm{r}_{2}$, Outer conductor radius, $\mathrm{cm}$ & 0.997 & 0.997 & 0.997 \\
\hline $\mathrm{r}_{1}$, Inner conductor radius, $\mathrm{cm}$ & 0.238 & 0.238 & 0.433 \\
\hline L, External inductance, $\mathrm{nH} / \mathrm{m}$ & 286 & 286 & 166.8 \\
\hline $\mathrm{C}_{\mathrm{o}}$, Capacitance, air-filled, $\mathrm{pF} / \mathrm{m}$ & 38.43 & 38.31 & 66.32 \\
\hline $\begin{array}{l}C_{1} \text {, Capacitance, high frequency } \\
\text { limit (heptane), } \mathrm{pF} / \mathrm{m}\end{array}$ & 73.73 & 73.51 & 127.26 \\
\hline $\begin{array}{l}\mathrm{C}_{1}+\mathrm{C}_{2}, \text { Low frequency limit ( } 1 \\
\text { molal solution), } \mathrm{pF} / \mathrm{m} \\
\text { of } 2 \text { - Heptanone in Heptane }\end{array}$ & 94.73 & 94.44 & 163.50 \\
\hline $\begin{array}{l}\mathrm{K}, \text { Skin effect coefficient, } \mu \Omega / \mathrm{m} \\
(\text { radians } / \mathrm{sec})^{1 / 2}\end{array}$ & 17.5 & 17.5 & 7.86 \\
\hline Capacitance, benzene-filled, $\mathrm{pF} / \mathrm{m}$ & 87.50 & 87.22 & 151.01 \\
\hline
\end{tabular}


TABLE 2.2

CONNECTOR PARAMETERS

\begin{tabular}{|c|c|c|c|}
\hline \multirow{2}{*}{$\begin{array}{c}\text { Network } \\
\text { Impedances }\end{array}$} & Line Length and & \multicolumn{2}{c|}{ Solution } \\
Concentration \\
\cline { 2 - 4 } & $\begin{array}{c}1.55 \mathrm{~m} \\
(1.14 \text { Molal })\end{array}$ & $\begin{array}{c}3.10 \mathrm{~m} \\
(1.14 \mathrm{Molal})\end{array}$ & $\begin{array}{c}4.65 \mathrm{~m} \\
(1.0 \mathrm{Mola})\end{array}$ \\
\hline $\mathrm{Z}_{2}$ & $\infty$ & $\infty$ & $0.06 \mathrm{pF}$ \\
$\mathrm{Z}_{3}$ & $0.48 \mathrm{nH}$ & $0.48 \mathrm{nH}$ & $0.57 \mathrm{nH}$ \\
$\mathrm{Z}_{4}$ & $0.41 \mathrm{pF}$ & $0.41 \mathrm{pF}$ & $\infty$ \\
\hline
\end{tabular}


RELATIVE $1 \mathrm{kHZ}$ DIELECTRIC CONSTANTS OF POLAR SOLUTIONS AND NONPOLAR SOLVENTS AT $23^{\circ} \mathrm{C}$

Solution

2-butanone/heptane

2-heptanone/heptane

2-octanone/heptane

Heptane

Benzene
Concentration

$\epsilon_{r}(0)$
$0.25 \mathrm{~m}$
2. 04
0.50
2. 15
1.00
2. 39
2. 00
2. 92

0.25

2.06

0.50

2. 19

1. 00

2. 46

2. 00

2. 98

0.25

2. 06

0.50

2. 19

1. 00

2. 46

2. 00

2. 96

1.92

2. 28 
SUMMARY OF ELECTRICAL CHARACTERISTICS FOR 4.65 M REFERENCE WAVEFORM GENERATOR

Polar dielectric

Low frequency characteristic impedance

Time window of evaluation

10\%-90\% Transition times

Defined available waveform, t

Generator output voltage, $t_{0}$

Transition time difference $\%$,

$\frac{t_{0}-t_{a}}{t_{a}} \times 100$

Waveform uncertainty in a $4 \mathrm{~ns}$ time window, peak value of the squared difference
1 . 0 molal concentration of 2 -Heptanone in Heptane $\epsilon_{r}(0)=2.46$, $\epsilon_{\mathrm{r}}(\infty)=1.92, \quad \tau=3.52 \mathrm{ps}$.

32.5 ohms

$4 \mathrm{~ns}$

381 psec.

380.2 psec.

$-0.29 \%$

$5.76 \times 10^{-4}$ 


\section{OPERATING SYSTEM}

An operational Reference Waveform Generating System consists of two subsystems: (1) the electrical network for the waveform generation and (2) a waveform data acquisition system for calibration and evaluation of the generated waveform. The former subsystem is the subject of this report while the latter one is reported elsewhere [7]. In this chapter the overall physical structure of the electrical network will be presented along with a brief description of the waveform data acquisition subsystem.

\section{3. $1 \quad$ Electrical Network Physical Structure}

For operating convenience, the transmission-line sections of the waveform generators have been mounted vertically on a 24-foot (antenna) tower, their lower ends accessible for pressure-filling with the polar-liquid on one floor, their upper ends adjacent to the data acquisition equipment on the second level. The arrangement for filling the transmission lines with the polar solutions is diagramed in Fig. 3. 1. A photograph of the tower from near the lower-floor level, Fig. 3.2, shows the 15-foot line just visible on the right. Fig. 3. 3 shows a tunnel-diode pulse generator attached to the lower end of the transmission line; Fig. 3.4 shows a sampler for data acquisition attached to the upper end. Of course, the pulse generator and sampler may be interchanged, the transmission-line section is symmetrical.

A polar-liquid dielectric is placed within an adjacent steel paint tank, the tank is then pressurized with dry nitrogen gas at 5 to 10 psi forcing the polar liquid through the connecting tubing, special adapter, uniform line-section, into the overflow bottle. The pressurizing system (three tanks) can be seen in Fig. 3.3. The overflow bottle 
when approximately half-full, closes a microswitch indicating to the operator at the lower end by a panel light and buzzer that the transmission-line section is completely filled and the generator is ready for operation. The overflow bottle and microswitch are shown in Fig. 3. 4.

Exterior venting of the heptane- and ketone-laden fumes during the drying procedure described in Section 2.3 is necessary for safety. The 3/4-inch copper pipe visible just inside the left tower leg in Fig. 3.5 ventilates both the 15 -foot transmission line through its plastic overflow bottle and the paint tanks at the base of the tower through their common manifold. The venting manifold valves for the tanks can be seen in Fig. 3.2. The 5- and 10-foot generators can easily be filled visually without an electrical overflow-indicator; outside venting, however, is necessary.

\subsection{Waveform Data Acquisition System}

The data acquisition system is simply a sampling oscilloscope interfaced to a data storage system. A block diagram of the present operational system is shown in Fig. 3.6, [7]. This particular system can acquire and store 1000 sampled data values. The stored waveform is displayed on a CRT display unit; the data can be read out for processing on a time share computer or a general scientific computer. 


\section{SUMMARY}

The work reported here has for the most part answered the question as to how to utilize the step response of a band-limited network as a reference waveform.

Because perfect step generators and non-bandlimited connectors and oscilloscopes are not physically realizable, a priori knowledge of the shape of the reference waveform will be uncertain within definable limits over the waveform time window.

Accordingly, the defined reference waveform is given as the step response of a characterizable bandlimited network; its mathematical form and time domain response are known. The generated reference waveform (output waveform across the specified load) departs from the defined waveform. This departure is characterized by a squared-difference curve.

In this report, the reference-waveform generator was characterized in terms of the two quantities $e_{a}(t)$ and $\Delta^{2}(t)$ over the time window. $e_{a}(t)$ is the defined available waveform based upon the step response of a Debye Dielectric bandlimited transmission line operating into a $50 \mathrm{ohm}$ load and being driven by a perfect step generator of $50 \mathrm{ohm}$ impedance. $\Delta^{2}(t)$ is the departure between the generated waveform and the defined available waveform including the uncertainty due to oscillographic measurements. 


\section{REFERENCES}

[1] McCaa, Jr., W. D., and N. S. Nahman, Generation of reference waveforms by uniform lossy transmission lines, IEEE Trans. Instrum. Meas., IM-19, No. 4, 382-390 (Nov. 1970 ).

[2] Strickland, J. A., Time-Domain Reflectometry Measurements, 1st Ed. Tektronix, Inc., Beaverton, Ore., (1970).

[3] Time Domain Reflectometry, Application Note No. 62, HewlettPackard Co., Palo Alto, Calif., (1964).

[4] American Institute of Physics Handbook, 2nd Ed. McGraw-Hill Co., Inc., New York, N. Y., (1963).

[5] Handbook of Chemistry and Physics, $53 \mathrm{rd} \mathrm{Ed.,} \mathrm{The} \mathrm{Chemical}$ Rubber Co., Cleveland, Ohio, (1972).

[6] Debye, P., Polar Molecules, Dover Publications, New York, N. Y. , (1929)

[7] Gans, W. L., Block diagrams, schematic circuit diagrams, and operating instructions for the time domain measurement system. (To be published.) 

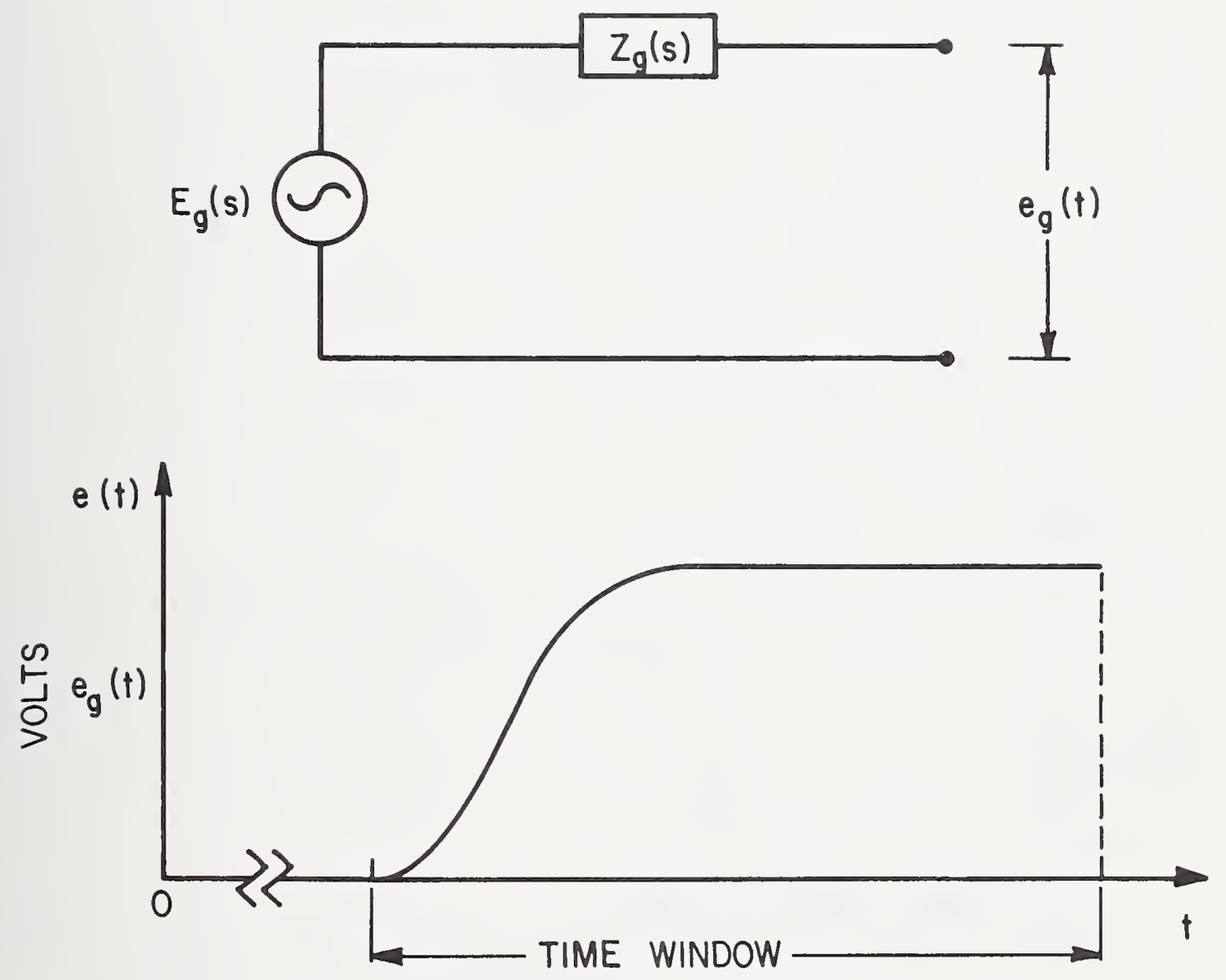

Figure 1.1 Reference waveform parameters. 

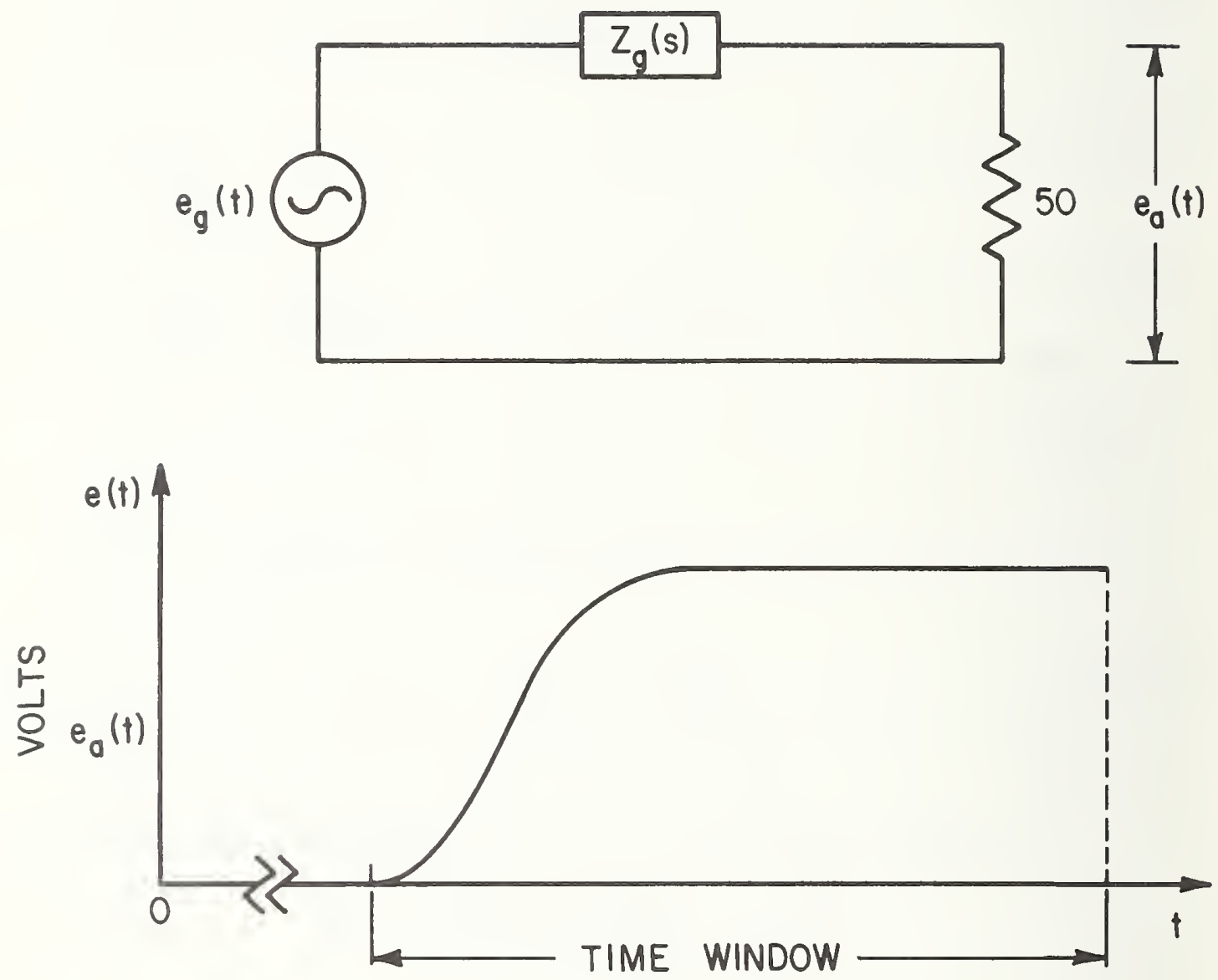

Figure 1.2 The available waveform e ${ }_{a}(t)$ across a $50 \mathrm{ohm} \mathrm{load.}$ 


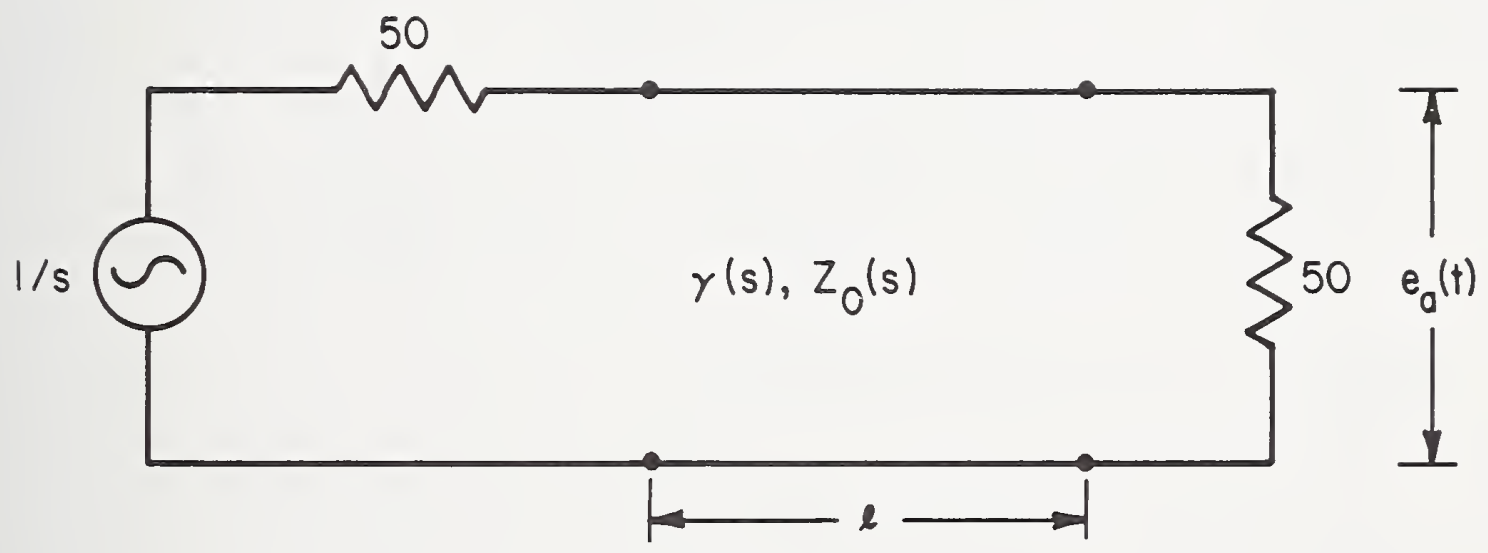

Figure 1.3 The defining network for the available waveform $e_{a}(t)$. 

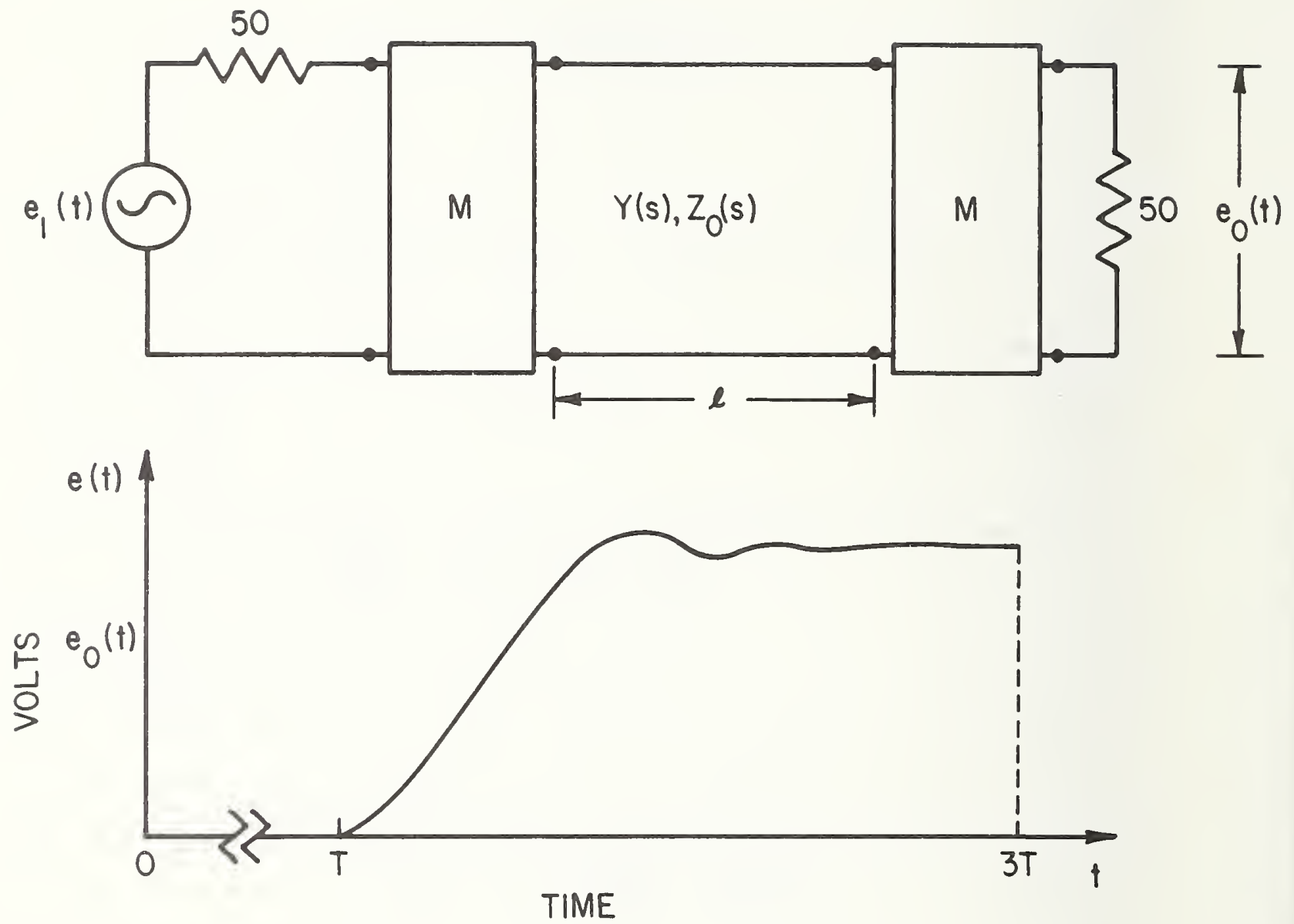

Figure 1.4 The generated waveform $e_{0}(t)$. 


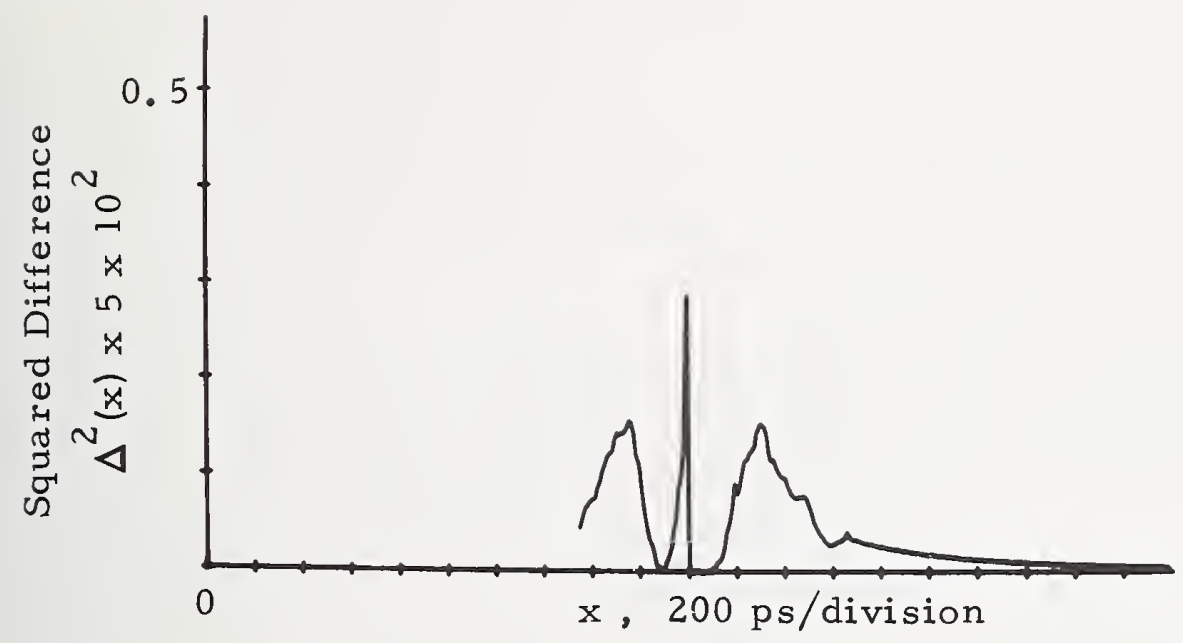

Figure 1.5A Squared difference of the output voltage $e_{0}(x)$ compared to the defined available waveform $e_{a}(x)$.

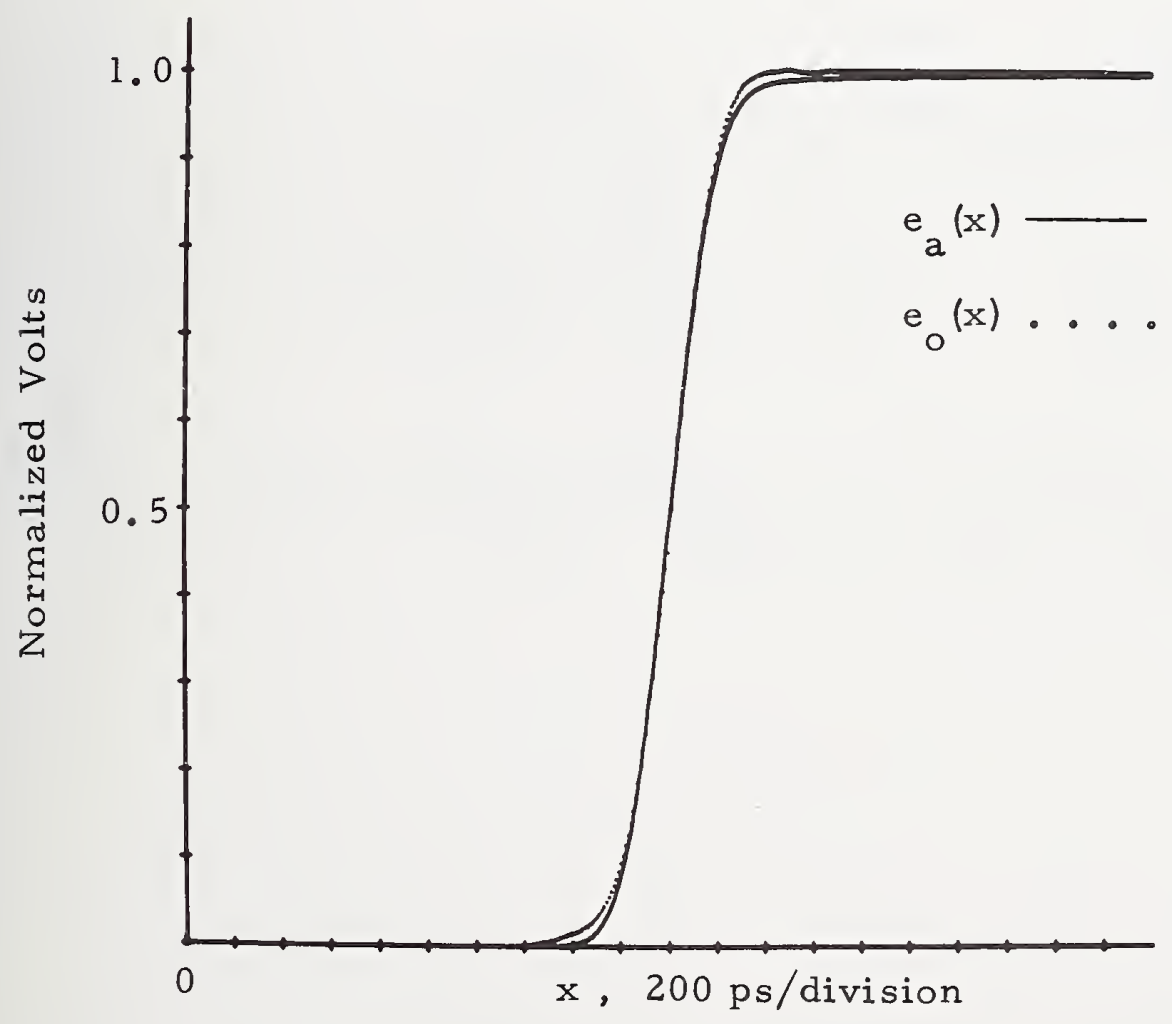

Figure 1.5B The output voltage $e_{0}(x)$ overlayed on the defined available waveform $e_{a}(x)$. 

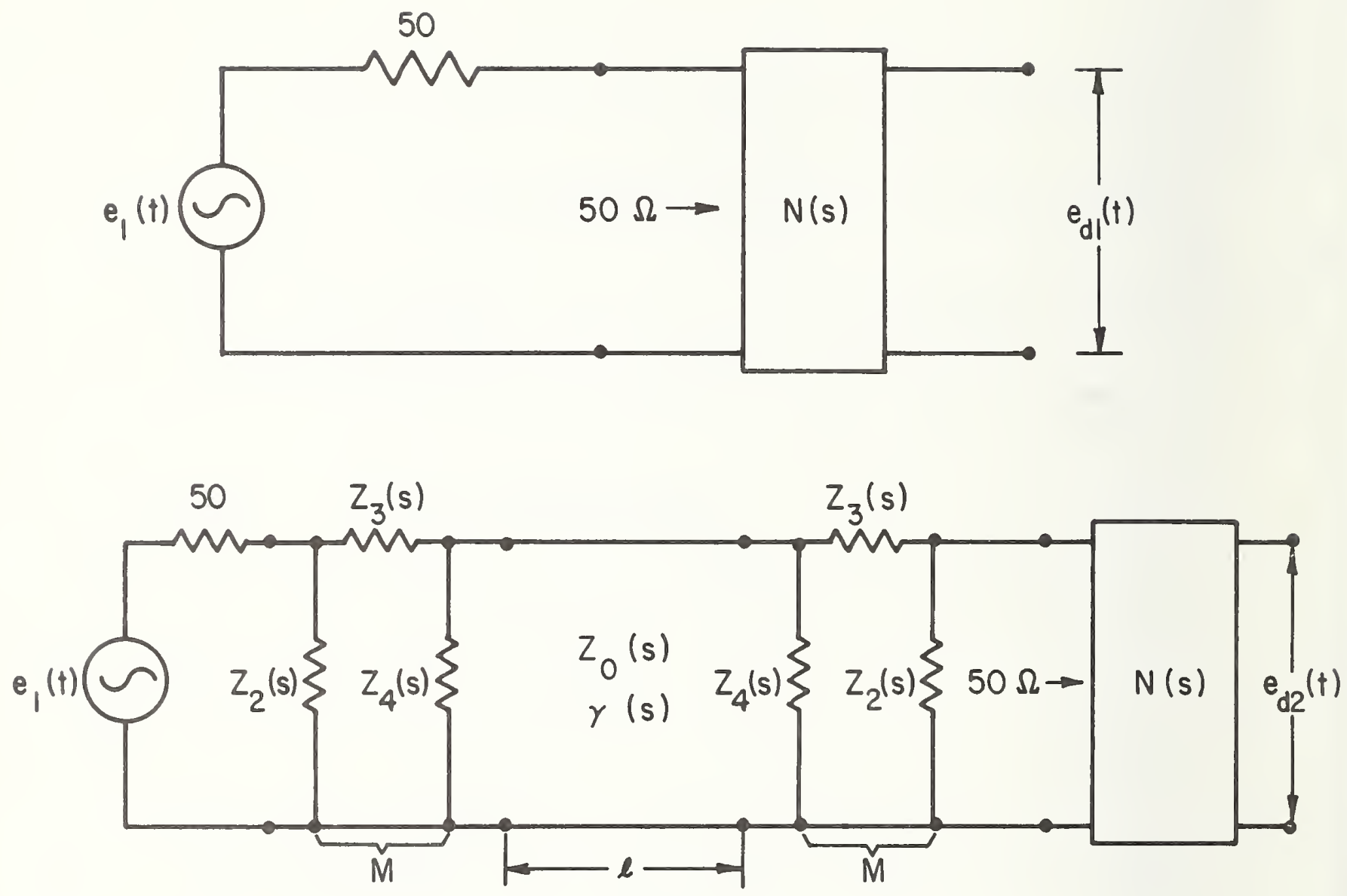

Figure 1.6 Time domain insertion method of calibration. 


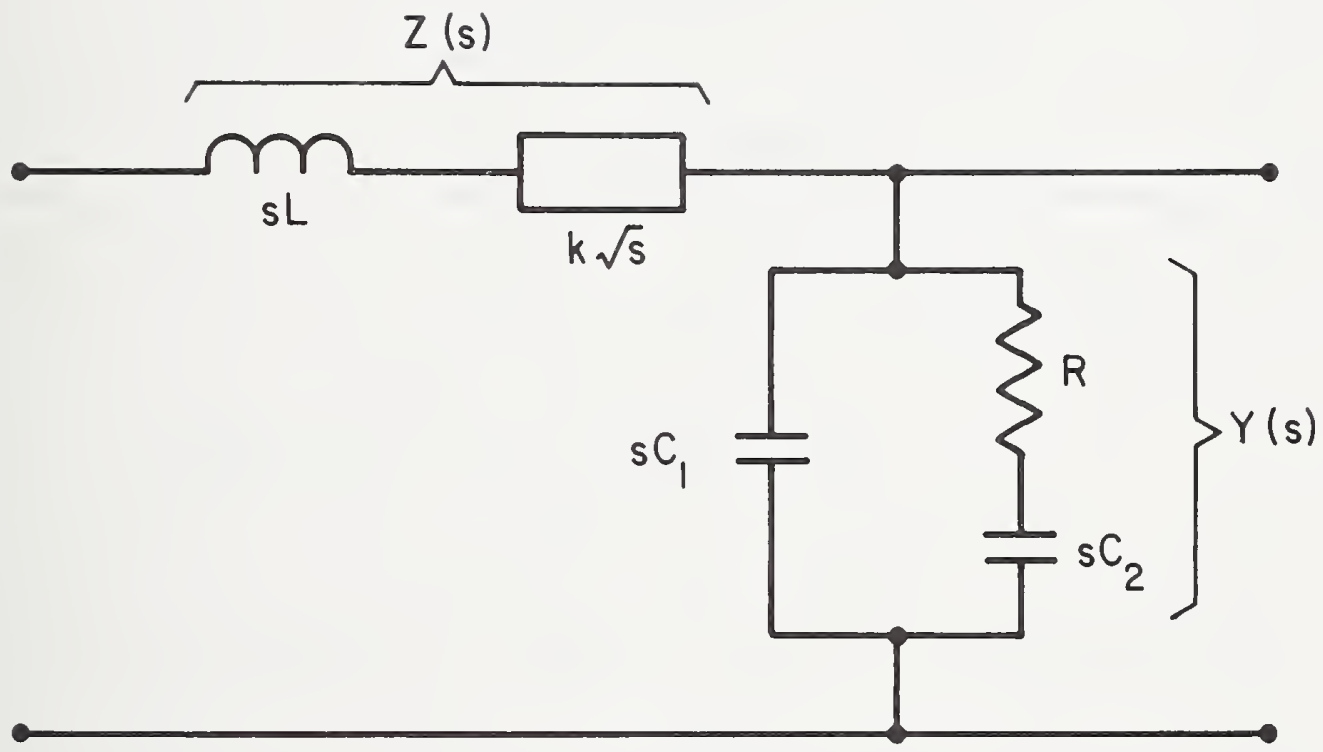

Figure 2.1 The equivalent circuit per meter for the uniform transmission line. 


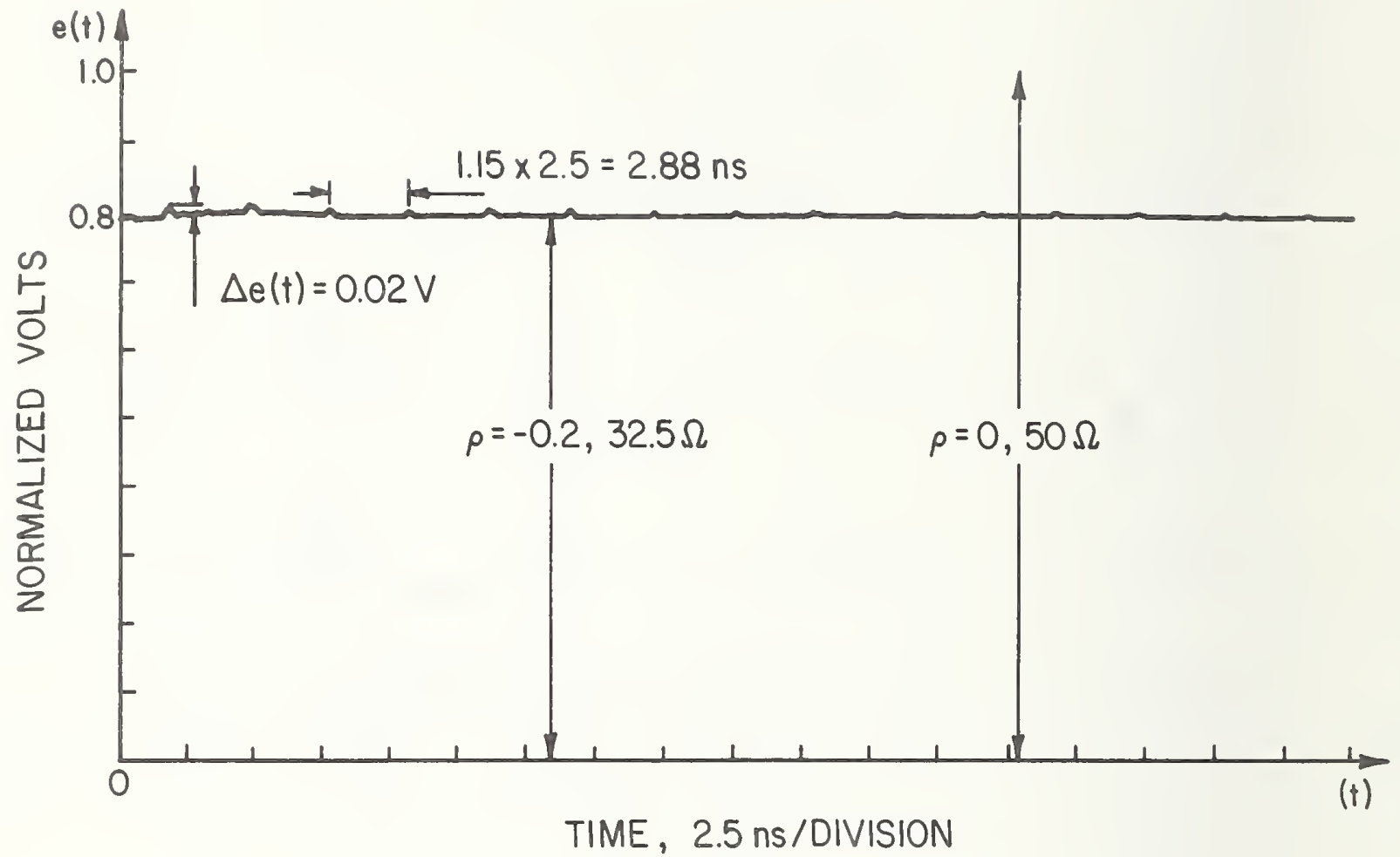

Figure 2.2 TDR signature of the nominally uniform $4.65 \mathrm{~m}$ transmission line filled with 1.0 molal solution of 2 -Heptanone in normal heptane. 


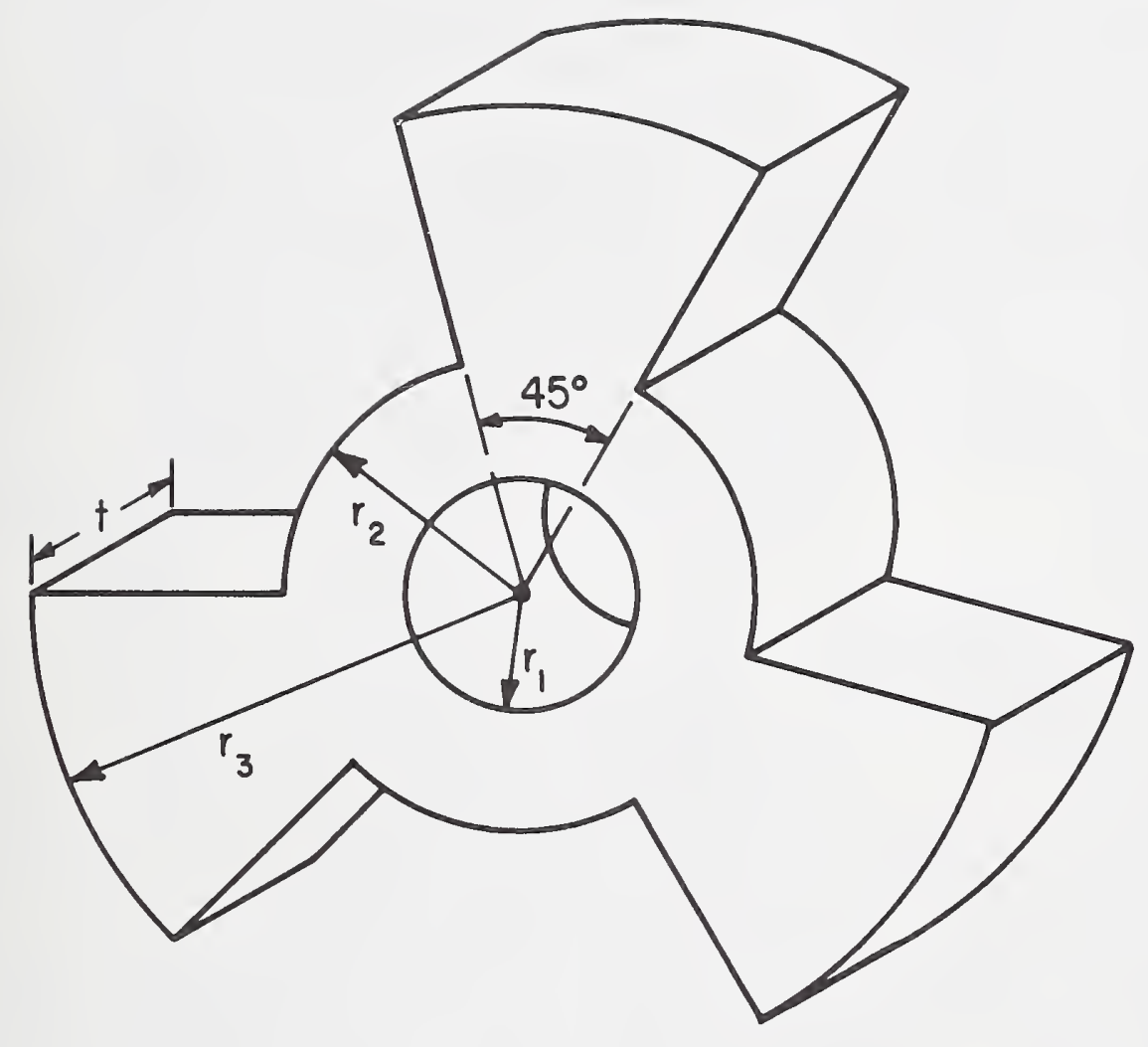

Figure 2.3 Dielectric support PTFE; $r_{1}=2.38 \mathrm{~m}, r_{2}=4.76 \mathrm{~mm}$, $r_{3}=9.97 \mathrm{~mm}, t=3.18 \mathrm{~mm}$. 


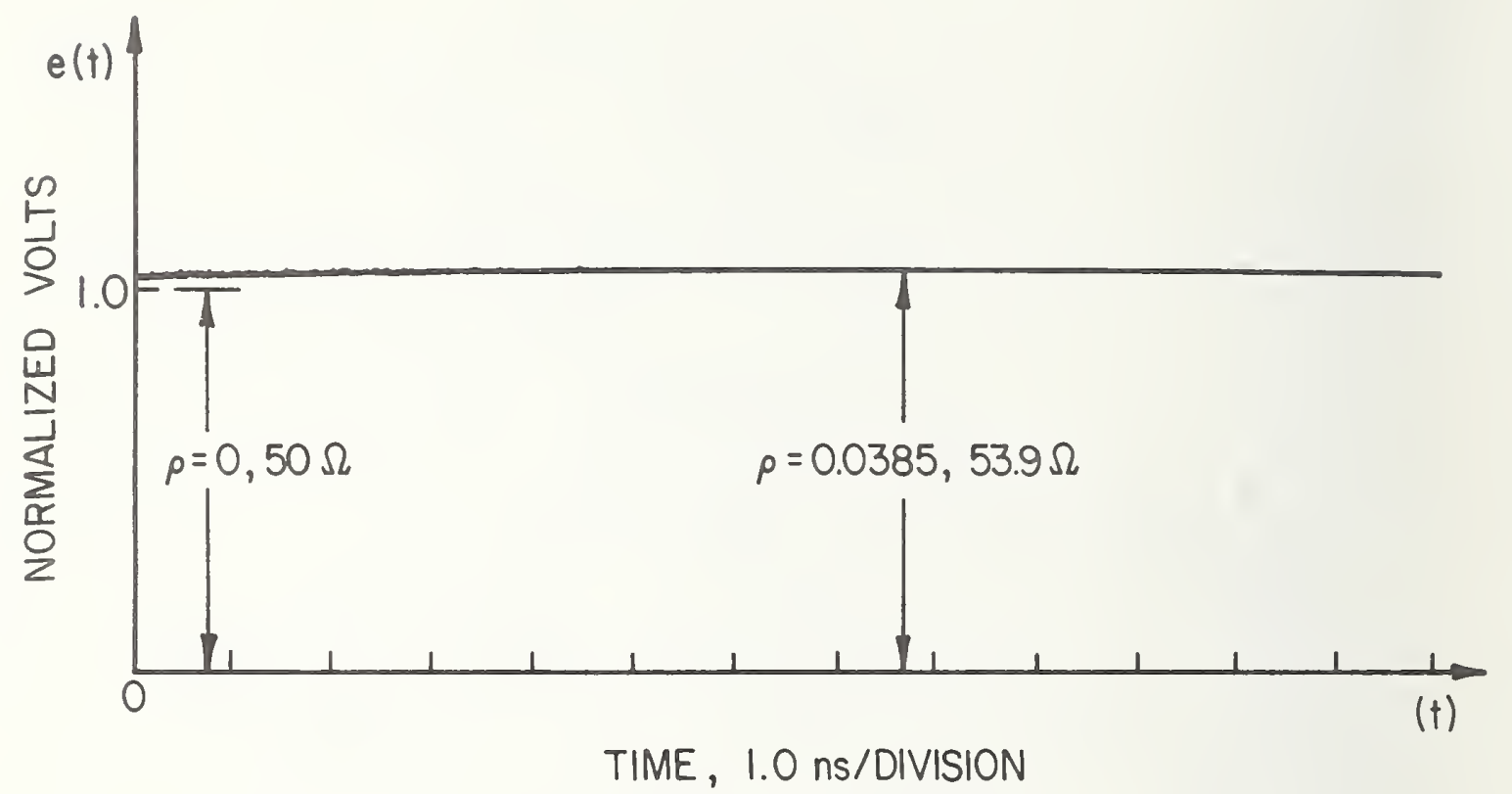

Figure 2.4 TDR signature of the nominally uniform $1.55 \mathrm{~m}$ transmission line filled with 1.14 molal solution of 2-Heptanone in normal Heptane. 


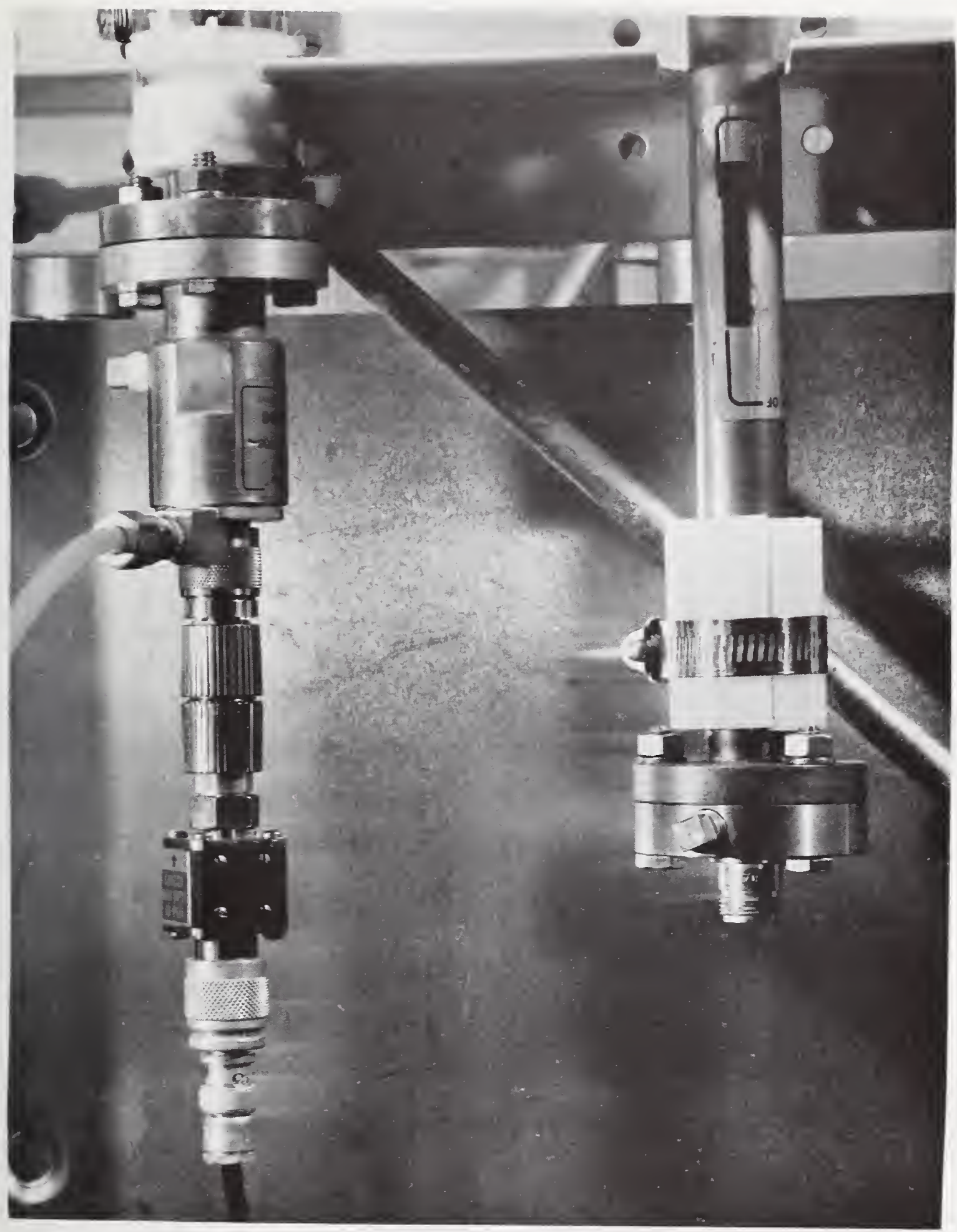

Figure 2.5 Modified and NBS adapters, external view. 


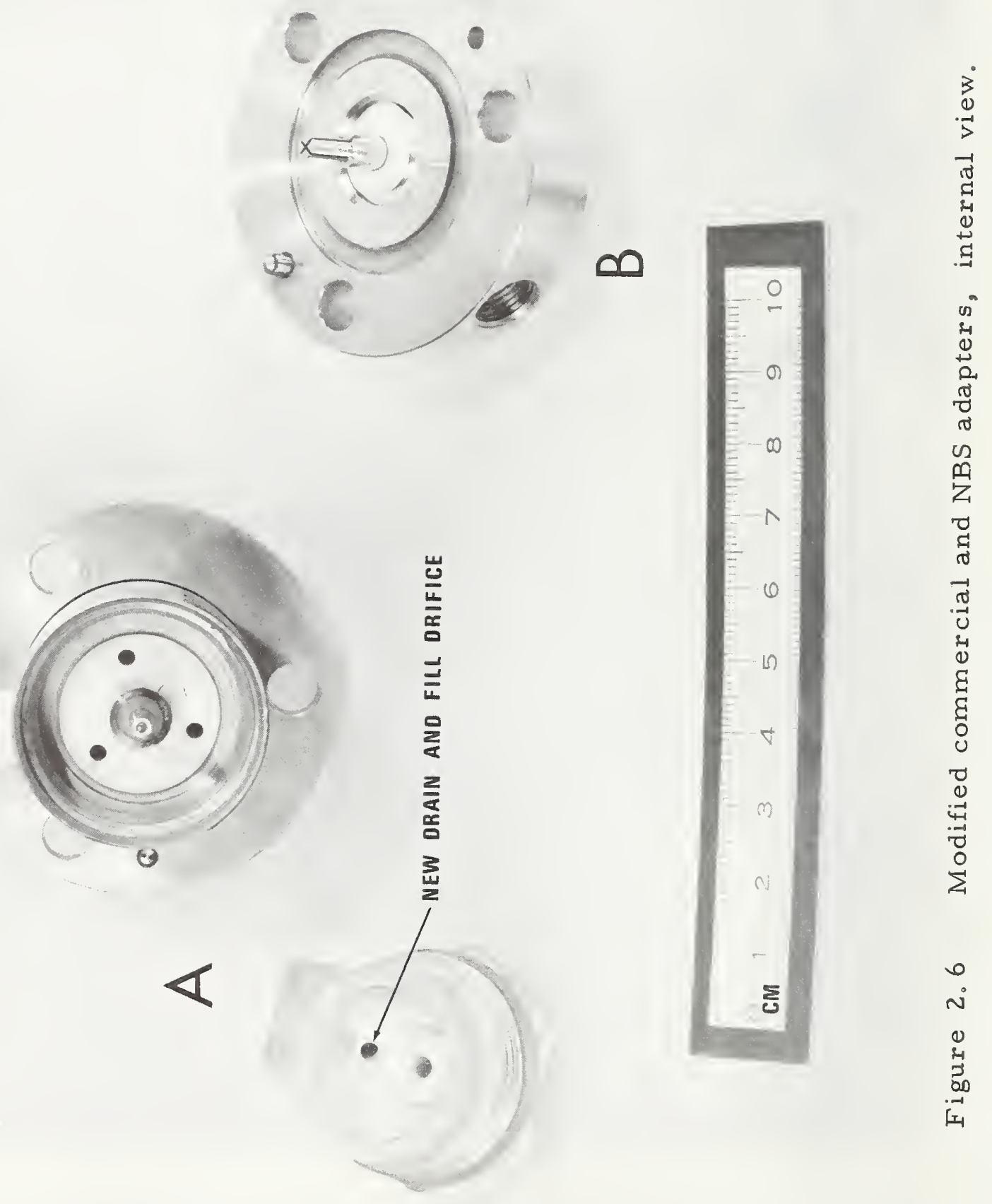



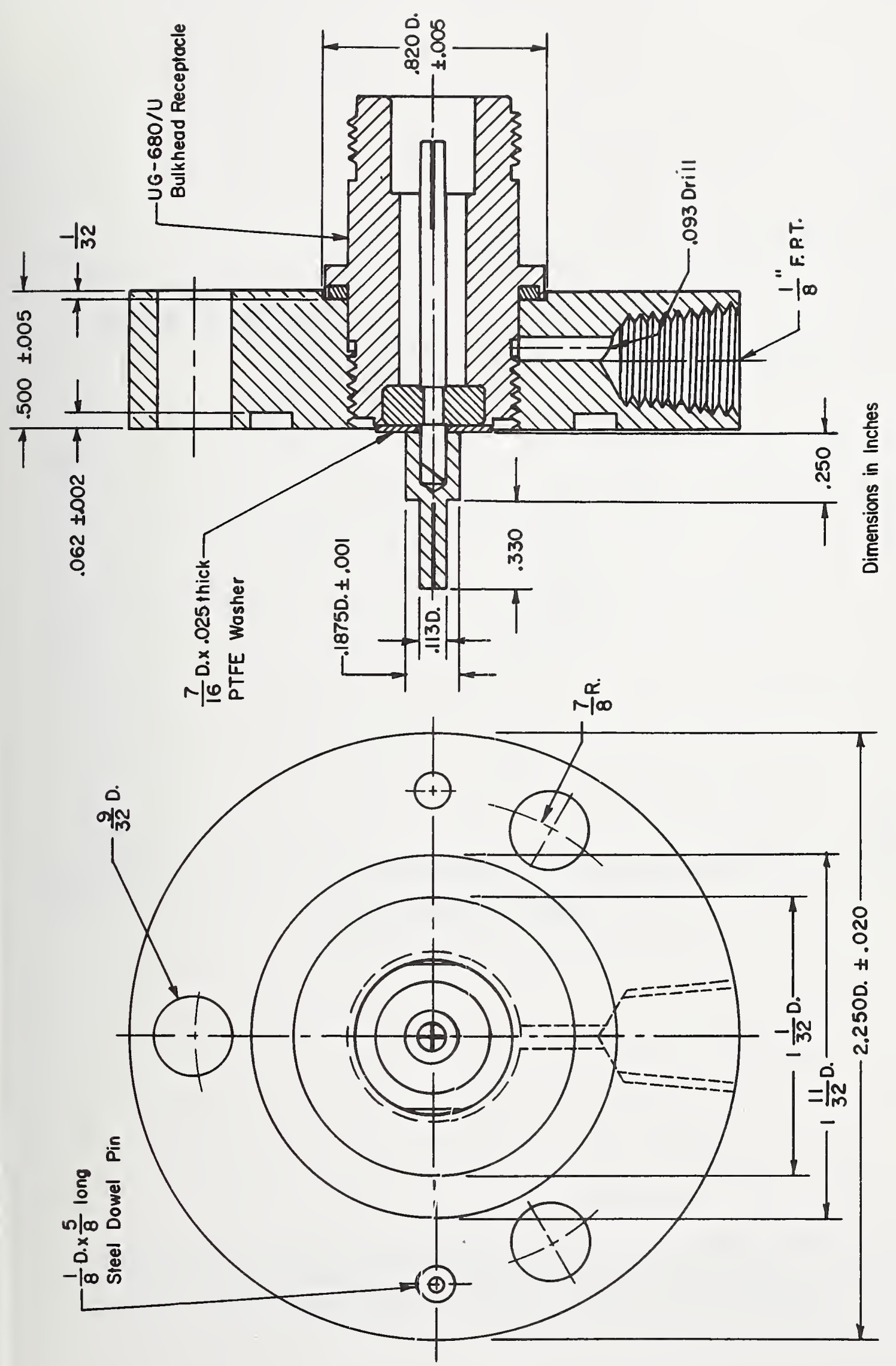

$\sim$
0
0
3
0
0
01
11 


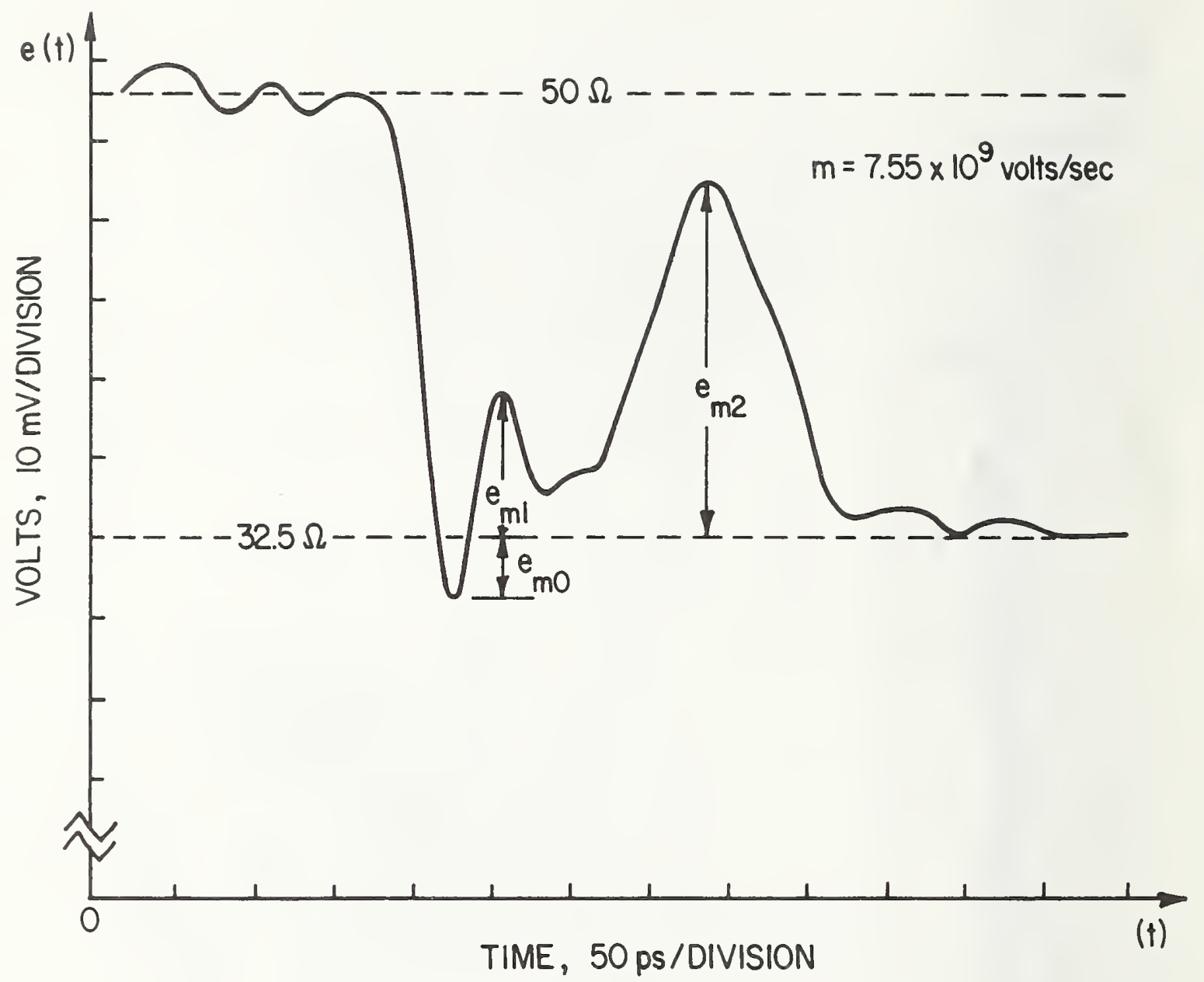

Figure 2.8 TDR signature of the connector used with the $4.65 \mathrm{~m}$ line filled with 1.0-molal solution of 2-Heptanone in $\mathrm{n}$-Heptane. 


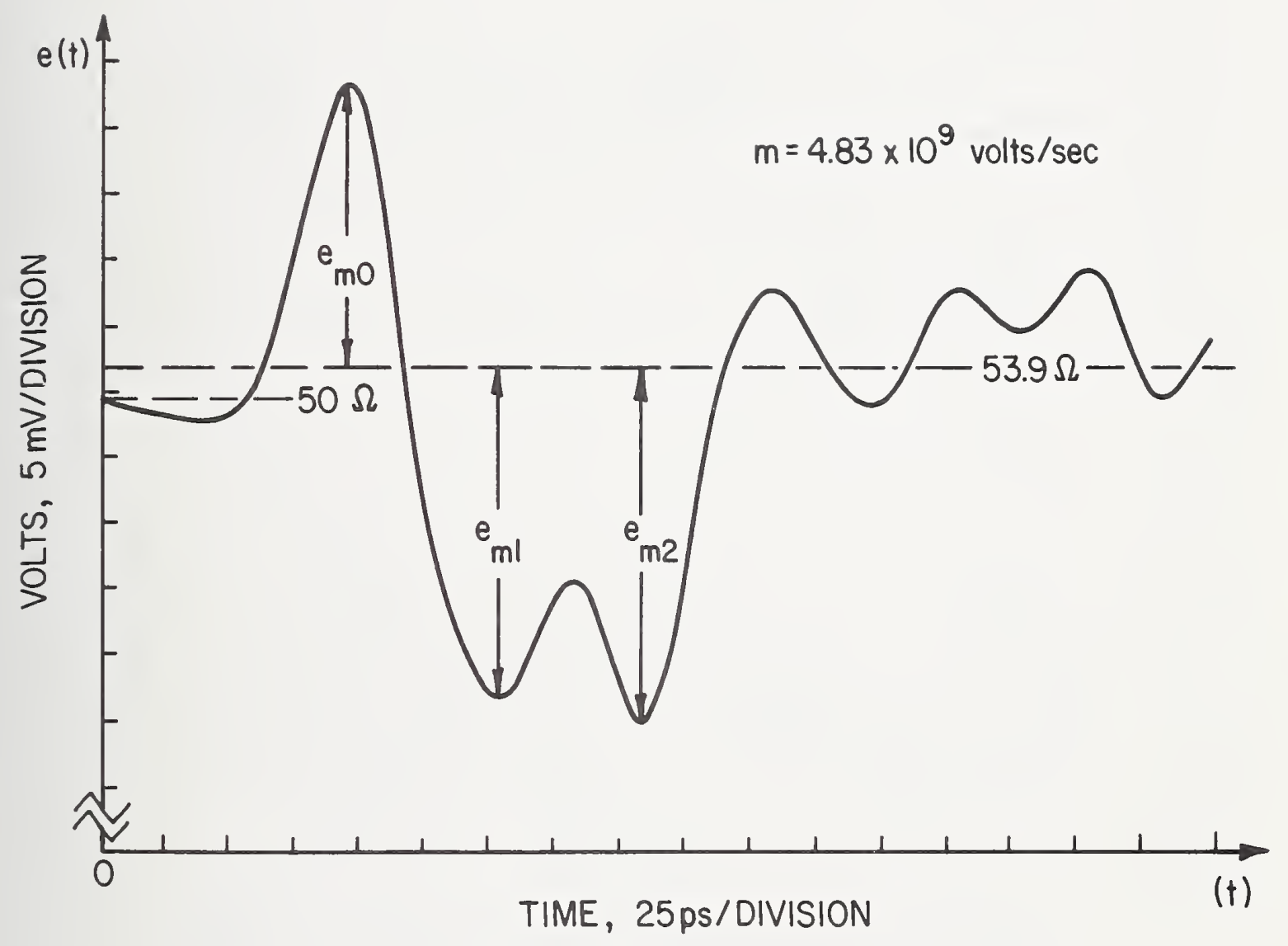

Figure 2.9 TDR signature of the connector used with the $1.55 \mathrm{~m}$ or $3.10 \mathrm{~m}$ line filled with 1.14-molal solution of 2-Heptanone in $\mathrm{n}$-Heptane. 

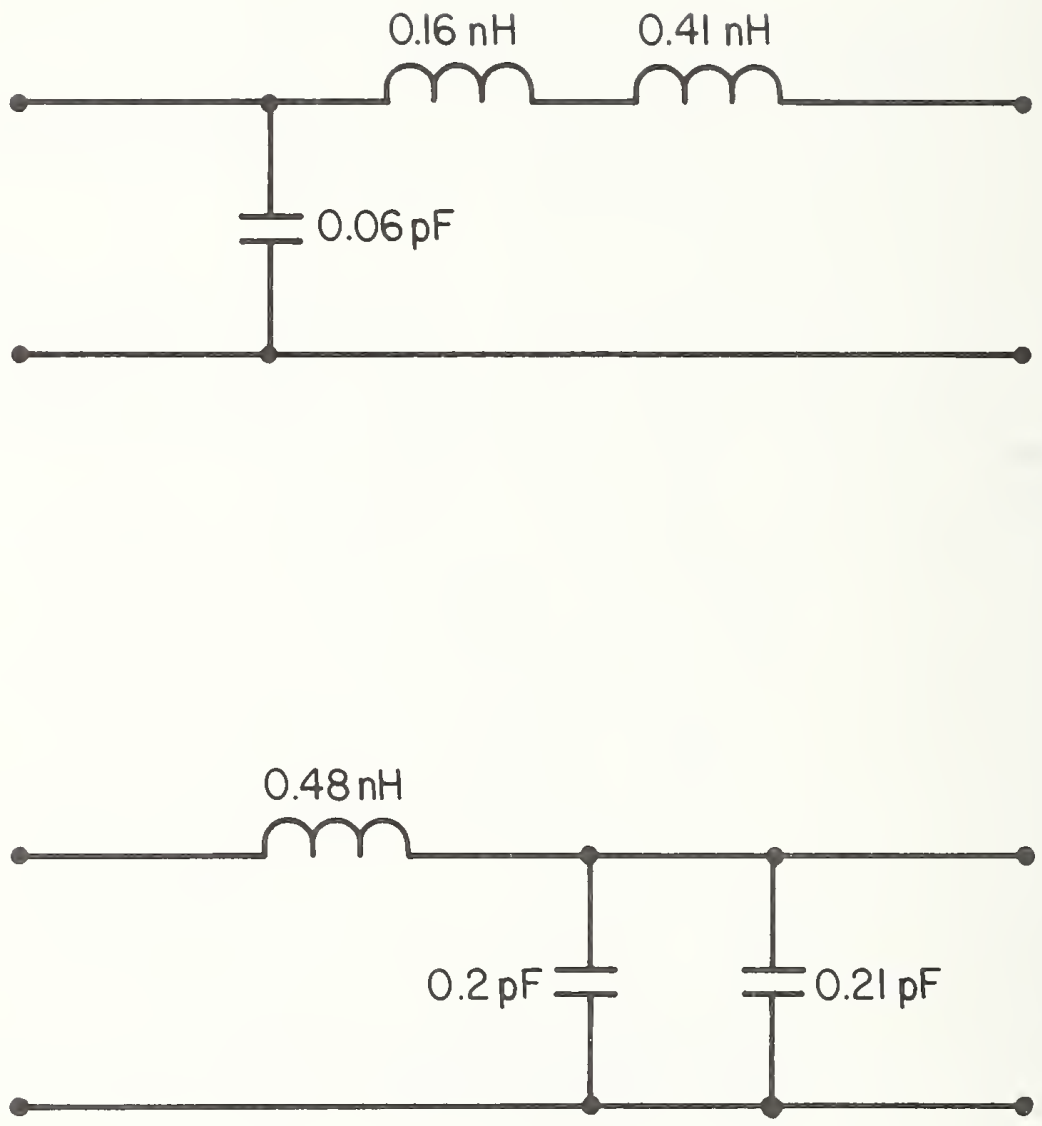

Figure 2.10 Lumped circuit equivalent networks for connector/adapters. 
STATIC DIELECTRIC CONSTANT TEMPERATURE COEFFICIENT, $a, \times 10^{3}$

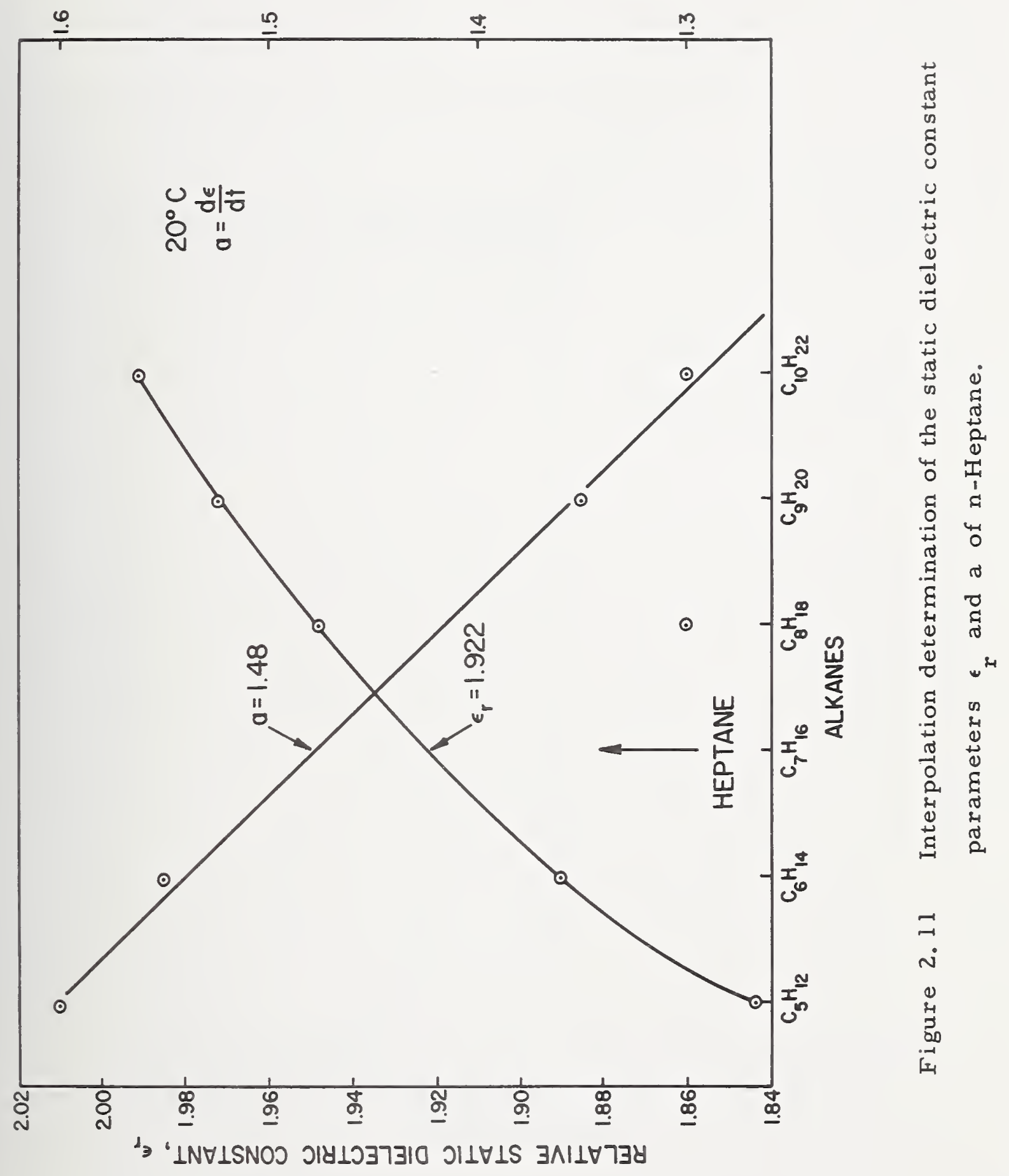




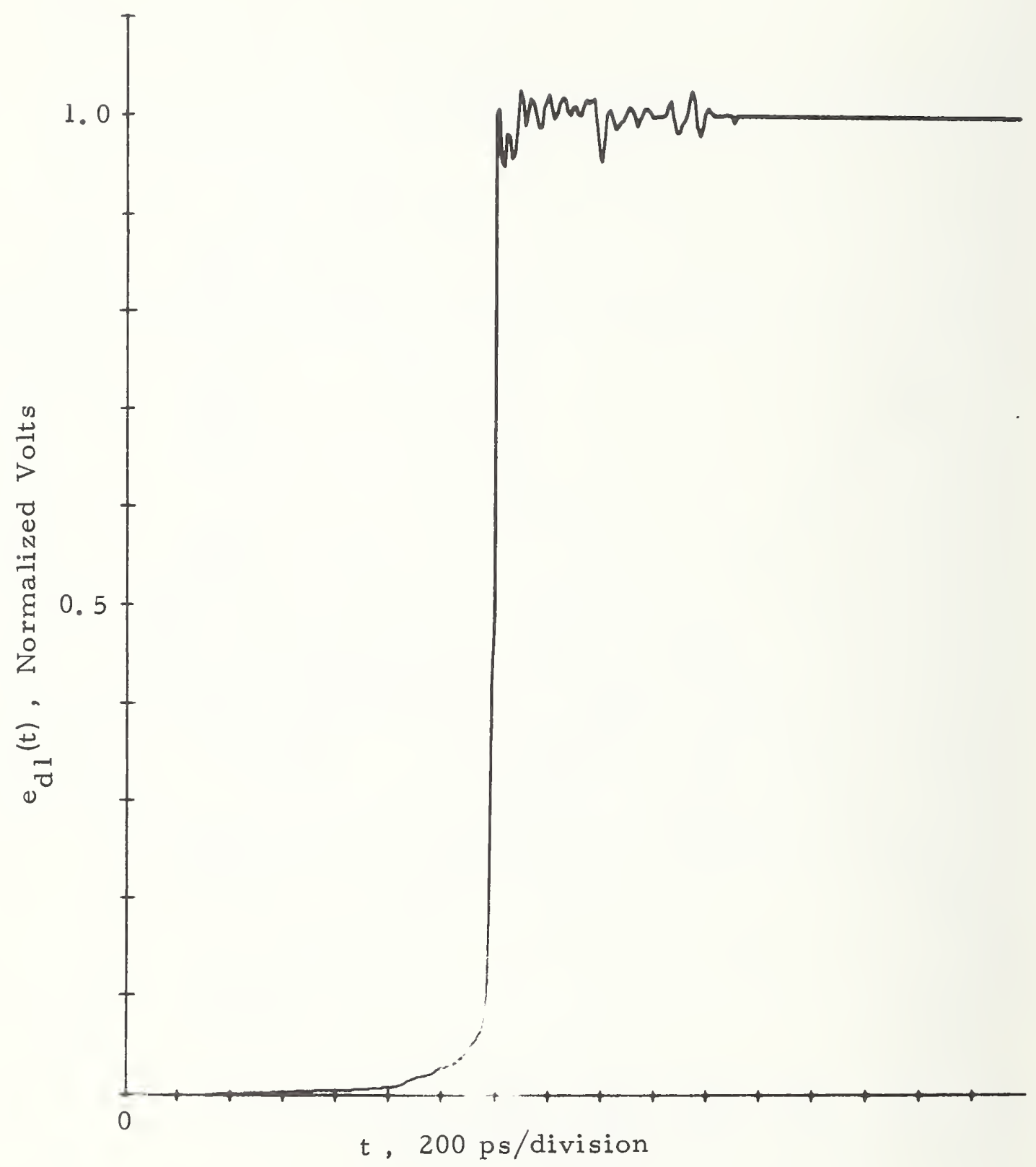

Figure 2.12 Digitized observed driving waveform $e_{d l}(t)$.

50 


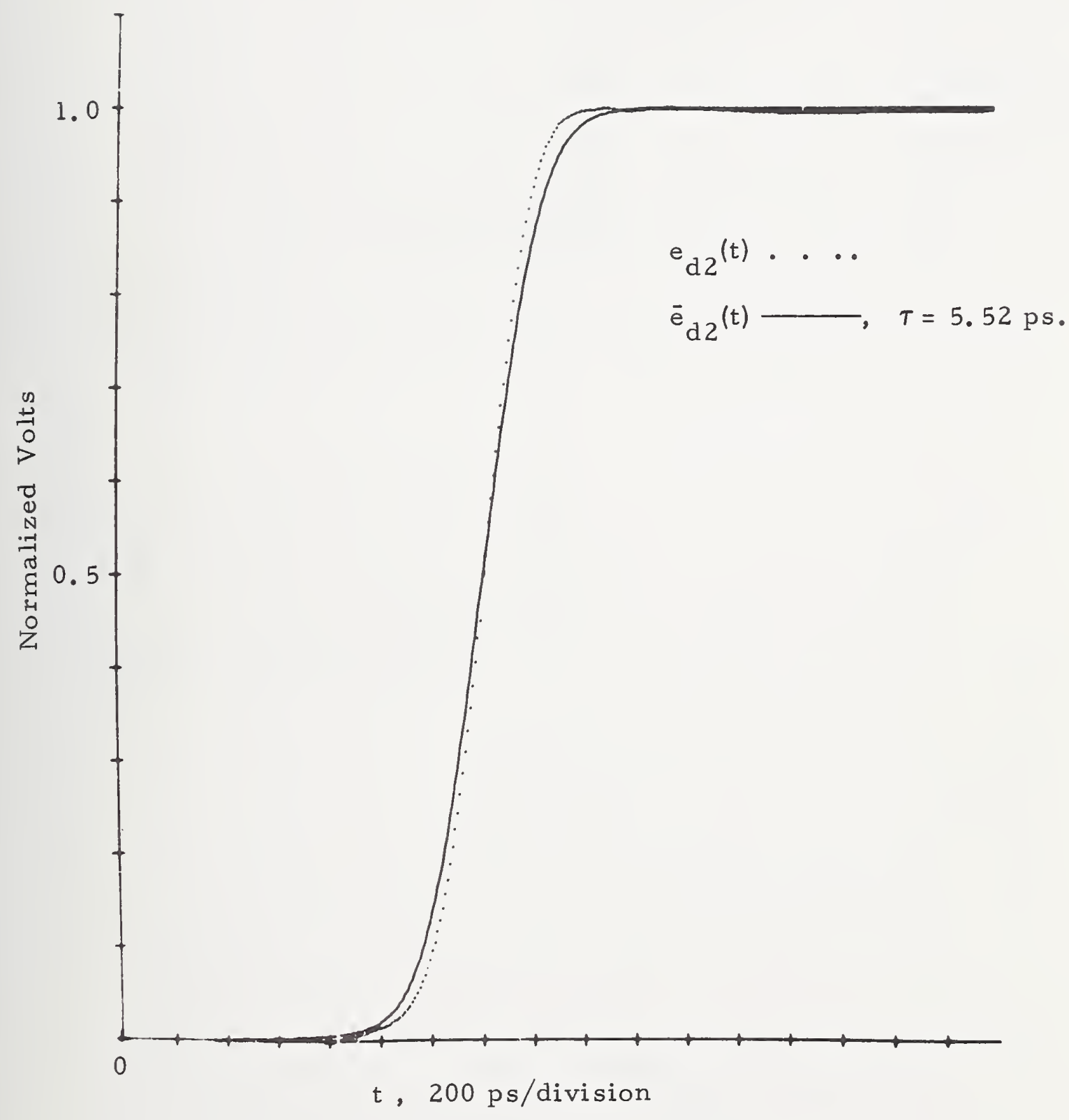

Figure 2.13 The observed insertion voltage $e_{d 2}(t)$ overlayed on computed insertion voltage $\bar{e}_{\mathrm{d} 2}(\mathrm{t})$ for $\tau=5.52$ ps. 


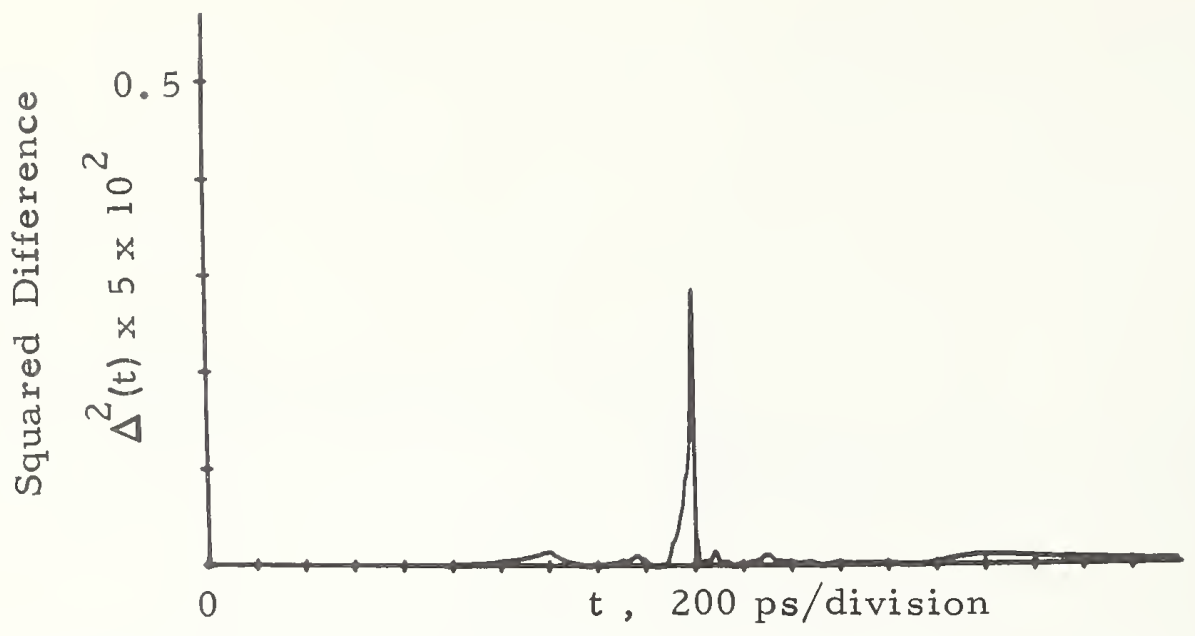

Figure 2.14A Squared difference between the observed insertion voltage $e_{d 2}(t)$ and the computed insertion voltage $\bar{e}_{d 2}(t)$.

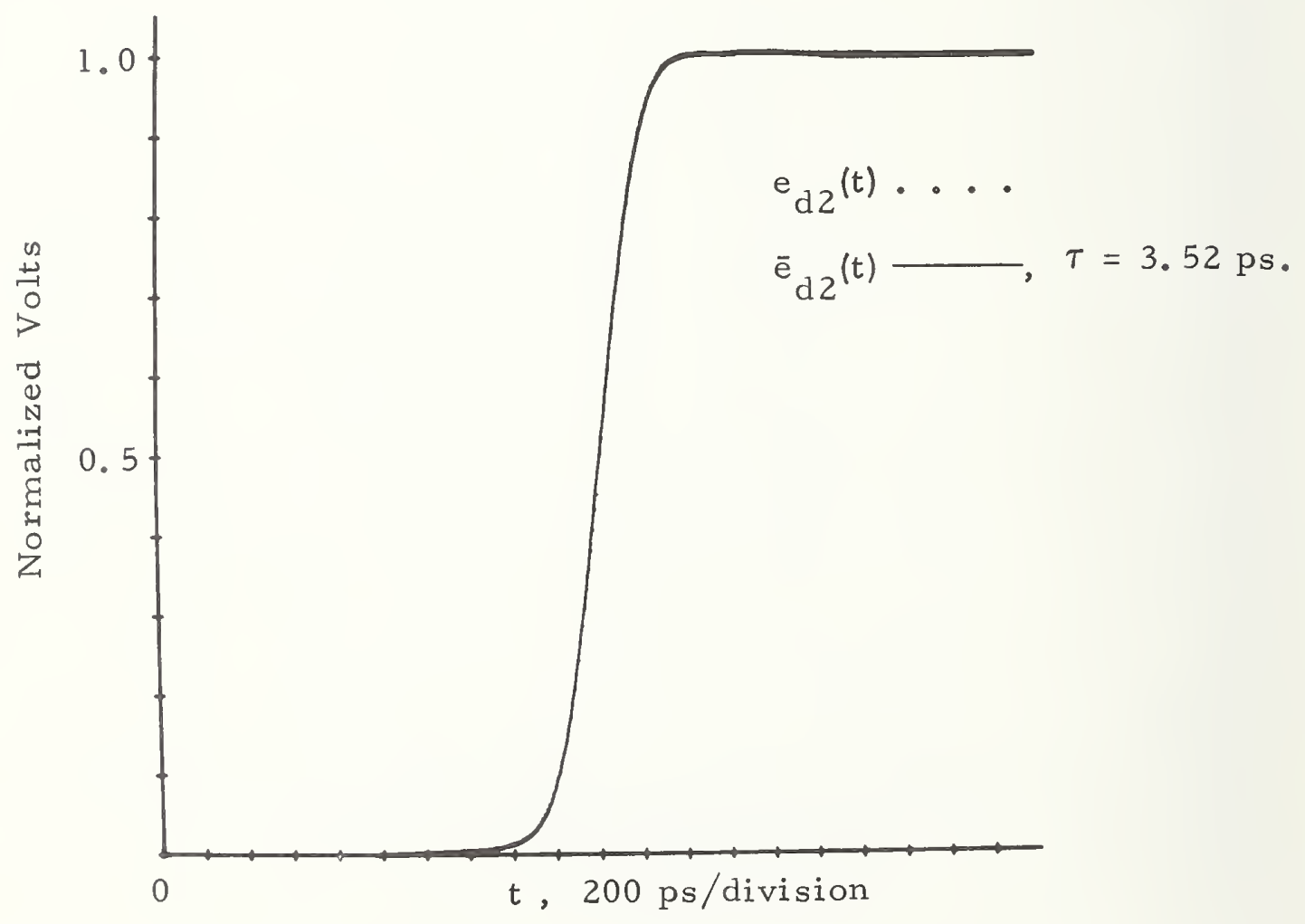

Figure 2.14B The observed insertion voltage $e_{d 2}(t)$ overlayed on the computed insertion voltage $\overline{\mathrm{e}}_{\mathrm{d} 2}(\mathrm{t})$ for $\tau=3.52 \mathrm{ps}$. 


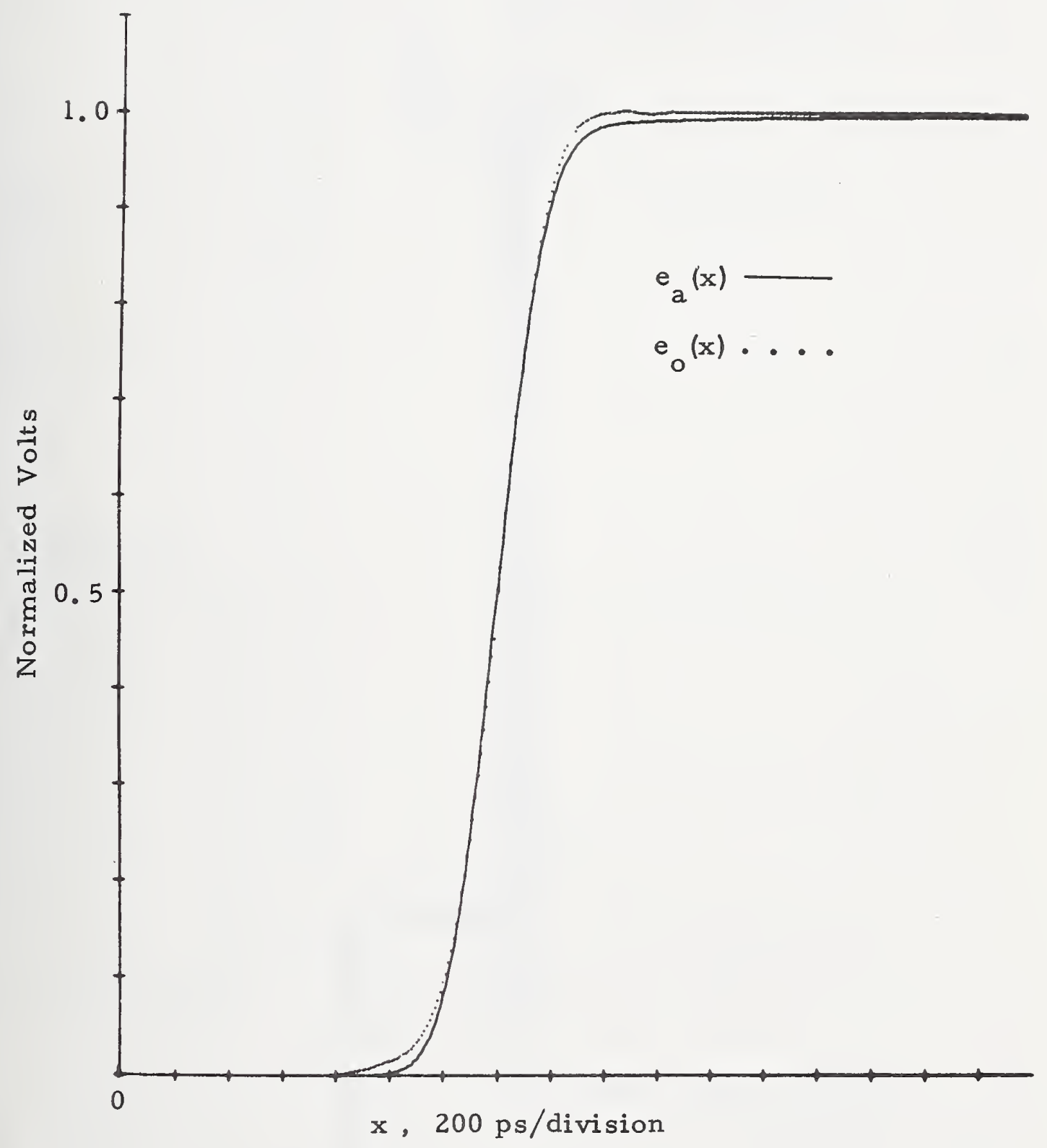

Figure 2.15 The generator output voltage $e_{o}(x)$ overlayed on the defined available waveform $e_{a}(x)$. 


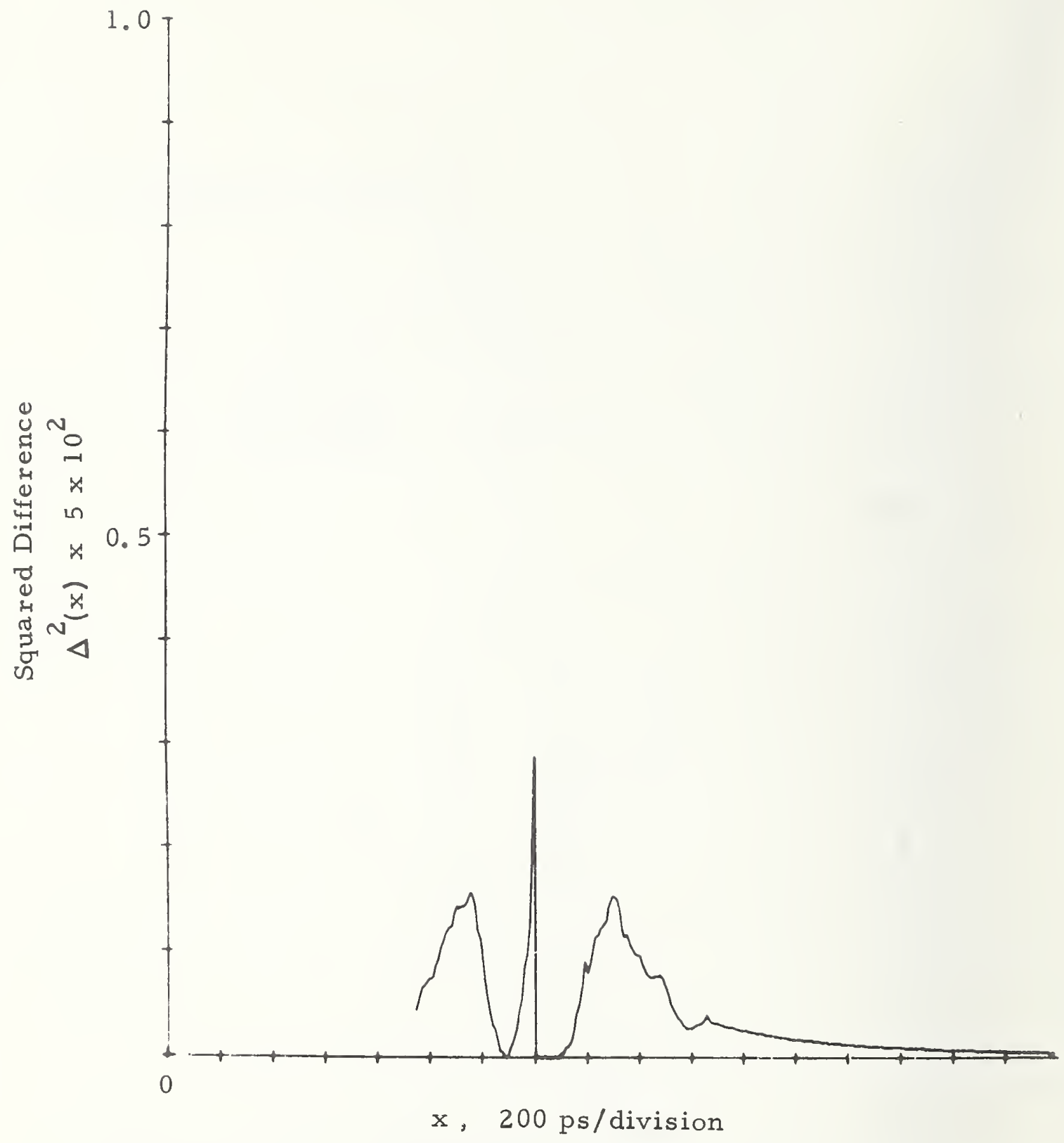

Figure 2.16 The squared difference of the output voltage $e_{0}(x)$ compared to the defined available waveform $e_{a}(x)$ for the waveforms shown in figure 2.15. 


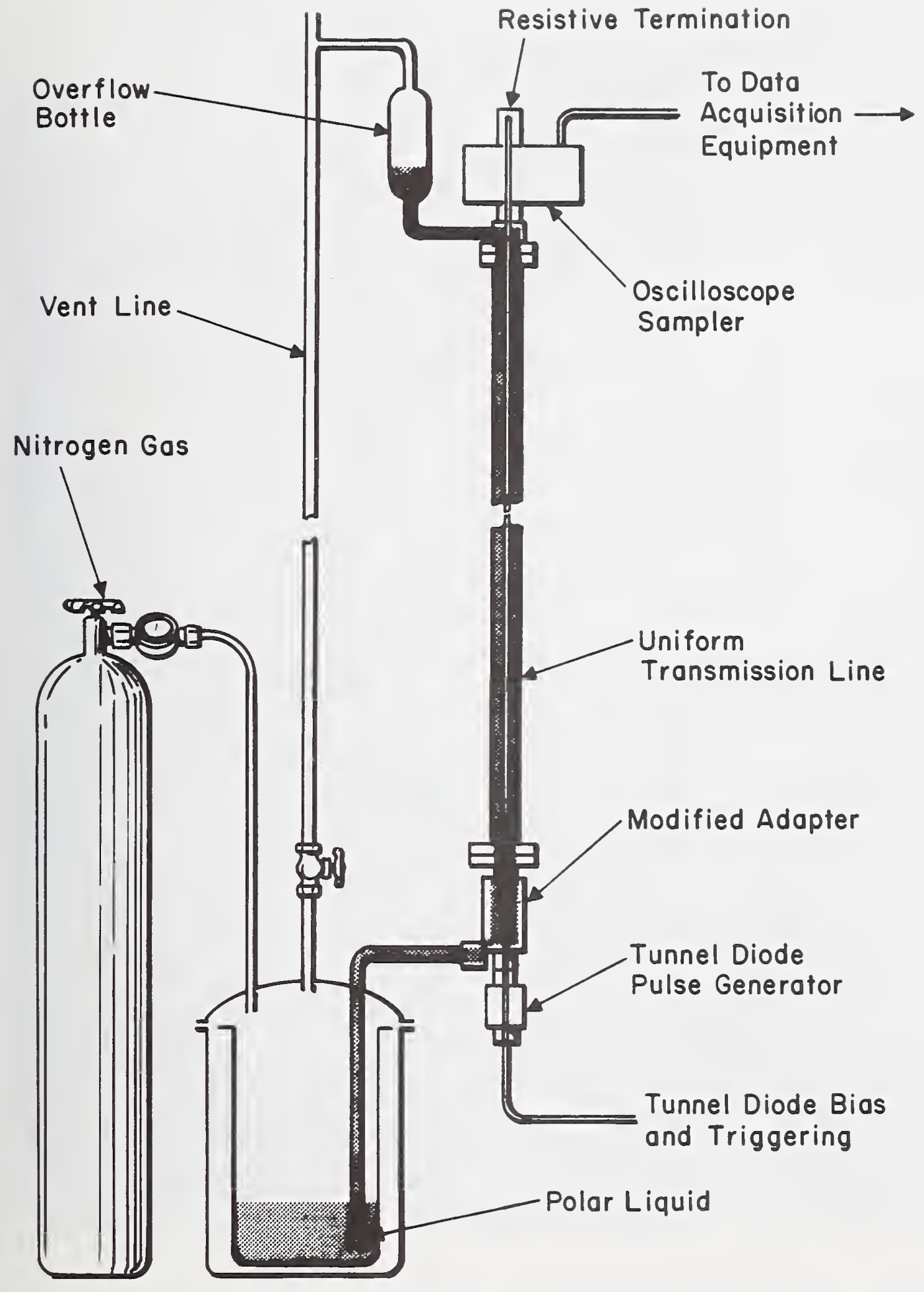

Figure 3.1 Liquid-dielectric filling system. 


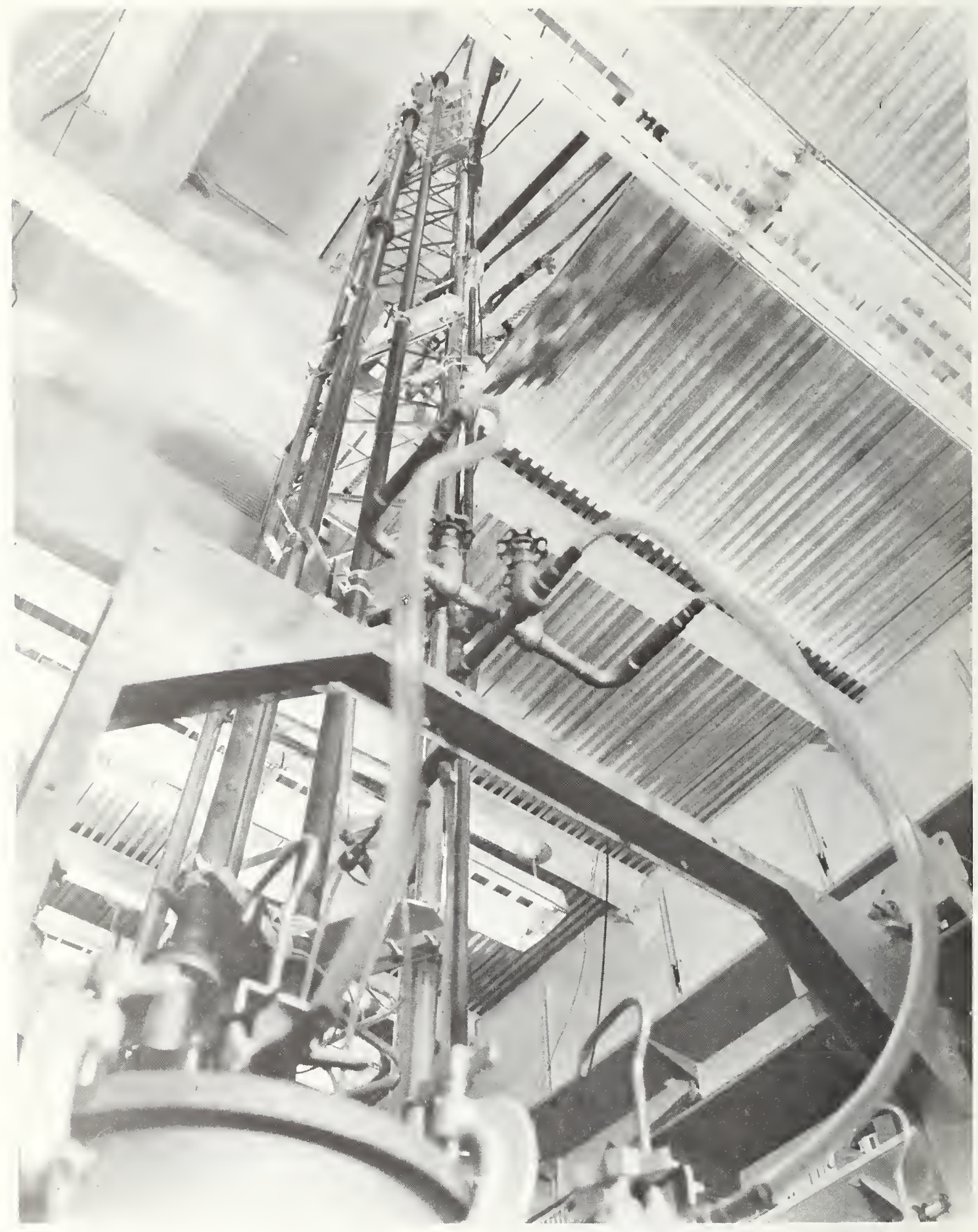

Figure 3.2 A view of the liquid-filled transmission lines installed on the supporting tower. 


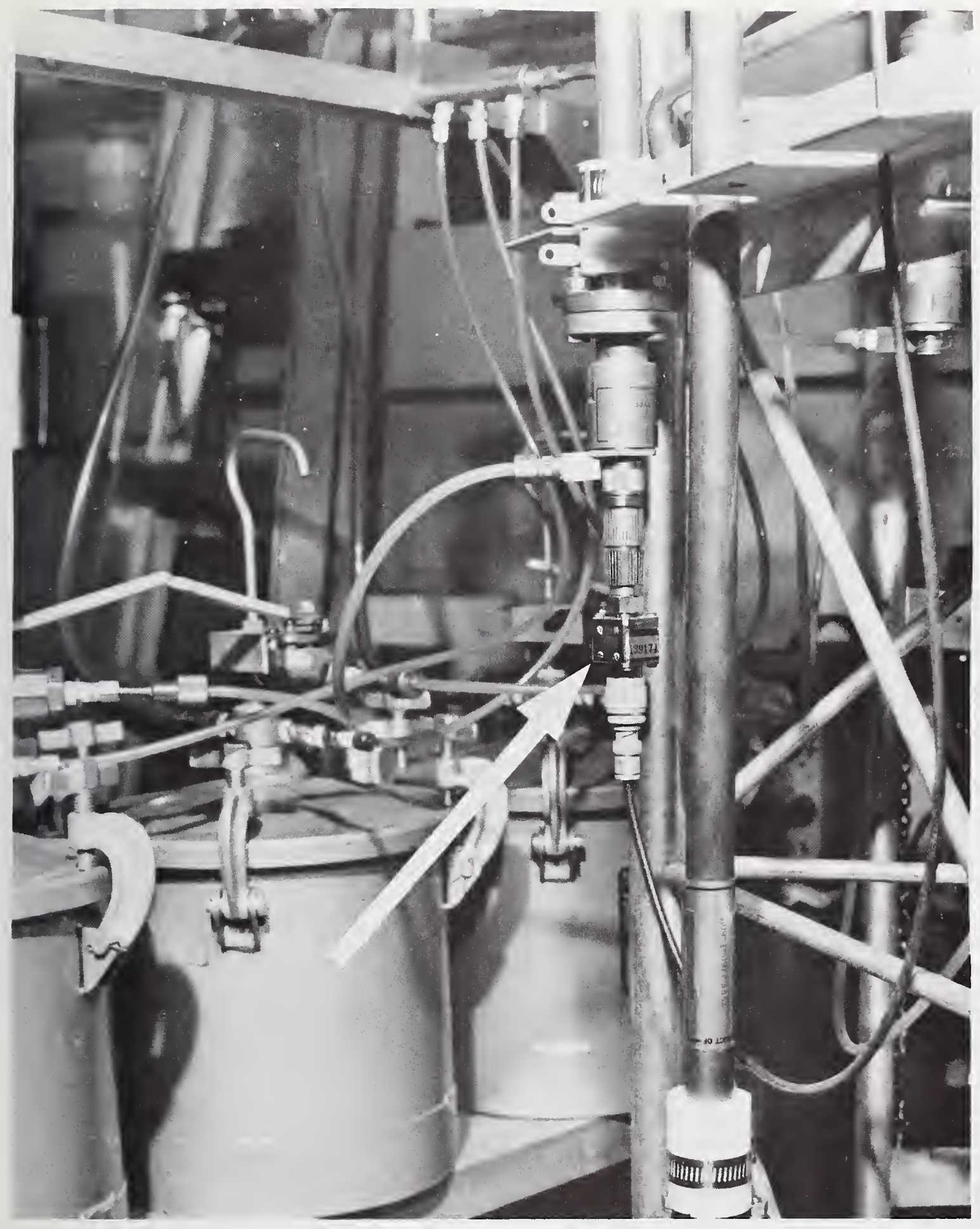

Figure 3.3 The tunnel-diode mount attached to the lower end of a liquid-filled transmission line. 


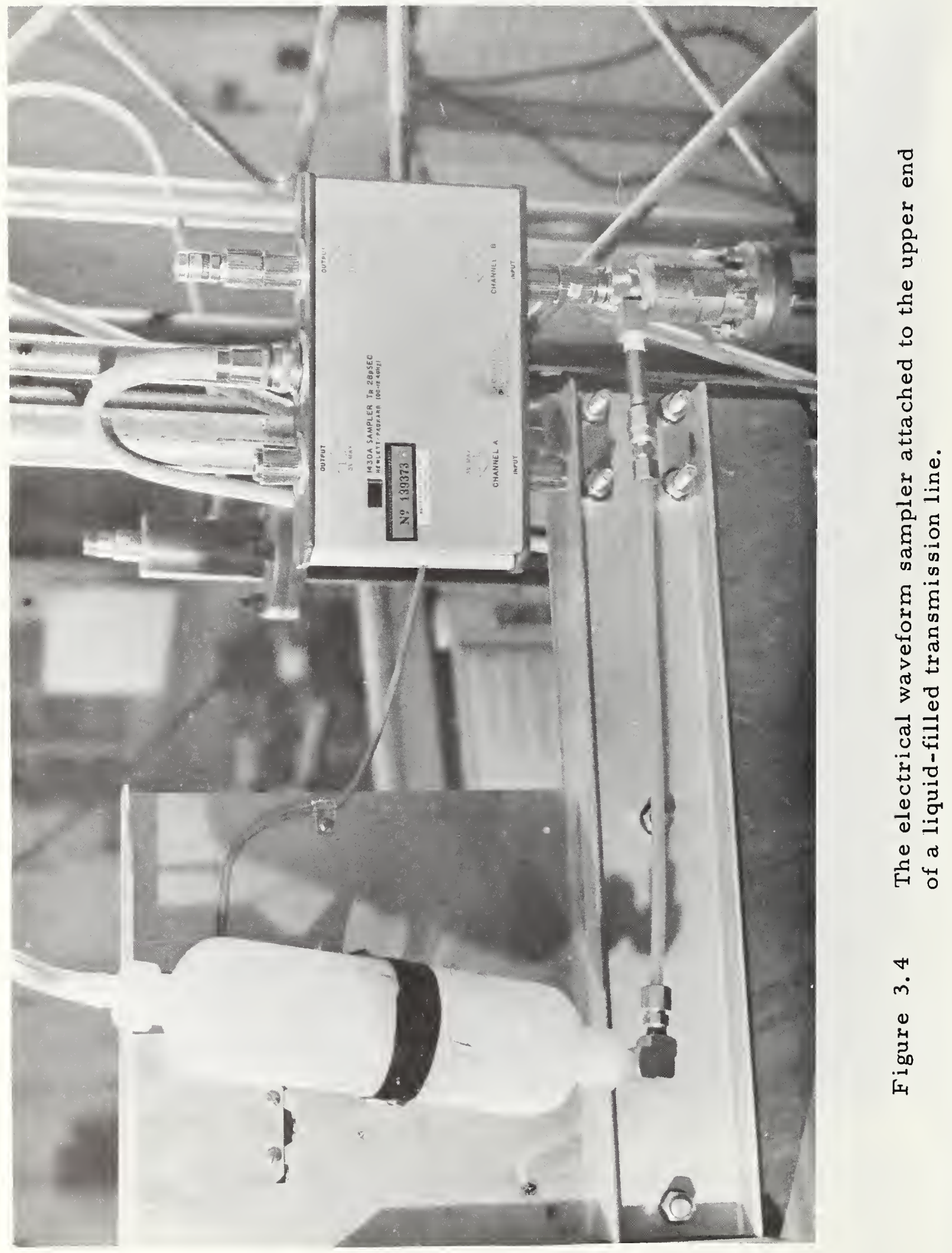




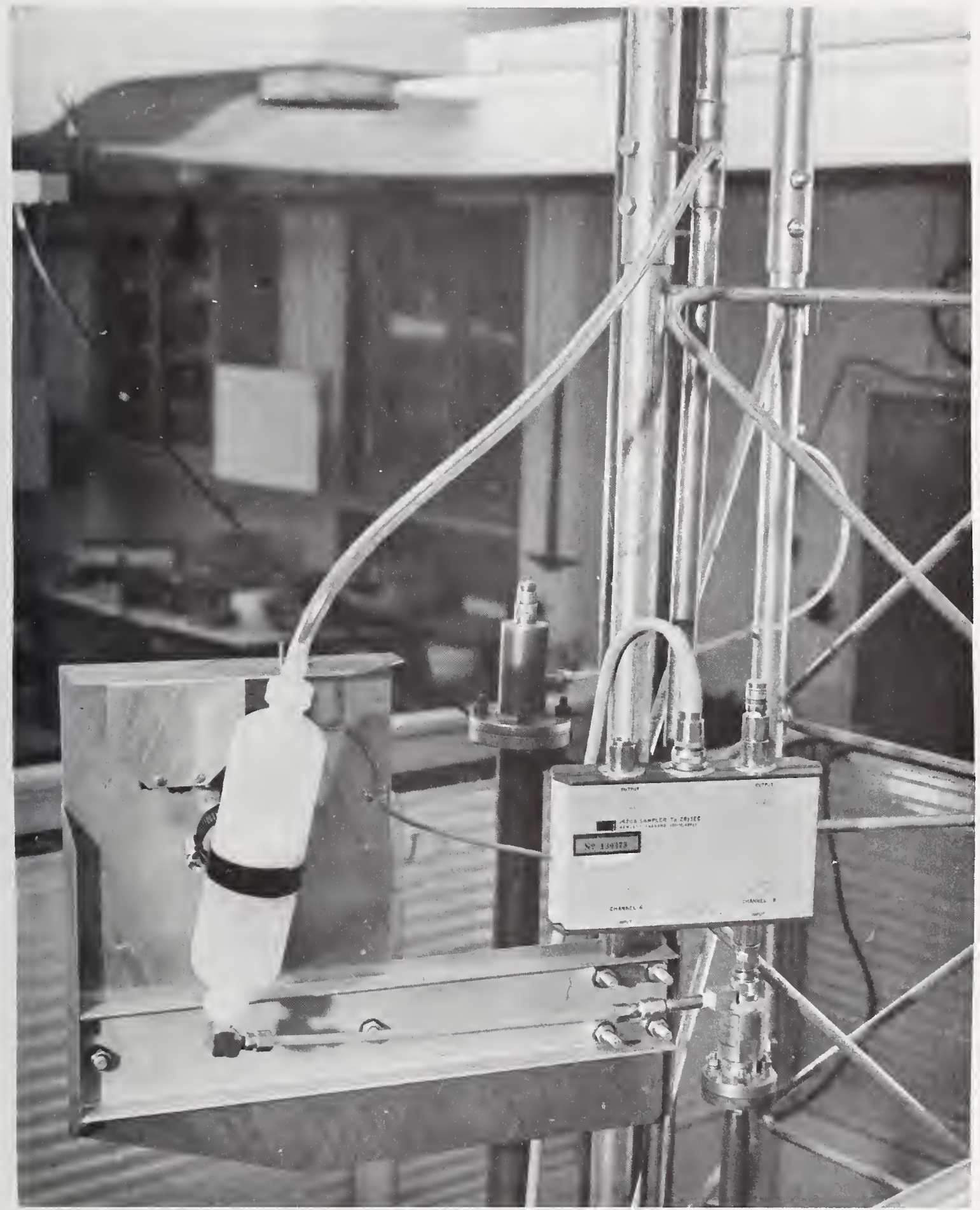

Figure 3.5 Overflow venting. 


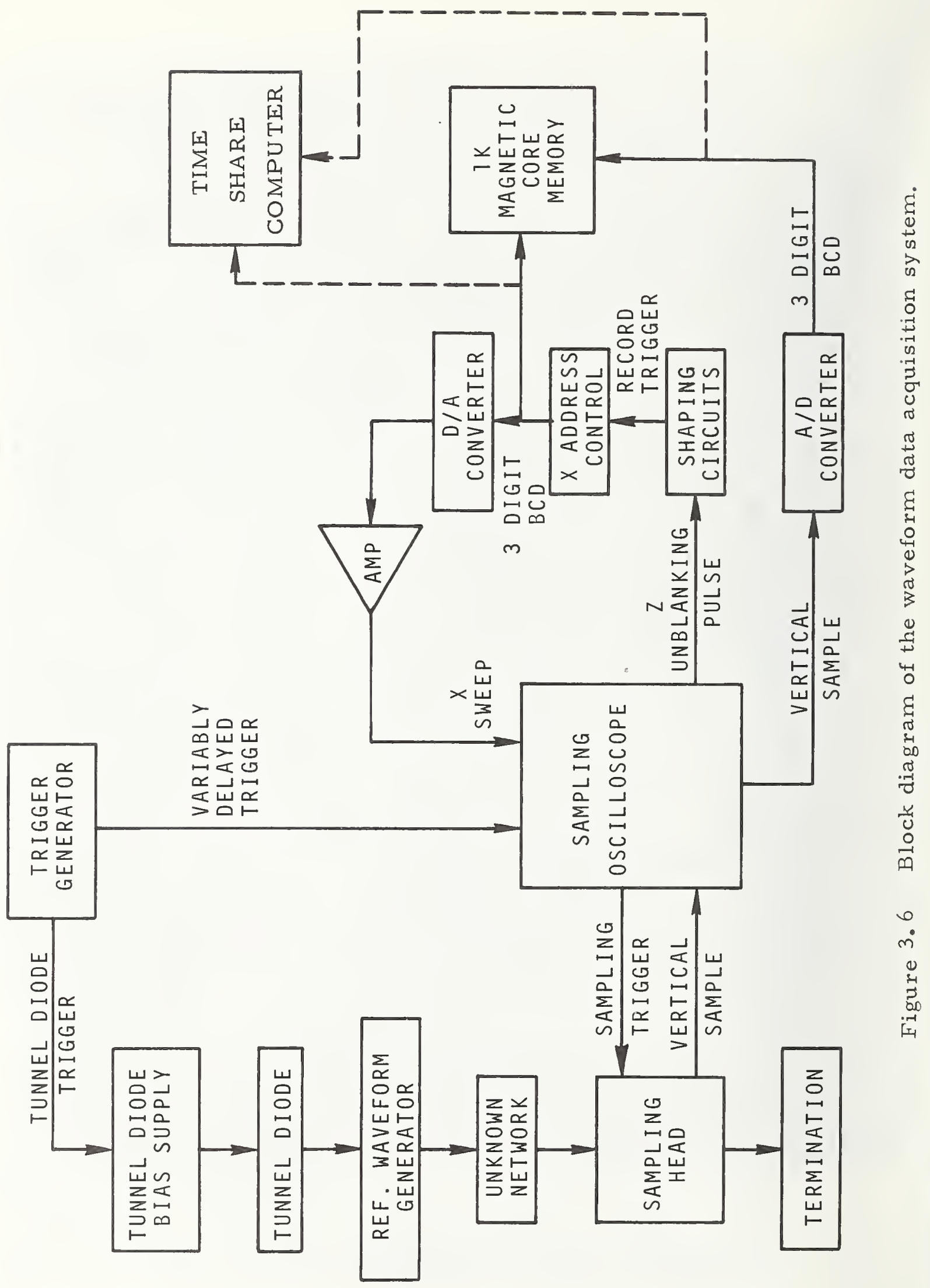


APPENDIX A - 1

TIME DOMAIN REFLECTOMETRY

\section{Fundamental Equations}

Consider the circuit shown in Fig. A-l. Here, $E_{g}(s)$ and $R_{01}$ represent a lossless transmission line of characteristic impedance $R_{01}$ being driven by a bandlimited generator $E_{g}(s)$ and terminated in an impedance $Z(s)$. The TDR signature is the voltage $E(s)$. The fundamental TDR equations are

$$
\begin{aligned}
& E(s)=E_{g}(s)-I(s) R_{01} \\
& E(s)=E_{i}(s)+E_{r}(s)
\end{aligned}
$$

where $E_{i}(s)$ and $E_{r}(s)$ are the incident and reflected components of $E(s)$. Equating $(A-1.1)$ to $(A-1.2)$ obtains

$$
E_{r}(s)=E_{g}(s)-E_{i}(s)-I(s) R_{01}
$$

Since the incident voltage $E_{i}(s)$ is the voltage when $Z(s)=R_{01}$, i.e., $E_{r}(s)=0$, then

$$
E_{i}(s)=\frac{E_{g}(s)}{2}
$$

which when put into $(A-1.3)$ to eliminate $E_{g}(s)$ obtains

$$
E_{r}(s)=E_{i}(s)-I(s) R_{01} \text {. }
$$


The inverse Laplace transformation of (A-1.5) yields the time domain reflected voltage $e_{r}(t)$

$$
e_{r}(t)=e_{i}(t)-i(t) R_{01}
$$

Similarly, the total time domain voltage of the TDR signature follows from $(A-1.1)$.

$$
e(t)=e_{g}(t)-i(t) R_{01}
$$

$(A-1.6)$ is useful for interpreting the reflected voltage while (A-1.7) establishes the absolute levels of the TDR signature. The nature of $Z(s)$ determines the properties of $i(t)$ under the excitation of $e_{g}(t)$.

2. The excitation $e_{g}(t)$

For the cases analyzed here, $e_{g}(t)$ will be assumed to be a finite ramp voltage as shown in Fig. A-2, that is

$$
e_{g}(t)= \begin{cases}a t, & 0 \leq t \leq T \\ a T, & T \leq t \leq \infty\end{cases}
$$


3. The current for an Inductive $Z(s)$

Consider Fig. A-3 which shows the TDR equivalent circuit for a series inductive discontinuity $\mathrm{sL}$ between two uniform lossless transmission lines of characteristic impedances $R_{01}$ and $R_{02}$, respectively. The current $I(s)$ is given by

$$
I(s)=\frac{E_{g}(s)}{R_{O T}+s L}
$$

where

$$
R_{0 T}=R_{01}+R_{02}
$$

Now

$$
e_{g}(t)=a t u(t)-a(t-T) u(t-T)
$$

where $u(t)$ is the unit step function. The Laplace transform of $(A-1.10) E_{g}(s)$ is given by

$$
\mathcal{L} e_{g}(t)=E_{g}(s)=\frac{a}{s^{2}}-e^{-s T} \frac{a}{s^{2}}
$$

therefore, 


$$
\begin{aligned}
I(s) & =I_{1}(s)+I_{2}(s) \\
& =\frac{a}{s^{2}} \frac{1}{\left(R_{0 T}+s L\right)}-e^{-s T} \frac{a}{s^{2}\left(R_{0 T}+s L\right)}
\end{aligned}
$$

In the time domain we have

$$
\begin{aligned}
i_{1}(t) & =s^{-1} I_{1}(s) \\
& =\frac{1}{2 \pi j} \int_{\gamma-j \infty}^{s t} e^{s t} I_{1}(s) d s \\
& =\frac{a}{L} \sum \text { Residues of } \frac{e^{s t}}{s^{2}\left(s+s_{0}\right)}
\end{aligned}
$$

where $s_{0}=R_{0 T} / L$. Continuing

$$
\begin{aligned}
\dot{i}_{1}(t) & =\frac{a}{L}\left[\left\{\frac{d}{d s}\left[\frac{e^{s t}}{\left(s+s_{0}\right)}\right]\right\}_{s=0}+\left[\frac{e^{s t}}{s^{2}}\right]_{i=s_{0}}\right] \\
& =\left\{\frac{a t}{R_{0 T}}-\frac{a L}{R_{0 T}^{2}}\left[1-e^{\left.\left.-t\left(\frac{R_{0 T}}{L}\right)\right]\right\} u(t)}\right.\right.
\end{aligned}
$$

Since

$$
I_{2}(s)=e^{-s T} I_{1}(s)
$$


$i_{2}(t)$ is equal to $-i_{1}(t)$ delayed $T$ seconds in time and multiplied by the delayed unit step function $u(t-T)$. Consequently, we have

$$
i(t)=\left[\frac{a t}{R_{0 T}}-\frac{a L}{R_{0 T}^{2}}\left(1-e^{\frac{-R_{0 T}}{L} t}\right) ; 0 \leq t \leq T .\right.
$$

and

$$
\begin{gathered}
i(t)=\left[\frac{a t}{R_{0 T}}-\frac{a L}{R_{0 T}^{2}}\left(1-e^{-\frac{R_{0 T}}{L} t}\right)\right]-\left[\frac{a(t-T)}{R_{0 T}}-\frac{a L}{R_{0 T}^{2}}\left(1-e^{-\frac{R}{L}(t-T)}\right)\right] \\
T \leq t \leq \infty
\end{gathered}
$$

From $(A-1.14)$ or $(A-1.15)$ for $t=T$, we obtain

$$
i(T)=\frac{a T}{R_{0 T}}-\frac{a L}{R_{0 T}^{2}}\left(1-e^{-\frac{R_{0 T}}{L} T}\right)(A-1.16)
$$

Upon combining terms and rearranging $(A-1.15)$ can be written in terms of $(A-1.16)$,

$$
i \leq t \leq \infty)=\frac{a T}{R_{0 T}}+\left[i(T)-\frac{a T}{R_{0 T}}\right] e^{-\frac{R_{0 T}}{L}(t-T)}
$$


4. The Reflected Voltage for an Inductive $\mathrm{Z}(\mathrm{s})$

The reflected voltage is given by putting $(A-1,14)$ and $(A-1,17)$ into $(A-1.6)$ :

$$
\underset{0 \leq t \leq T}{\left.e_{r}(t)\right]}=\frac{a t}{2}\left(\frac{R_{02}-R_{01}}{R_{0 T}}\right)+\frac{a L R_{01}}{R_{0 T}^{2}}\left(1-e^{-\frac{R_{0 T}}{L} t}\right)
$$

$$
\underset{T<t}{\left.e_{r}(t)\right]}=\frac{a T}{2}\left(\frac{R_{02}-R_{01}}{R_{0 T}}\right)-R_{01}\left(i(T)-\frac{a T}{R_{0 T}}\right) e^{\frac{-R_{0 T}}{L}(t-T)} ;
$$

Fig. A-4 qualitatively illustrates (A-1.18) and (A-1.19).

The reflected voltage can be normalized relative to the incident voltage steady state level, $\mathrm{E}_{0}$,

$$
E_{0}=\frac{a T}{2}
$$

Cons equently,

$$
a=\frac{2 E_{0}}{T}
$$

Upon replacing "a" by $(A-1.21)$ in $(A-1.18)$ and $(A-1.19)$ and then writing $e_{r}(t) / E_{0}$ as $R(t)$ obtains 


$$
\begin{aligned}
& \underset{R(t)]}{R \leq t \leq T}=\frac{t}{T}\left(\frac{R_{02}-R_{01}}{R_{0 T}}\right)+\frac{2 L R_{01}}{T R_{0 T}^{2}}\left(1-e^{-\frac{R_{0 T}}{L} t}\right) ; 0 \leq t \leq T \\
& \underset{T(t)]}{T<t<\infty}=\left(\frac{R_{02}-R_{0 I}}{R_{0 T}}\right)-R_{01}\left[\frac{i(T)}{E_{0}}-\frac{2}{R_{0 T}}\right] e^{-\frac{R_{0 T}}{L}(t-T)}
\end{aligned}
$$

For $t \rightarrow \infty, R(\infty)$ approaches the dc value of the reflection coefficient $\rho(0)$.

$$
R(\infty)=\rho(0)=\frac{R_{02}-R_{01}}{R_{02}+R_{01}}
$$

Recall that $R(t),(A-1.22)$ and $(A-1.23)$, is the reflected voltage normalized to the steady state value of the incident voltage $e_{i}(\infty)=E_{0}$. This normalized reflected voltage $R(t)$ is not equal to the impulse response of the reflection coefficient. Since,

$$
\hat{\rho}(t)=\Sigma^{-1} \rho(s)=\Sigma^{-1} \frac{Z(s)-R_{01}}{Z(s)+R_{01}}
$$

it is clear that

$$
\hat{\rho}(t) \neq R(t)
$$

except in the limit as $t$ approaches infinity.

Therefore, the designation of the scale for $R(t)$ in terms of resistive reflection $\rho$ units should not be interpreted to mean that $R(t)=\rho(t)$. The basis for using resistive reflection $\rho$-units stems from (A-1.24) which is a dc relationship. 
5. The Calculation of L from $e_{r}(t)$

Consideration of $(A-1.18)$ and $(A-1.19)$ leads to the conclusion that $L$ can be determined from $e_{r}(t)$ in either of two ways:

(a) By measuring the peak value of $\mathrm{e}_{r}(t)$ which occurs for $t=T$ and computing $L$ from $(A-1.18)$ with $t=T$.

(b) By measuring the time required for an $\mathrm{e}^{-1}$ magnitude change in the exponential trailing edge waveform of (A-1.19) and computing $L$ from the exponential time constant $\tau=L / R_{0 T}$.

For small reflections, i. e., small values of $L$ and subsequent small peak values of $e_{r}(t)$, the reflected voltage peak value is more visually discernable in wide-band sampling system noise than is the exponential decay. Consequently, for visual measurements the method of (a) is more effective. On the other hand, for systems in which signal processing methods are applied to the sampled-data analog signal, the method of (b) could be more appropriate.

We now turn our attention to the determination of $L$ from peak reflected voltage measurements as described in (a). Consider Fig. A-4 which shows the total voltage $e(t)=e_{r}(t)+e_{i}(t)$. Since the zero level of the reflected voltage $e_{r}(t)$ is the steady state value of the incident voltage, measurement of the difference between the peak value of the total voltage waveform and the steady state value of the incident waveform yields the peak value of the reflected voltage. Such a measurement is easily accomplished with a sampling oscilloscope by offsetting the steady state incident voltage level to the display zero 
level and using the high gain of the sampled-data amplifiers to enlarge the reflected waveform. The maximum reflected voltage occurs for $\mathrm{t}=\mathrm{T} .{ }^{*}$ From $(\mathrm{A}-1.18)$ we have

$$
e_{r}(T)=\frac{a T}{2}\left(\frac{R_{02}-R_{01}}{R_{0 T}}\right)+\frac{a I R_{01}}{R_{0 T}^{2}}\left(1-e^{-\frac{R}{L} T}\right)(A-1.26)
$$

For small reflections, that is, for small values of $L$, the network bandwidth limitations would be such that $R_{0 T}>|s L|$; consequently, the exponent $\frac{R_{0 T}}{L} T$ would be very large, and exp $\left[-\frac{R_{0 T}}{L} T\right]$ would be essentially zero. Under such conditions, $e_{r}(T)$ is given by

$$
\bar{e}_{r}(T)=\frac{a T}{2}\left(\frac{R_{02}-R_{01}}{R_{0 T}}\right)+\frac{a L R_{01}}{R_{0 T}^{2}}
$$

Here $\bar{e}_{r}(T)$ denotes $e_{r}(T)$ when $\exp \left[-\frac{R_{0 T}}{L} T\right]$ is essentially zero. Upon solving for the inductance $L$ we obtain

$$
L=\frac{R_{0 T}^{2}}{a R_{01}} \bar{e}_{r}(T)-\frac{T R_{0 T}}{2 R_{01}}\left(R_{02}-R_{01}\right)
$$

When $R_{02}=R_{01}, \quad R_{0 T}=2 R_{01}$, and $(A-1.28)$ becomes

$$
L=\frac{4 R_{01}}{a} \bar{e}_{r}(T)
$$

* If one considered eq. A-1.18 to hold for all time and $R_{02}<R_{01}$, then the maximum value of $e_{r}$ would occur at $t_{\max }=-\frac{L}{R_{0 T}} \ln \left(\frac{R_{01}-R_{02}}{2 R_{01}}\right)$. If this $t_{\max }$ is sufficiently less than $T$, then the following equations and conclusions are no longer applicable. 
This result is identical to the band-limited case $\left(2 R_{01}>s L\right)$ results given by $[2,3]$; it can be seen to be identical by putting it into the form

$$
\begin{aligned}
& L=\frac{2 R_{01}}{\left(\frac{a}{2}\right)} \bar{e}_{r}(T) \\
& L=\frac{\left[\text { maximum voltage referred to } e_{i}(\infty)\right]}{[\text { slope of the incident voltage ramp }]} \times 2 R_{0}
\end{aligned}
$$

The general result for $L$ when $R_{01} \neq R_{02}$ given by (A-1.28) requires that $\bar{e}_{r}(T)$ be measured from its inherent zero level which is absolutely equal to the steady state incident voltage $E_{0}=\frac{a T}{2}$. If the peak value of the voltage is measured from the steady state value of the reflected voltage $e_{r}(\infty)$, i.e., from the value given by $(A-1.19)$ with $t \rightarrow \infty$,

$$
e_{r}(\infty)=\frac{a T}{2}\left(\frac{R_{02}-R_{01}}{R_{0 T}}\right)
$$

then we have

$$
\bar{e}_{r}(T)-e_{r}(\infty)=\frac{a L R_{01}}{R_{0 T}^{2}} .
$$

Defining,

$$
\mathrm{e}_{\mathrm{m}}(\mathrm{T})=\left[\overline{\mathrm{e}}_{\mathrm{r}}(\mathrm{T})-\mathrm{e}_{\mathrm{r}}(\infty)\right]
$$


we can then write

$$
e_{m}(T)=\frac{a L R_{01}}{R_{0 T}^{2}}
$$

and

$$
L=\frac{R_{0 T}^{2}}{a R_{01}} e_{m}(T)
$$

or

$$
L=\frac{\left[\text { maximum voltage referred to } \mathrm{e}_{\mathrm{r}}(\infty)\right]}{[\text { slope of the incident voltage ramp }]} \frac{\mathrm{R}_{0 \mathrm{~T}}^{2}}{2 \mathrm{R}_{01}}
$$

This last result is the most practical one. Comparison of (A-1.32) with $(A-1.28)$ shows that to use $(A-1.28)$ one would in fact have to first determine $e_{r}(\infty)$ to compute $L$. Consequently, use of $(A-1.32)$, i. e., (A-1.35) includes $\mathrm{e}_{\mathrm{r}}(\infty)$ automatically.

\section{Dual Equations for Capacitive Loads}

Capacitive reflections are conveniently viewed in terms of admittance parameters and the node voltage $E(s)$ due to a current generator, Fig. A-5.

Here, $I_{g}(s)$ and $G_{01}$ represent a lossless transmission line of characteristic admittance $G_{01}$ being driven by a bandlimited generator 
$I_{g}(s)$ and terminated in an admittance $Y(s)$. The fundamental TDR equations in terms of incident and reflected currents a re

$$
\begin{aligned}
& I(s)=I_{g}(s)-E(s) G_{01} \\
& I(s)=I_{i}(s)+I_{r}(s)
\end{aligned}
$$

Since the incident current $I_{i}(s)$ is the current when $Y(s)=G_{01}$, $\left(I_{r}(s)=0\right)$, then

$$
I_{i}(s)=\frac{I_{g}(s)}{2}
$$

which when put into $(A-1.36)$ to eliminate $I_{g}(s)$ yields

$$
I_{r}(s)=I_{i}(s)-E(s) G_{01} \text {. }
$$

The inverse Laplace transformation of (A-1.39) yields

$$
i_{r}(t)=i_{i}(t)-e(t) G_{o l}
$$

Similarly the total TDR time domain current follows from (A-1.36)

$$
i(t)=i_{g}(t)-e(t) G_{01}
$$

These are the dual equations to $(A-1.6)$ and $(A-1.7)$.

7. The Excitation $i_{g}(t)$

For the cases analyzed here, $i_{g}(t)$ will be assumed to be a finite ramp current as shown in Fig. A-6, that is, 


$$
i_{g}(t)-b t u(t)-b(t) u(t-T)
$$

where $u(t)$ is the unit step function.

\section{The Voltage for a Capacitive $\mathrm{y}(\mathrm{s})$.}

Consider Fig. A-7 which shows the TDR equivalent circuit for a shunt capacitive discontinuity $\mathrm{s}$ at the junction of two uniform lossless transmission lines of characteristic admittances $G_{01}$ and $G_{02}$, respectfully. The voltage $E(s)$ is given by

$$
E(s)=\frac{I_{g}(s)}{G_{O T}+s C}
$$

where

$$
G_{0 T}=G_{01}+G_{02} \text {. }
$$

Since $(A-1.42)$ and $(A-1.43)$ are the duals of $(A-1.10)$ and $(A-1.9)$, respectively, the voltage

$$
e(t)=s^{-1} \frac{I_{g}(s)}{G_{O T}+s C}
$$

is the dual of the current $i(t)$ given by $(A-1,14)$ and $(A-1.15)$. Consequently, we can immediately write down $e(t)$ by inspection of $(A-1.14)$ and $(A-1.15)$ through the dual relationships. Hence we have from $(A-1.14)$ and $(A-1.17)$

$$
e(t)=\left[\frac{b t}{G_{O T}}-\frac{b C}{G_{O T}^{2}}\left(1-e^{-\frac{G_{O T}}{C} t}\right)\right] ; 0 \leq t \leq T
$$


and

$$
e(t)=\frac{b T}{G_{O T}}+\left[e(T)-\frac{b T}{G_{O T}}\right] e_{T<t<\infty}^{\frac{-G_{0 T}}{C}(t-T)}
$$

9. The Reflected Voltage for a Capacitive Load

Recalling that the total voltage is equal to

$$
e(t)=e_{i}(t)+e_{r}(t)
$$

we can write

$$
e_{r}(t)=e(t)-e_{i}(t)
$$

Also, from Thevinin's theorem we have that

$$
E_{g}(s)=I_{g}(s) / G_{01}
$$

Therefore,

$$
\frac{a}{s^{2}}\left(1-e^{-s T}\right)=\frac{b / G_{01}}{s^{2}}\left(1-e^{-s T}\right)
$$

which yields

$$
b=a G_{01}
$$


Upon putting ( $A-1.48)$ into $(A-1.45)$ and $(A-1.46)$, and the results into (A-1.47) we obtain

$$
\begin{gathered}
e_{r}(t)=\frac{a G_{01} t}{G_{0 T}}-e_{i}(t)-\frac{a G_{01} C}{G_{0 T}^{2}}\left(1-e^{-\frac{G_{0 T}}{C}}\right) ; \\
0 \leq t \leq T
\end{gathered}
$$

and

$$
e_{r}(t)=\frac{a G_{01} T}{G_{0 T}}-e_{i}(t)+\left[\begin{array}{c}
e(T)-\frac{a G_{01} T}{G_{0 T}} \\
T \leq t \leq \infty,
\end{array}\right] e^{-\frac{G_{0 T}}{C}(t-T) ;}
$$

Since

$$
\begin{aligned}
& \left.e_{i}(t)\right]=\frac{a t}{2} ; \quad 0 \leq t \leq T \\
& 0 \leq t \leq T \\
& \left.e_{i}(t)\right]=\frac{a T}{2} ; \quad T \leq t \leq \infty \\
& T \leq t \leq \infty \\
& R_{p}=\frac{1}{G_{0 T}}=\frac{R_{01} R_{02}}{R_{01}+R_{02}} \\
& R_{01}=\frac{1}{G_{01}}
\end{aligned}
$$

We can write for $(A-1.49)$ and $(A-1.50)$

$e_{r}(t)=\frac{a t}{2}\left(\frac{R_{02}-R_{01}}{R_{01}+R_{02}}\right)-\frac{R_{p}^{2} a C}{R_{01}}\left(1-e^{-\frac{t}{R_{p} C}}\right) ; 0 \leq t \leq T$, 
and

$e_{r}(t)=\frac{a T}{2}\left(\frac{R_{02}-R_{01}}{R_{01}+R_{02}}\right)+\left[e(T)-\frac{a R_{02} T}{\left(R_{01}+R_{02}\right)}\right] e$

$e^{-\frac{1}{R_{p} C}(t-T)}$

$\mathrm{T} \leq \mathrm{t} \leq \infty$

$(A-1.52)$

Fig. A-8 qualitatively illustrates $(A-1.51)$ and $(A-1.52)$.

10. The Calculation of $C$ from $e_{r}(t)$

The comments given in Section 5 also apply here. One can use the exponential decay for $t>T$ or can use the peak value of $e_{r}(t)$. We now develop the formulae for small $C$ from the peak value of $e_{r}(t)$.

The peak value occurs at $t=T$; consequently we have from $(A-1.51)$ or $(A-1.52)$

$$
e_{r}(T)=\frac{a T}{2}\left(\frac{R_{02}-R_{01}}{R_{01}+R_{02}}\right)-\frac{R_{p}^{2} \text { a } C}{R_{01}}\left(1-e^{-\frac{T}{R_{p} C}}\right)
$$

We again invoke a bandwidth limitation such that for small values of $c$, $G_{0 T}=\frac{1}{R_{P}}>|s C|$; consequently $\exp \left(-T / R_{P} C\right)$ is es sentially zero,

$$
\bar{e}_{r}(T)=\frac{a T}{2}\left(\frac{R_{02}-R_{01}}{R_{01}+R_{02}}\right)-\frac{R_{p}^{2} a C}{R_{01}}
$$

Upon referring the peak value of the total load voltage to the steady state value of the reflected voltage, we obtain 


$$
\begin{aligned}
& e_{m}(T)=\bar{e}_{r}(T)-e_{r}(\infty) \\
& e_{m}(T)=\frac{R_{p}^{2} a C}{R_{01}}
\end{aligned}
$$

and

$$
C=\frac{e_{m}(T) R_{01}}{a R_{p}^{2}}
$$

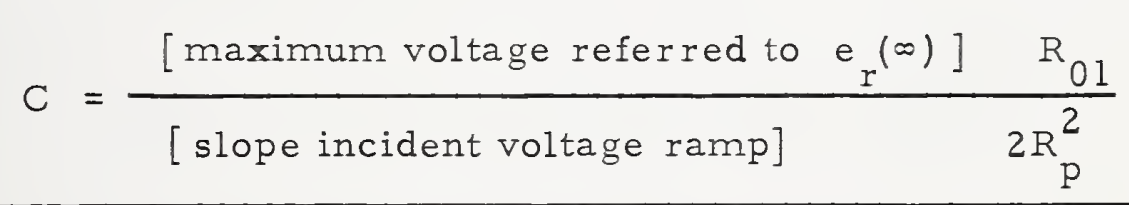

When $R_{01}=R_{02}, \quad R_{p}=\frac{R_{01}}{2}$ and

$$
C=\frac{\left[\text { maximum voltage referred to } e_{i}(\infty)\right.}{[\text { slope incident voltage ramp }]} \frac{2}{R_{01}}
$$

which is the usual formula $[2,3]$ for $C$ when $R_{01}=R_{02}$. 


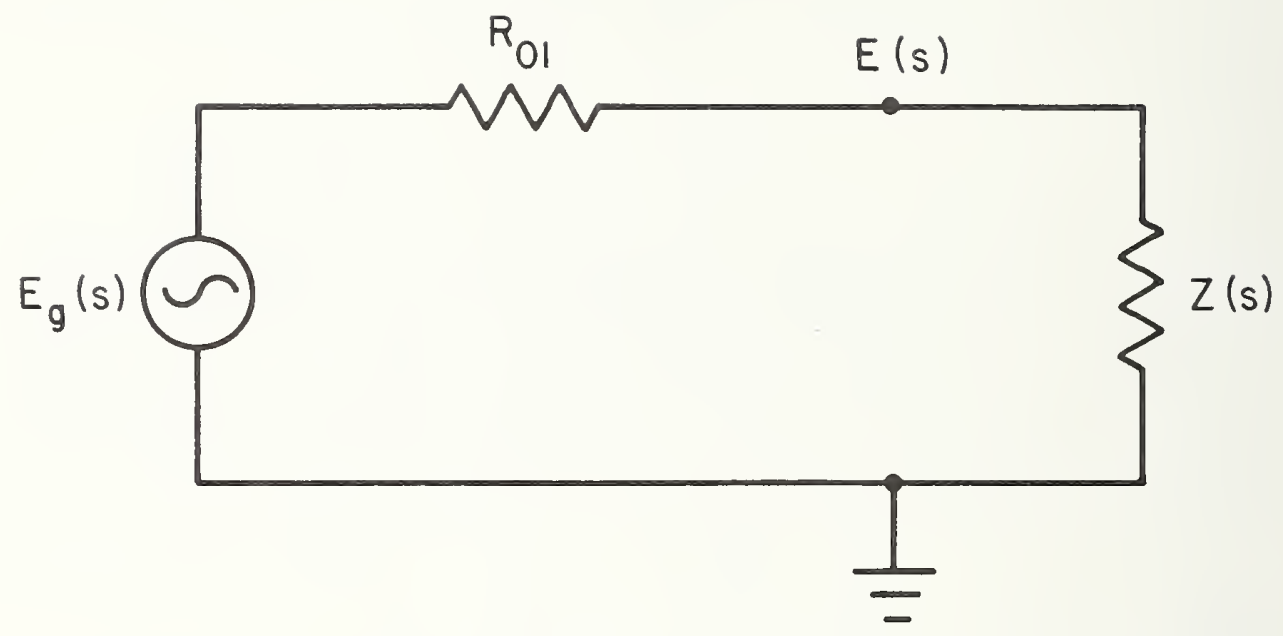

Figure A-1 The input equivalent circuit for a uniform lossless transmission line terminated in an impedance $Z(s)$ and driven by a bandlimited generator $\mathrm{E}_{\mathrm{g}}(\mathrm{s}) . \mathrm{R}_{01}$ is the transmission line characteristic impedance. 
<smiles>[H]</smiles> 


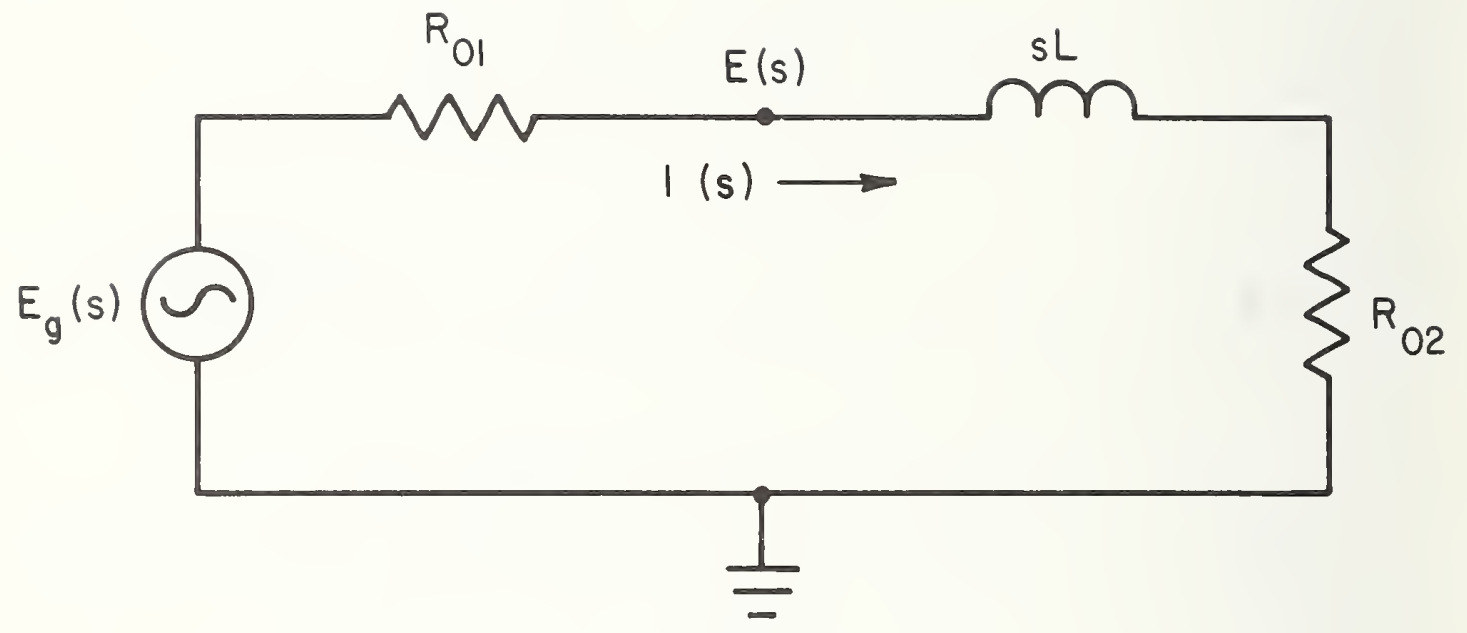

Figure A-3 The TDR equivalent circuit for a series inductive discontinuity $\mathrm{sL}$ between two uniform transmission lines of characteristic impedances $R_{01}$ and $R_{02}$ respectively. 

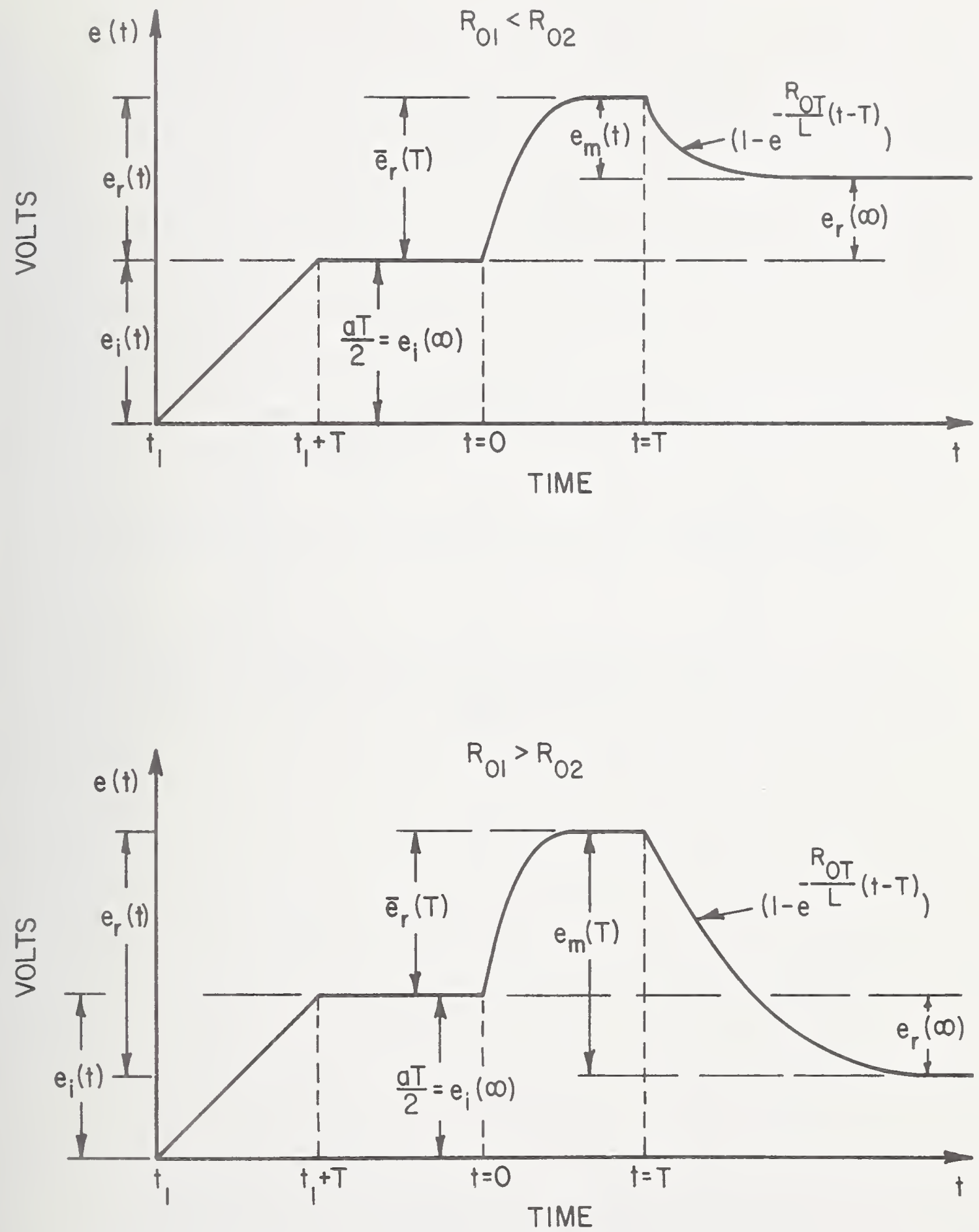

Figure A-4 Qualitative time domain responses for the total inductive load voltage. A delay line of time delay $t_{1} / 2$ and characteristic impedance $R_{01}$ is used to isolate in time the observation point and the discontinuity. 


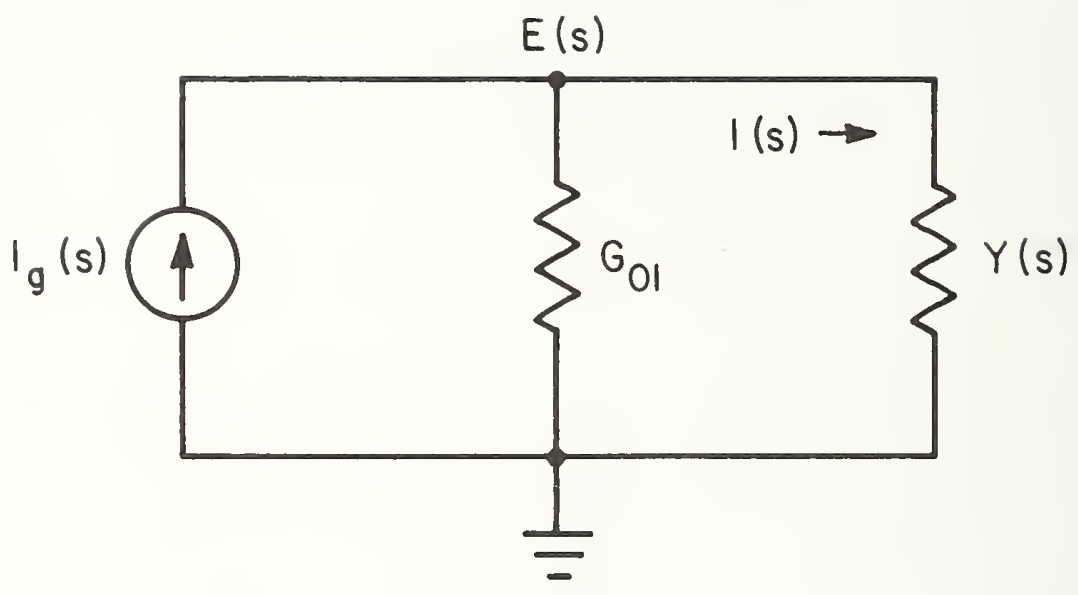

Figure A-5 The input equivalent circuit for a uniform lossless transmission line termination in an admittance $Y(s)$ and driven by a bandlimited generator $I_{g}(s), G_{01}$ is the transmission line characteristic admittance. 


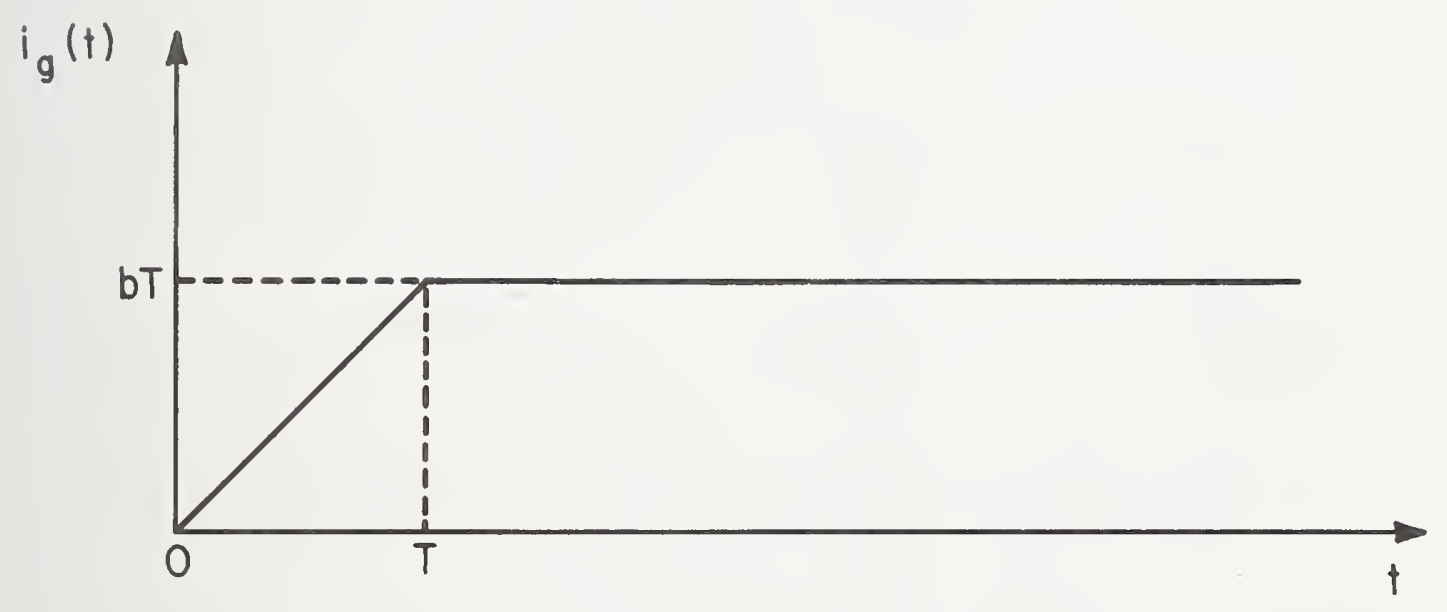

Figure A-6 The bandlimited driving current, $i_{g}(t)$.

83 


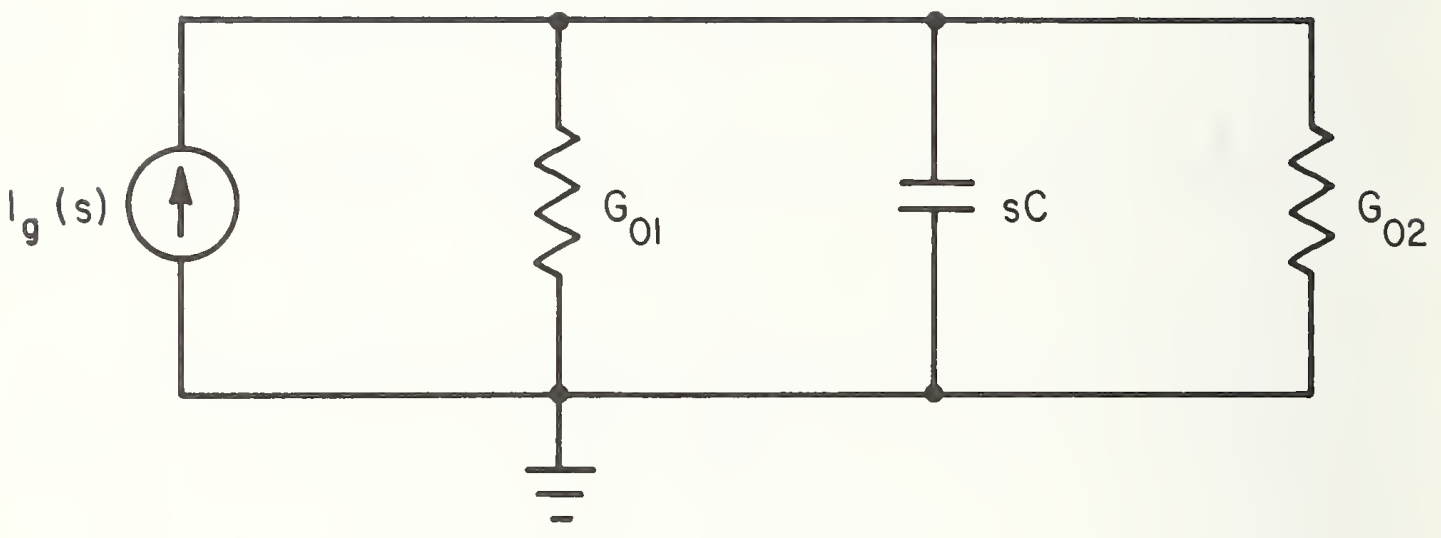

Figure A-7 The TDR equivalent circuit for a shunt capacitive discontinuity $\mathrm{s} C$ at the junction of two uniform transmission lines of characteristic admittances $\mathrm{G}_{01}$ and $\mathrm{G}_{02}$, respectively. 

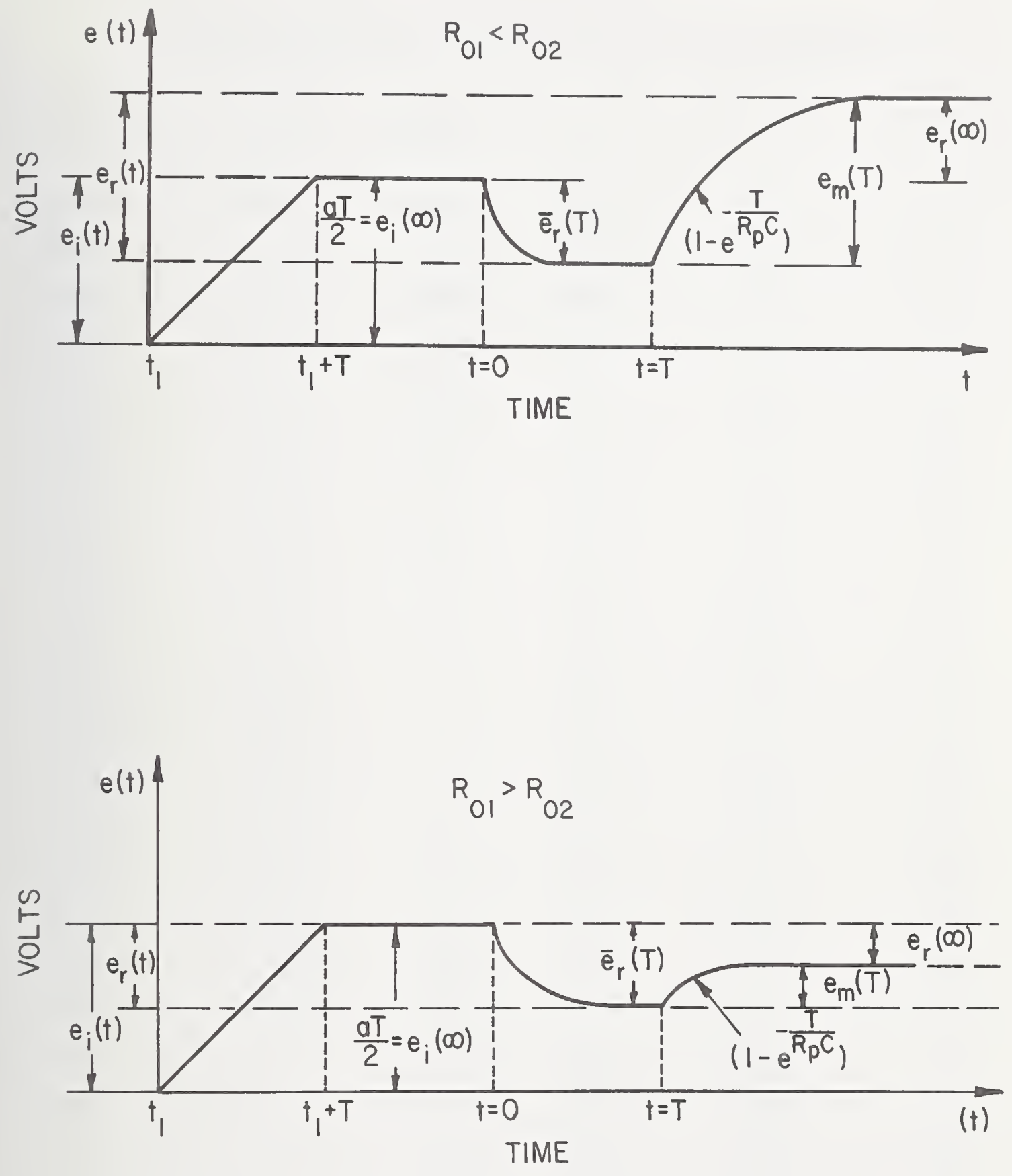

Figure A-8 Qualitative time domain responses for the total capacitive load voltage. 
APPENDIX A-2

WAVEFORM DATA FOR FIGURES 2.15 AND 2.16

$T(P S E C) \quad E A.(T)$

$\mathrm{EO}(\mathrm{T})$ T(PSEC.) EA(T)

$\mathrm{EO}(\mathrm{T})$

\begin{tabular}{|c|c|c|c|c|c|}
\hline 0 & .5000 & .5000 & -260 & .0329 & .0501 \\
\hline-10 & .4727 & .4487 & -270 & .0279 & .0449 \\
\hline-20 & .4455 & .4296 & -280 & .0235 & .0405 \\
\hline-30 & .4185 & .4044 & -290 & .0197 & .0366 \\
\hline-40 & .3917 & .3787 & -300 & .0164 & .0334 \\
\hline-50 & .3653 & .3547 & -310 & .0136 & .0301 \\
\hline-60 & .3395 & .3305 & -320 & .0113 & .0271 \\
\hline-70 & .3144 & .3072 & -330 & .0093 & .0249 \\
\hline-80 & .2901 & .2846 & -340 & .0076 & .0231 \\
\hline-90 & .2666 & .2623 & -350 & .0061 & .0210 \\
\hline-100 & .2444 & .2423 & -360 & .0051 & .0193 \\
\hline-110 & .2224 & .2245 & -370 & .0040 & .0176 \\
\hline-120 & .2020 & .2055 & -380 & .0032 & .0162 \\
\hline-130 & .1827 & .1866 & -390 & .0025 & .0149 \\
\hline-140 & .1645 & .1702 & -400 & .0020 & .0143 \\
\hline-150 & .1474 & .1551 & -410 & .0016 & .0137 \\
\hline-160 & .1317 & .1396 & -420 & .0012 & .0129 \\
\hline-170 & .1171 & .1265 & -430 & .0009 & .0118 \\
\hline-180 & .1035 & .1147 & -440 & & .0106 \\
\hline-190 & .0912 & .1035 & -450 & & .0095 \\
\hline-200 & .0800 & .0942 & -460 & & .0083 \\
\hline-210 & .0698 & .0849 & -470 & & .0073 \\
\hline-220 & .0606 & .0764 & -480 & & .0068 \\
\hline-230 & .0524 & .0693 & -490 & & .0062 \\
\hline-240 & .0451 & .0627 & -500 & & .0056 \\
\hline-250 & .0386 & .0563 & -510 & & .0050 \\
\hline
\end{tabular}


$\mathrm{T}(\mathrm{PSEC}$. ) EA(T) EO(T)

$-520 \quad .0044$

$-530 \quad .0039$

$-540 \quad .0033$

$-550 \quad .0027$

$-560 \quad .0021$

$-570 \quad .0016$

$-580 \quad .0013$

$-590 \quad .0010$ 
WAVEFORM DATA FOR FIGURES 2.15 AND 2.16

$\begin{array}{rrrrrr}\text { T(PSEC.) } & \text { EA (T) } & \text { EO }(\mathrm{T}) & \mathrm{T}(\mathrm{PSEC}) & \mathrm{EA}(\mathrm{T}) & \mathrm{EO}(\mathrm{T}) \\ 0 & .5000 & .5000 & 240 & .9354 & .9508 \\ 10 & .5272 & .5250 & 250 & .941 .9 & .9576 \\ 20 & .5541 & .5553 & 260 & .9478 & .9635 \\ 30 & .5808 & .5805 & 270 & .9527 & .9689 \\ 40 & .6069 & .6051 & 280 & .9573 & .9744 \\ 50 & .6324 & .6316 & 290 & .9613 & .9788 \\ 60 & .6573 & .6567 & 300 & .9649 & .9824 \\ 70 & .6814 & .6810 & 310 & .9679 & .9853 \\ 80 & .7047 & .7031 & 320 & .9707 & .9876 \\ 90 & .7269 & .7266 & 330 & .9731 & .9888 \\ 100 & .7482 & .7508 & 340 & .9752 & .9904 \\ 110 & .7685 & .7717 & 350 & .9770 & .9923 \\ 120 & .7877 & .7920 & 360 & .9787 & .9934 \\ 130 & .8057 & .8105 & 370 & .9800 & .9945 \\ 140 & .8227 & .8281 & 380 & .9812 & .9953 \\ 150 & .8386 & .8459 & 390 & .9823 & .9963 \\ 160 & .8534 & .8625 & 400 & .9832 & .9972 \\ 170 & .8670 & .8772 & 410 & .9840 & .9974 \\ 180 & .8796 & .8915 & 420 & .9847 & .9977 \\ 190 & .8912 & .9048 & 430 & .9853 & .9979 \\ 200 & .9019 & .9147 & 440 & .9859 & .9983 \\ 210 & .9115 & .9248 & 450 & .9863 & .9988 \\ 220 & .9203 & .9348 & 460 & .9867 & .9992 \\ 230 & .9282 & .9434 & 470 & .9871 & .9997\end{array}$


$\mathrm{T}(\mathrm{PSEC}.) \quad \mathrm{EA}(\mathrm{T}) \quad \mathrm{EO}(\mathrm{T}) \quad \mathrm{T}(\mathrm{PSEC}.) \quad \mathrm{EA}(\mathrm{T})$

$\mathrm{EO}(\mathrm{T})$

\begin{tabular}{|c|c|c|c|c|c|}
\hline 480 & .9874 & 1.0000 & 740 & .9913 & 1.0000 \\
\hline 490 & .9877 & 1.0000 & 750 & .9913 & \\
\hline 500 & .9880 & .998 & 760 & .9914 & \\
\hline 510 & .9882 & .9992 & 770 & .9915 & \\
\hline 520 & .9884 & .9986 & 780 & .9916 & \\
\hline 530 & .9886 & .9982 & 790 & .9917 & \\
\hline 540 & .9888 & .9972 & 800 & .9917 & \\
\hline 550 & .9890 & .9977 & 810 & .9918 & \\
\hline 560 & .9892 & .9975 & 820 & .9919 & \\
\hline 570 & .9893 & .9972 & 830 & .9920 & \\
\hline 580 & .9895 & .9971 & 840 & .9921 & \\
\hline 590 & .9896 & .9972 & 850 & .9922 & \\
\hline 600 & .9898 & .9974 & 860 & .9922 & \\
\hline 610 & .9899 & .9977 & 870 & .9922 & \\
\hline 620 & .9900 & .9979 & 880 & .9923 & \\
\hline 630 & .9901 & .9981 & 890 & .9924 & \\
\hline 640 & 9903 & .9985 & 900 & .9924 & \\
\hline 650 & .9904 & .9990 & 910 & .9925 & \\
\hline 660 & .9905 & .9996 & 920 & .9926 & \\
\hline 670 & .9906 & .9990 & 930 & .9926 & \\
\hline 680 & .9907 & 1.0000 & 940 & .9927 & \\
\hline 690 & .9908 & & 950 & .9927 & \\
\hline 700 & .9909 & & 960 & .9928 & \\
\hline 710 & .9910 & & 970 & .9929 & \\
\hline 720 & .9911 & & 980 & .9929 & \\
\hline 730 & .9912 & & 990 & .9930 & 1.0000 \\
\hline
\end{tabular}




\begin{tabular}{|c|c|c|c|c|c|}
\hline $\mathrm{T}(\mathrm{PSEC})$. & $\mathrm{EA}(\mathrm{T})$ & $\mathrm{EO}(\mathrm{T})$ & $\mathrm{T}$ (PSEC.) & $\mathrm{EA}(\mathrm{T})$ & $\mathrm{EO}(\mathrm{T})$ \\
\hline 1000 & .9930 & 1.0000 & 1260 & .9942 & 1.0000 \\
\hline 1010 & .9931 & & 1270 & .9942 & \\
\hline 1020 & .9931 & & 1280 & .9943 & \\
\hline 1030 & .9932 & & 1290 & .9943 & \\
\hline 1040 & .9932 & & 1300 & .9943 & \\
\hline 1050 & .9933 & & 1310 & .9944 & \\
\hline 1060 & .9933 & & 1320 & .9944 & \\
\hline 1070 & .9934 & & 1330 & .9944 & \\
\hline 1080 & .9934 & & 1340 & .9945 & \\
\hline 1090 & .9935 & & 1350 & .9945 & \\
\hline 1100 & .9935 & & 1360 & .9945 & \\
\hline 1110 & .9936 & & 1370 & .9946 & \\
\hline 1120 & .9936 & & 1380 & .9946 & \\
\hline 1130 & .9937 & & 1390 & .9946 & \\
\hline 1140 & .9937 & & 1400 & .9947 & \\
\hline 1150 & .9937 & & 1410 & .9947 & \\
\hline 1160 & .9938 & & 1420 & .9947 & \\
\hline 1170 & .9938 & & 1430 & .9948 & \\
\hline 1180 & .9939 & & 1440 & .9948 & \\
\hline 1190 & .9939 & & 1450 & .9948 & \\
\hline 1200 & .9940 & & 1460 & .9949 & \\
\hline 1210 & .9940 & & 1470 & .9949 & \\
\hline 1220 & .9940 & & 1480 & .9949 & \\
\hline 1230 & .9941 & & 1490 & .9949 & \\
\hline 1240 & .9941 & & 1500 & .9950 & \\
\hline 1250 & .9941 & 1.0000 & 1510 & .9950 & 1.0000 \\
\hline
\end{tabular}


T(PSEC.) EA(T) EO(T) T(PSEC. ) EA(T)

$\mathrm{EO}(\mathrm{T})$

\begin{tabular}{|c|c|c|c|c|c|}
\hline 1520 & .9950 & 1.0000 & 1780 & .9957 & 1.0000 \\
\hline 1530 & .9950 & & 1790 & .9957 & \\
\hline 1540 & .9951 & & 1800 & .9957 & \\
\hline 1550 & .9951 & & 1810 & .9957 & \\
\hline 1560 & .9951 & & 1820 & .9958 & \\
\hline 1570 & .9951 & & 1830 & .9958 & \\
\hline 1580 & .9952 & & 1840 & .9958 & \\
\hline 1590 & .9952 & & 1850 & .9958 & \\
\hline 1600 & .9952 & & 1860 & .9958 & \\
\hline 1610 & .9952 & & 1870 & .9959 & \\
\hline 1620 & .9953 & & 1880 & .9959 & \\
\hline 1630 & .9953 & & 1890 & .9959 & \\
\hline 1640 & .9953 & & 1900 & .9959 & \\
\hline 1650 & .9954 & & 1910 & .9959 & \\
\hline 1660 & .9954 & & 1920 & .9960 & \\
\hline 1670 & .9954 & & 1930 & .9960 & \\
\hline 1680 & .9954 & & 1940 & .9960 & \\
\hline 1690 & .9955 & & 1950 & .9960 & \\
\hline 1700 & .9955 & & 1960 & .9960 & \\
\hline 1710 & .9955 & & 1970 & .9961 & \\
\hline 1720 & .9955 & & 1980 & .9961 & \\
\hline 1730 & .9956 & & 1990 & .9961 & 1 \\
\hline 1740 & .9956 & & 2000 & .9961 & 1.0000 \\
\hline 1750 & .9956 & & & & \\
\hline 1760 & .9956 & & & & \\
\hline 1770 & .9956 & 1.0000 & & & \\
\hline
\end{tabular}




\begin{tabular}{|c|c|c|}
\hline $\begin{array}{l}\text { U.C. DEPT. OF COMM. } \\
\text { BIBLIOGRAPHIC DATA } \\
\text { SHEET }\end{array}$ & $\begin{array}{l}\text { 1. PUBLICATION OR REPORT NO. } \\
\text { NBSIR } 73-304\end{array}$ & 3. Recipient's Accession No. \\
\hline \multirow{2}{*}{\multicolumn{2}{|c|}{$\begin{array}{l}\text { 4. TITLE AND SUBTITLE } \\
\text { REFERENCE-WAVEFORM GENERATION USING DEBYE } \\
\text { DIELECTRIC DISPERSION }\end{array}$}} & $\begin{array}{l}\text { 5. Publication Date } \\
\text { Dec ember } 1972\end{array}$ \\
\hline & & 6. Performing Organization Code \\
\hline 7. AUTHOR(s) N.S. N & nan, R. M. Jickling and D. R. Holt & 8. Performing Organization \\
\hline \multicolumn{2}{|c|}{ 9. PERFORMING ORGANIZATION NAME AND ADDRESS } & $\begin{array}{l}\text { 10. Project/Task/Work Unit No. } \\
2725390\end{array}$ \\
\hline \multicolumn{2}{|c|}{$\begin{array}{l}\text { NATIONAL BUREAU OF STANDARDS } \\
\text { DEPARTMENT OF COMMERCE } \\
\text { Boulder, Colorado } 80302\end{array}$} & 11. Contract/Grant No. \\
\hline \multirow{2}{*}{\multicolumn{2}{|c|}{$\begin{array}{l}\text { 12. Sponsoring Organization Name and Address } \\
\text { Department of Defense } \\
\text { Calibration Coordination Group } 69-33 \\
\text { U.S. Army Metrology \& Calibration Center } \\
\text { Redstone Arsenal, Alabama } 35809\end{array}$}} & $\begin{array}{l}\text { 13. Type of Report \& Period } \\
\text { Covered } \\
\text { Final }\end{array}$ \\
\hline & & 14. Sponsoring Agency Code \\
\hline
\end{tabular}

15. SUPPLEMENTARY NOTES

16. ABSTRACT (A 200-word or Iess factual summary of most significant information. If document includes a significant bibliography or literature survey, mention it here.)

This report discusses the theory, construction, and operation of ReferenceWaveform Generators using a tunnel diode transition-waveform generator driving 4.65 meter (15 foot), 3. 10 meter (10 foot), and 1.55 meter ( 5 foot) liquid-dielectric uniform lossy coaxial lines to produce known 0.2 volt transition waveforms across 50 ohms with (10-90\%) transition times from 205 to 560 picosecond. Each resultant Available-Waveform is characterized in terms of its departure from the step response of the uniform lossy coaxial line operating into a 50 ohm load. The liquid dielectric solutions are dispersive with relaxation times of the order of 4 picoseconds in which heptane is the solvent and the ketones, butanone, heptanone and octanone are the solutes in concentrations ranging from $0.25-$ to 2-molal.

17. KEY WORDS (Alphabetical order, separated by semicolons) Pulse distortion in transmission lines; pulse techniques; reference waveform generation; time domain measurements; transient response Debye dielectric.

18. AVAILABILITY STATEMENT

[X] UNLIMIT ED.

FOR OFFICI AL DISTRIBUTION. DO NOT RELEASE TO NTIS.
19. SECURITY CLASS (THIS REPURT)

UNCL ASSIF IED

20. SECURITY CLASS (THIS PAGE)

UNCL ASSIFIED
21. NO. OF PAGES

22. Price 